Argonne

\title{
Impact of High Penetration Distributed Energy Resources on the Bulk Electric System
}

\author{
Energy Systems Division
}




\begin{abstract}
About Argonne National Laboratory
Argonne is a U.S. Department of Energy laboratory managed by UChicago Argonne, LLC under contract DE-AC02-06CH11357. The Laboratory's main facility is outside Chicago, at 9700 South Cass Avenue, Lemont, Illinois 60439. For information about Argonne and its pioneering science and technology programs, see www.anl.gov.
\end{abstract}

\title{
DOCUMENT AVAILABILITY
}

Online Access: U.S. Department of Energy (DOE) reports produced after 1991 and a growing number of pre-1991 documents are available free at OSTI.GOV (http://www.osti.gov/),

a service of the US Dept. of Energy's Office of Scientific and Technical Information.

Reports not in digital format may be purchased by the public from the National Technical Information Service (NTIS):

U.S. Department of Commerce

National Technical Information Service

5301 Shawnee Road

Alexandria, VA 22312

www.ntis.gov

Phone: (800) 553-NTIS (6847) or (703) 605-6000

Fax: (703) 605-6900

Email: orders@ntis.gov

Reports not in digital format are available to DOE and DOE contractors from the Office of Scientific and Technical Information (OSTI):

U.S. Department of Energy

Office of Scientific and Technical Information

P.O. Box 62

Oak Ridge, TN 37831-0062

www.osti.gov

Phone: (865) 576-8401

Fax: (865) 576-5728

Email: reports@osti.gov

\section{Disclaimer}

This report was prepared as an account of work sponsored by an agency of the United States Government. Neither the United States Government nor any agency thereof, nor UChicago Argonne, LLC, nor any of their employees or officers, makes any warranty, express or implied, or assumes any legal liability or responsibility for the accuracy, completeness, or usefulness of any information, apparatus, product, or process disclosed, or represents that its use would not infringe privately owned rights. Reference herein to any specific commercial product, process, or service by trade name, trademark, manufacturer, or otherwise, does not necessarily constitute or imply its endorsement, recommendation, or favoring by the United States Government or any agency thereof. The views and opinions of document authors expressed herein do not necessarily state or reflect those of the United States Government or any agency thereof, Argonne National Laboratory, or UChicago Argonne, LLC. 
ANL/ESD-20/3

\section{Impact of High Penetration Distributed Energy Resources on the Bulk Electric System}

by

Ning Kang', Rojan Bhattarai', James T. Reilly ${ }^{2}$, and Sara Ahmed ${ }^{3}$

'Energy Systems Division, Argonne National Laboratory

(authors are currently affiliated with Idaho National Laboratory)

${ }^{2}$ Reilly Associates

3University of Texas at San Antonio

for

Office of Electricity, U.S. Department of Energy

May 2021 
This page intentionally blank. 


\section{CONTENTS}

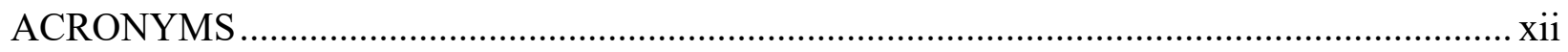

ACKNOWLEDGMENTS .........................................................................................

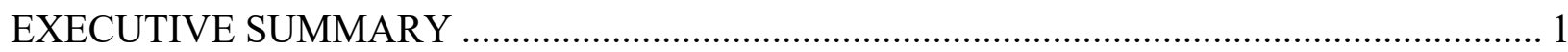

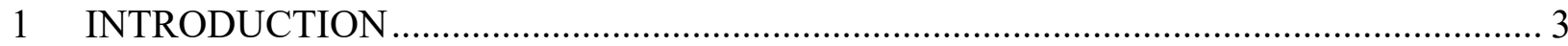

2 MODELING APPROACHES TO STUDY THE IMPACT OF DER ON BES ................... 5

2.1 Distribution System Modeling Approaches for BES Impact Studies ......................... 5

2.1.1 Description of Test System ................................................................ 5

2.1.2 Full Modeling of Distribution Systems ................................................... 7

2.1.3 PV System Modeling in Full Distribution System Models ............................ 8

2.1.4 Aggregated Modeling of Distribution Systems ............................................. 10

2.1.5 Aggregated Distributed PV Model (PVD1 Model).................................... 12

2.2 Performance Comparison of Two Distribution System Modeling Approaches

in BES Impact Studies ........................................................................................ 14

2.2.1 Performance Comparison of Two Modeling Approaches though a Load Step Change Case Study ................................................................... 15

2.2.2 Performance Comparison of Two Modeling Approaches through a Transmission System Fault Case Study ................................................. 17

3 DERS IMPACT ON AND CONTROL STRATEGIES TO IMPROVE

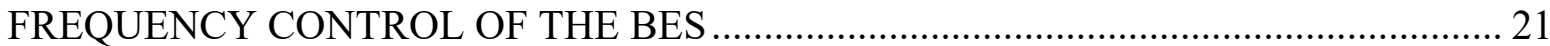

3.1 Control Strategies for Enhancing BES Frequency Response .................................. 21

3.1.1 Fast Frequency Regulation for BES Contingencies .................................. 21

3.1.2 Proposed VSG-based Control for DERs .................................................. 22

3.1.3 Impact of DERs with and without VSG on BES .................................... 25

3.2 DERs' Impact on BES Net Load Variability and Ramping and Mitigation

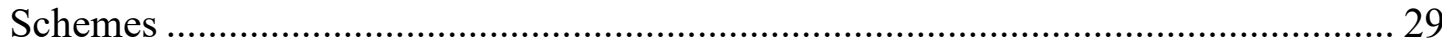

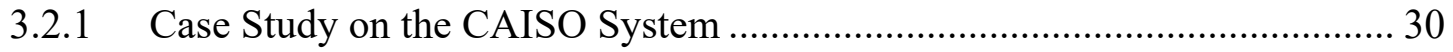

3.2.2 Case Study on Kundur Two-Area System................................................ 33

4 DERs' IMPACT ON AND CONTROL STRATEGIES TO IMPROVE THE VOLTAGE STABILITY OF THE BES …................................................................. 37

4.1 Impact of DER VVC on BES Voltage During Normal Operations.......................... 37

4.1.1 Comparison of Performance of Line Regulators and Reactive Power Support from DERs on Voltage Regulation of the BES ........................... 37

4.1.2 Impact of Different DER Volt-VAR Curves (under Same Time Delay) on BES Voltage Regulation. 
4.1.3 Impact of Different Time Delays of DERs' Volt-VAR Control on BES Voltage Regulation...................................................................... 48

4.1.4 Impact of Interaction between Multiple DERs with VVC Enabled on BES

4.2 Effect of DER Reactive Power Support Capability to Enhance BES Voltage

Control During Fault Conditions ....................................................................... 57

4.2.1 Impact of DER without Reactive Power Support on BES Stability .............. 57

4.2.2 Effect of DERs with Reactive Power Support to Enhance BES Stability..... 60

5 IMPACT OF DER PENETRATION ON DISTRIBUTION SYSTEM

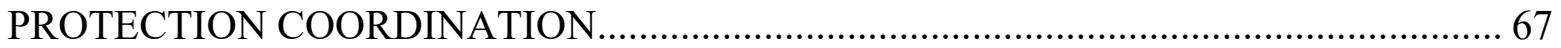

5.1 Description of the Distribution System for Protection Studies................................. 68

5.2 Increased Fault Current............................................................................. 70

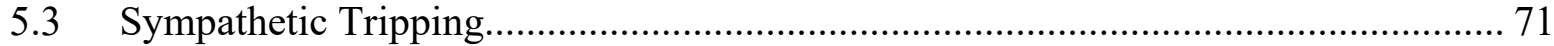

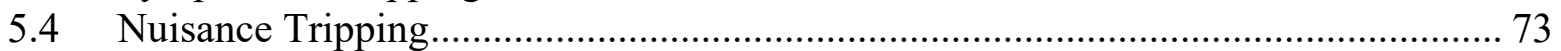

5.5 Desensitizing Feeder Head Relays..................................................................... 74

5.6 Potential Solutions for Distribution System Protection ......................................... 76

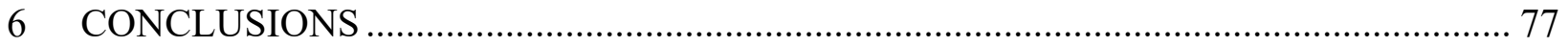

APPENDIX A: PROCEDURE TO OBTAIN AGGREGATED DISTRIBUTION

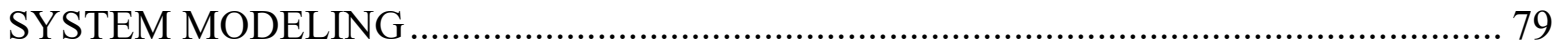

APPENDIX B: OPERATING CONDITIONS FOR THE T\&D NETWORKS FOR THE

CASE STUDIES $(16 \%, 32 \%$, AND 48\% WITH RESPECT TO LOAD IN AREA 2) ....... 85

REFERENCES 89

\section{FIGURES}

Figure 1 Schematic Diagram of the Modified Kundur Two-Area System.............................. 7

Figure 2 Schematic Diagram of the Test Distribution Feeder. .............................................. 7

Figure 3 Schematic Diagram of a Transmission System with Partial Load Replaced by

Distribution Feeders Integrated with DERs. ........................................................... 8

Figure 4 Schematic Diagram of the Inverter-Interfaced DER Model [5] .............................. 10

Figure 5 Aggregated Steady-State (a) and Dynamic (b) Model of the Distribution System Integrated with Distributed PV Systems. ................................................................... 11

Figure 6 Schematic Diagram of PVD1 [7] ....................................................................... 13 
Figure 7 System Frequency Deviation from a Load Increase of 100 MW for Aggregated and Full Distribution System Models................................................................................... 15

Figure 8 Substation Power Flow Composition for Aggregated and Full Distribution System Models

Figure 9 PV System PCC Voltage Deviation Comparison with Aggregated and Full Distribution System Models. 16

Figure 10 PCC Frequency Comparison for Distributed PV Systems in the Full Model and for the PVD1 in the Aggregated Model.

Figure 11 System Frequency Deviation Following a Self-Clearing, Three-Phase Bolted Fault at the Middle of the Tie-Line for Aggregated and Full Distribution System Models. 18

Figure 12 Substation Power Flow Composition for Aggregated and Full Distribution System Models. 18

Figure 13 PCC Frequency Comparison for Distributed PV Systems in Full Model and PVD1 in Aggregated Model 19

Figure 14 PV Output Comparison for Aggregated and Full Distribution System Models.......... 19

Figure 15 PV PCC Voltage for the Distributed PV Model and PVD1 Model. 20

Figure 16 Represenation of a Multiple Time-frame Frequency Response in a Power

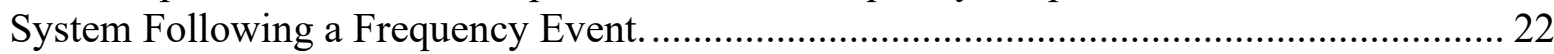

Figure 17 The Proposed VSG-based Multi-Loop Control Method in a PV DG. ......................... 23

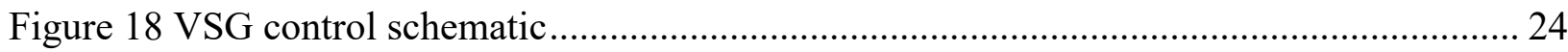

Figure 19 T\&D Combined System with VSG Technologies........................................................... 26

Figure 20 System Frequency with Different Inertia in SGs. ..................................................... 27

Figure 21 System Frequency with No DERs, with DERs but No Inertia Emulation, and with DERs and Inertia Emulation, respectively, at $200 \mathrm{MW}$ 28

Figure 22 System Frequency with No DERs, with DERs but No Inertia Emulation, and with DERs and Inertia Emulation, respectively, at $400 \mathrm{MW}$ 28

Figure 23 Effect of DER with VSG on BES Frequency 29

Figure 24 Average Net Load in the CAISO Studies (in red) and the Actual Load (black) in the System. 
Figure 25 PV Power Generation in the CAISO Area on May 1, 2018.

Figure 26 Net Load in the CAISO Studies with and without PVs and with the Use of the BESS. 32

Figure 27 Net Load as Seen on the Kundur Two-Area System with and without PVs. 34

Figure 28 Comparison of the Net Load Profile with no PV, with PV and no BESS, and with both PV and BESS.

Figure 29 Flowchart of the Implemented Algorithm for Minimizing the Net Load Ramping Rate in the BES. 35

Figure 30 DER Volt-VAR Curve Illustration. 38

Figure 31 Transmission Bus Voltage Comparison (note: LREG = line regulation; VVAR $=$ Volt-VAR ). 39

Figure 32 Distribution System Substation Power Flow Comparison. 40

Figure 33 Tie-line Active Power Flow Comparison. 41

Figure 34 PV Reactive Power Output Comparison. 42

Figure 35 PV Reactive Power Output Comparison for Different Volt-VAR Settings............... 44

Figure 36 PV PCC Voltage Comparison for Different Volt-VAR Settings. ............................ 45

Figure 37 Tie-Line Bus Voltage Comparison for Different Volt-VAR Settings....................... 45

Figure 38 T\&D Boundary Bus Active Power Flow Comparison for Different Volt-VAR Settings. 46

Figure 39 T\&D Boundary Bus Reactive Power Flow Comparison for Different VoltVAR Settings. 46

Figure 40 Tie-Line Power Flow Comparison for the Case with Different Volt-VAR Settings. 47

Figure 41 Generator Rotor Speed Comparison for Different Volt-VAR Settings. 48

Figure 42 PV Reactive Power Output Comparison for the Scenarios with and without Time Delays in Volt-VAR Control.

Figure 43 PV PCC Voltage Comparison for the Scenarios with and without Time Delays in Volt-VAR Control. 50

Figure 44 Tie-Line Bus Voltage Comparison for the Scenarios with and without Time Delays in Volt-VAR Control. 50 
Figure 45 T\&D Boundary Bus Power Flow Comparison for the Scenarios with and without Time Delays in Volt-VAR Control.

Figure 46 Tie-Line Power Flow Comparison for the Scenarios with and without Time Delays in Volt-VAR Control............................................................................. 52

Figure 47 Generator Rotor Speed Comparison for the Scenarios with and without Time Delays in Volt-VAR Control. 52

Figure 48 PV Reactive Power Output Comparison. .............................................................. 54

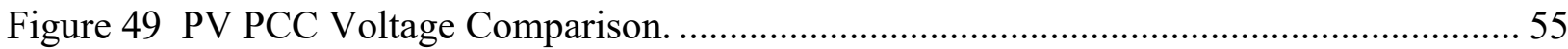

Figure 50 Tie-Line Bus Voltage Comparison................................................................. 56

Figure 51 T\&D Boundary Bus Active and Reactive Power Flow Comparison........................ 57

Figure 52 Comparison of Tie-Line Active Power Flow......................................................... 58

Figure 53 Comparison between Tie-Line Sending-End and Receiving-End Voltages. ............. 59

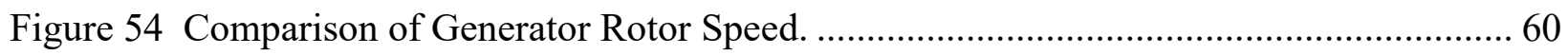

Figure 55 Comparison of Tie-Line Active Power Flow........................................................ 61

Figure 56 Comparison between Tie-Lines' Sending-End and Receiving-End Voltages. ........... 62

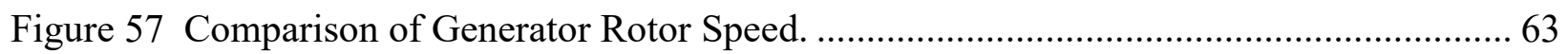

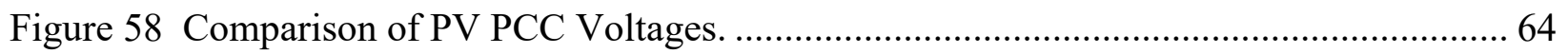

Figure 59 Comparison of PV PCC Reactive Power Output. .............................................. 65

Figure 60 Comparison of PV PCC Active Power Output. ..................................................... 66

Figure 61 Schematic Diagram of the Distribution System for Protection Studies. ................... 69

Figure 62 Inverse Time Current Characteristics of the Recloser and Fuses Used in the

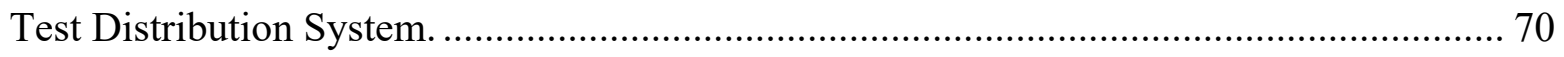

Figure 63 Illustration of Sympathetic Tripping. ......................................................... 71

Figure 64 Feeder \#1 Recloser Status Comparison for a Case with and without DG................. 73

Figure 65 Desensitizing of a Feeder Head Protection Relay due to DG Integration.................. 74

Figure 66 Impact of DGs on the Recloser's Operation Time. ................................................. 76 
Figure A-1 (a) Equivalent Distribution System with Individual Aggregated Distribution Feeders; and (b) Equivalent Aggregated Distribution System. 82

Figure A-2 Flowchart of the Process for Obtaining the Aggregated Model of a Distribution System. 84

\section{TABLES}

Table 1 Original Kundur Two-Area System Parameters.

Table 2 DER Interconnection System Response to Abnormal Voltages as per IEEE 1547a-2014.

Table 3 DER Interconnection System Response to Abnormal Frequencies as per

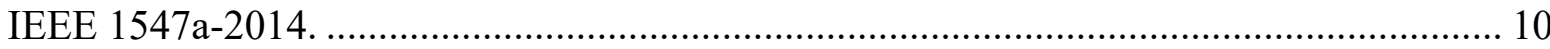

Table 4 PVD1 Parameters Used in the Case Studies.................................................................... 13

Table 5 Operating Conditions for the T\&D Networks for the Case Studies............................... 14

Table 6 Comparison of Net Load Ramping Rates at Different PV Power Output Levels. ......... 33

Table 7 Combination of Various Availabilities of DER VVC and Line Regulators.................... 37

Table 8 Operational Settings Used for Line Regulators............................................................ 38

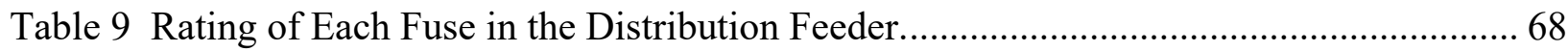

Table 10 Fault Current Distribution with Varying PV Penetration Levels. ................................. 71

Table 11 Summary of Feeder Head Fault Currents with Varying Levels of DG

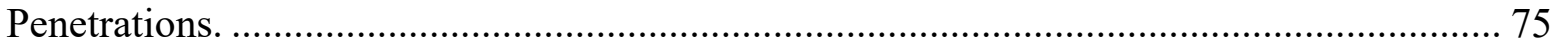

Table 12 Estimated Equivalent Feeder Parameters for a Single Distribution Feeder.................... 81

Table B-1 Operating Conditions for the T\&D Networks for the Case Studies with a 16\%

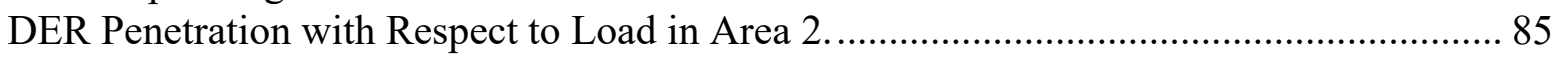

Table B-2 Operating Conditions for the T\&D Networks for the Case Studies with a 32\% DER Penetration with Respect to Load in Area 2............................................................. 86

Table B-3 Operating Conditions for the T\&D Networks for the Case Studies with a 48\% Penetration with Respect to Area 2. 
This page intentionally blank. 


\section{ACRONYMS}

\begin{tabular}{|c|c|}
\hline $\mathrm{AC}$ & alternating current \\
\hline AGC & automatic generation control \\
\hline BES & bulk electric system \\
\hline BESS & battery energy storage systems \\
\hline CAISO & California Independent System Operator \\
\hline DER & distributed energy resource \\
\hline DC & direct current \\
\hline DG & distributed generator \\
\hline EPS & electric power system \\
\hline IEEE & Institute of Electrical and Electronics Engineers \\
\hline MPP & maximum power point \\
\hline OF & over-frequency \\
\hline PCC & point of common coupling \\
\hline PLL & phase lock loop \\
\hline PQ & active $(\mathrm{P})$ and reactive $(\mathrm{Q})$ power \\
\hline PV & photovoltaic \\
\hline PSS & power system stabilizer \\
\hline SG & synchronous generator \\
\hline T\&D & transmission and distribution \\
\hline UF & under-frequency \\
\hline VSG & virtual synchronous generator \\
\hline VVAR & Volt-Var \\
\hline VVC & Volt-VAR control \\
\hline WECC & Western Electricity Coordinating Council \\
\hline
\end{tabular}




\section{ACKNOWLEDGMENTS}

This report was prepared by UChicago Argonne, LLC, operator of Argonne National Laboratory. Argonne's work was supported by the U.S. Department of Energy (DOE) under contract DE-AC02-06CH11357.

The authors wish to acknowledge the sponsorship and guidance provided by Ali Ghassemian of DOE's Office of Electricity. 


\section{EXECUTIVE SUMMARY}

Both the growth of distributed energy resources (DERs) and the retirement of central generation (mostly synchronized conventional power plants using fossil-fuel-based resources) have implications for the steady-state and dynamic performance of the bulk electric system (BES). Specifically, the high penetration of DERs in the BES has an impact on its stability and reliability, as well as on the distribution network to which the DERs are directly connected. The growth of DERs - supported by lower costs of renewable energy and natural gas, advances in inverter technology, and favorable federal and state government policies - means that this impact is becoming more significant. This report presents the results of analysis on how the high levels of DERs integration may affect the BES.

To enable these analyses, this report first introduces two approaches to modeling the distribution system and interconnected DERs: (1) an aggregated modeling approach, and (2) a full modeling approach. The aggregated distribution system model comprises an equivalent/aggregate distribution system model (including an aggregate load model and an equivalent feeder segment) and an aggregate dynamic DER model. The aggregated distribution system model replaces the original load centers in a transmission system model to enable studying the impact of DERs on the BES. In the full distribution system modeling approach, on the other hand, the non-aggregated distribution system and individual DERs are modeled. Combining the full distribution system model with a transmission system model is an alternate approach to studying the impact of DERs on the BES. The transmission system and the full distribution system modeling as a whole is referred to as the "T\&D combined model." The performance of both distribution system modeling approaches is compared and contrasted in BES stability studies. In this work, a T\&D combined model is developed in MATLAB/Simulink software. The T\&D combined model features the full distribution system models of the IEEE 34-node feeder and the transmission system model of the Kundur Two-Area system.

The impacts of the high penetration of DERs on the BES are analyzed in terms of BES frequency and voltage regulation. On the distribution side, the impact of high penetration of DERs on the distribution system's protection coordination is assessed. Certain control and management strategies that may utilize advanced controls of DERs (e.g., virtual inertia, voltageor Volt-VAR control [VVC]) are used to demonstrate their effectiveness in helping maintain BES frequency and voltage stability.

Key findings from case studies performed using the T\&D combined model are summarized as follows:

- Compared to a base case without any DERs, when DERs without virtual inertia are integrated, the system frequency nadir decreases following a sudden load increase. When DERs equipped with virtual inertia are present, the system frequency nadir increases following a sudden load increase event as compared to the base case without any DERs. 
- When photovoltaic (PV) penetration increases, net load variability and ramping challenges arise in the BES, as well as in the distribution network. A substation-based smoothing algorithm that utilizes a battery energy storage system is effective in flattening the BES net load.

- Various slopes of DERs' VVC curve coupled with time delays can affect BES voltage regulations during normal operations. A slow response coupled with steep Volt-VAR settings in DERs may cause system instability. It is crucial that the DER voltage support settings be well coordinated with their response lags.

- Without local reactive power support, increased DER penetration results in oscillations and sags in voltage of the transmission system's power flow and aggravates frequency recovery. Although DERs' dynamic reactive power support can help maintain system stability overall, the system is sensitive to the DER settings with respect to reactive power support.

- High DER penetration in a distribution network may increase fault current, cause sympathetic tripping and nuisance tripping, and desensitize feeder head protection relays.

Overall, the results of these case studies suggest that increasing the integration of DERs can have detrimental as well as positive impacts on the BES. Further studies with large-scale systems, using an enhanced T\&D combined model and recognizing the latest operational practices for DER management, are needed to build on the findings of this report and enhance the understanding of the ways that DERs affect the BES. 


\section{INTRODUCTION}

The growth of distributed energy resources (DERs), mostly generation using intermittent renewable energy sources, along with the retirement of central generation (mostly conventional power plants using fossil-fuel-based resources) has implications for the steady-state and dynamic performance of the BES. Among the DERs connected to the distribution system, photovoltaic (PV) systems are the most prevalent and have been installed at an increasing rate. Until recently, the penetration levels of DERs have not been high enough to create significant impacts on the reliability and security of power system operation. However, in recent years, the penetration levels of DERs have risen to a level that their impacts on the bulk electric system (BES) should be considered in a detailed fashion in planning and operations.

The PV and wind systems connected to the transmission network have been well studied and represented in BES stability studies. However, because of the distributed nature of DERs, especially PV systems, and the variability of their support capabilities depending on vendors, manufactured years, and costs, modeling distributed PV systems to accurately capture their impact on the BES remains challenging.

Chapter 2 explores the modeling approaches for distribution systems integrated with DERs to study the impact of DERs on the BES, namely, the aggregated modeling approach and the full modeling approach. The aggregated distribution system model comprises an equivalent/aggregate distribution system model (including an aggregate load model and an equivalent feeder segment) and an aggregate dynamic DER model. The aggregated distribution system model is connected to a transmission system model to enable studying the impact of DERs on the BES. In the full distribution system modeling approach, on the other hand, the nonaggregated distribution system and individual DERs are modeled. Connecting the full distribution system model to a transmission system model on the same simulation platform offers another approach to studying the impact of DERs on the BES. The transmission system and the full distribution system modeling as a whole are referred to as the T\&D combined model. The performance of both distribution system modeling approaches is compared and contrasted in BES stability studies in this chapter. While the aggregated modeling approach provides a simplified approach to investigate the impact of DERs on the BES, the full modeling approach is more appropriate to account for the full spectrum of DER dynamics, including phase lock loop (PLL) dynamics, direct current (DC) link dynamics, and time dependency of protection elements, as well as various legacy components of distribution systems such as line regulators and switched capacitors.

When investigating DERs' impact on the BES, one should consider that modern-day DERs are capable of providing both active and reactive power to the system through the use of advanced inverter controls. As a matter of fact, recent DER interconnection standards such as Institute of Electrical and Electronics Engineers (IEEE) Standard 1547-2018 now require DERs to be able to provide frequency and voltage support to the power system. These ancillary services can now be utilized to support the overall system reliability subject to the approval of the area electric power system (EPS) operator. In Chapter 3, DERs' impact on and the control strategies to improve the frequency control of the BES are investigated. Specifically, a virtual- 
synchronous-generator-based control scheme is proposed for DERs, and its contribution to the BES inertial response is demonstrated. Moreover, DERs' impact on BES net load variability and ramping are studied, and mitigation schemes are discussed and implemented. This chapter also highlights the effectiveness of the mitigation schemes in smoothing the BES net load.

Chapter 4 addresses DERs' impact on and the control strategies to improve the voltage stability of the BES. Using the T\&D combined model, this chapter first explores how the combination of various slopes and time delays of DER VVC curves can affect BES voltage control during normal operations. The impact of interactions between multiple DERs with VVC enabled on the BES is also examined through case studies. This chapter then expands beyond considering normal operations by presenting case studies that demonstrate that the dynamic DER reactive power support can help maintain BES voltages during abnormal conditions.

The conventional distribution system's protection scheme faces challenges with the high penetration of DERs. Although the contribution of fault current from each DER is limited, the high penetration of DERs can wind up contributing a considerable amount of fault current. These sources can change the fault current distribution and magnitude and cause new problems for the operation of existing overcurrent protection schemes. Chapter 5 demonstrates some of the emerging protection issues that arise because of high DER penetration including increased fault current, sympathetic tripping, nuisance tripping, and desensitizing feeder head protection relays.

Chapter 6 concludes the report with a summary of the work conducted, insights and observations made in these studies, and mitigation solutions for promoting secure and reliable BES operations. 


\section{MODELING APPROACHES TO STUDY THE IMPACT OF DER ON BES}

This chapter comprises two sections. The first introduces two approaches to modeling the distribution system and interconnected DERs for BES impact studies. The second section compares the performance of both distribution system modeling approaches through various case studies.

\subsection{DISTRIBUTION SYSTEM MODELING APPROACHES FOR BES IMPACT STUDIES}

Conventionally, for system planning and operational studies, the BES has been modeled by a mesh network connecting equivalent generators and static passive load. The equivalent generators are representatives of central power plants, and the static passive loads are representatives of the distribution system. Future grids with high penetration of DERs are expected to significantly affect the power flows between the transmission network and the distribution system. In these scenarios, modeling the distribution system integrated with DERs is recommended for both BES steady-state and dynamic studies. Modeling a single static load for distribution systems with a high penetration of DERs is insufficient as this model fails to represent the DERs' dynamic characteristics and the effects of the unbalanced system. Although modeling the distribution system and the multiple DERs connected at various nodes in the distribution system as thoroughly as possible is desirable, the computational burden and complexity of such a model may limit the types of studies performed. Therefore, certain simplifications may be achieved by aggregating models with similar characteristics and/or using reduced-order dynamic equivalent models [1].

In this report, two approaches in modeling the distribution system and its integrated DERs are introduced to study the impact of DERs on the BES, namely, an aggregated modeling approach and a full modeling approach. The aggregated distribution system model comprises an equivalent/aggregate distribution system model (including a substation transformer, an aggregate load model, and an equivalent feeder segment) and an aggregate static/dynamic DER model. The aggregated distribution system model is connected to a transmission system model to enable studying the impact of DERs on the BES. In the full distribution system modeling approach, on the other hand, the non-aggregated distribution system and individual DERs are modeled. Connecting the full distribution system model to a transmission system model on the same simulation platform offers another approach to studying the impact of DERs on the BES. The transmission system and the full distribution system modeling as a whole are referred to as the T\&D combined model.

\subsubsection{Description of Test System}

The distribution system modeled in both approaches is integrated into the transmission system model. Figure 1 shows the schematic diagram of the modified Kundur's Two-Area system [2], which represents the BES model used for an impact study of DERs on the BES. This 
test system has been developed on the MATLAB/Simulink platform. The original Kundur TwoArea system parameters are shown in Table 1. Compared to the original BES system in [2], the load connected at bus \#9 in the BES model is partitioned to represent a distribution system. The feeder modeled in the distribution system is shown in Figure 2, which is the modified IEEE 34-node test feeder [3]. As Figure 2 shows, the distribution feeder consists of loads of different types and has distributed PV systems connected at various nodes within it.

Table 1 Original Kundur Two-Area System Parameters.

\begin{tabular}{lcccc}
\hline \multicolumn{1}{c}{ Generator Data } & G1 & G2 & G3 & G4 \\
\hline Generator Capacity (MVA) & 900 & 900 & 900 & 900 \\
Power Output (MW) & 700 & 700 & 719 & 700 \\
Inertia (s) & 6.5 & 6.5 & 6.715 & 6.715 \\
& & & \\
Load Data & & & \\
Load in Area 1 & & & \\
Load in Area 2 & 1767 MW, 100 MVAR (inductive), and 387 MVAR (capacitive) \\
\hline
\end{tabular}

Please note that in the developed modified Kundur Two-Area system, each synchronous generator is represented by 10 smaller units, each of 90 MVA with a $20-\mathrm{kV}$ rating. The inertia of the smaller units is the same as the aggregated system inertia, that is, $6.5 \mathrm{~s}$ for generating units 1 and 2 and $6.175 \mathrm{~s}$ for generating units 3 and 4 . This complies with the aggregated inertia concept represented by the following equation:

$$
H_{\text {agg }}=\frac{\sum_{i=1}^{n} H_{i} \cdot S_{i}}{S_{\text {tot }}}
$$

In this work, different penetration levels of DERs on the distribution feeder are created to properly identify the impact of DERs on the BES. As DERs replace synchronous machines, the reactive power reserve in a BES decreases, which might lead to a lower reactive power margin and a lower system voltage. There are two approaches currently used to consider the loss of the system inertia that results when DERs displace synchronous machines. One approach simply reduces the inertia of all of the machines proportionally; and the other approach disconnects certain generators from the system as the DER penetration reaches a certain threshold. Both approaches are able to emulate the decommissioning of conventional synchronous generators. The second approach is adopted in this work. 


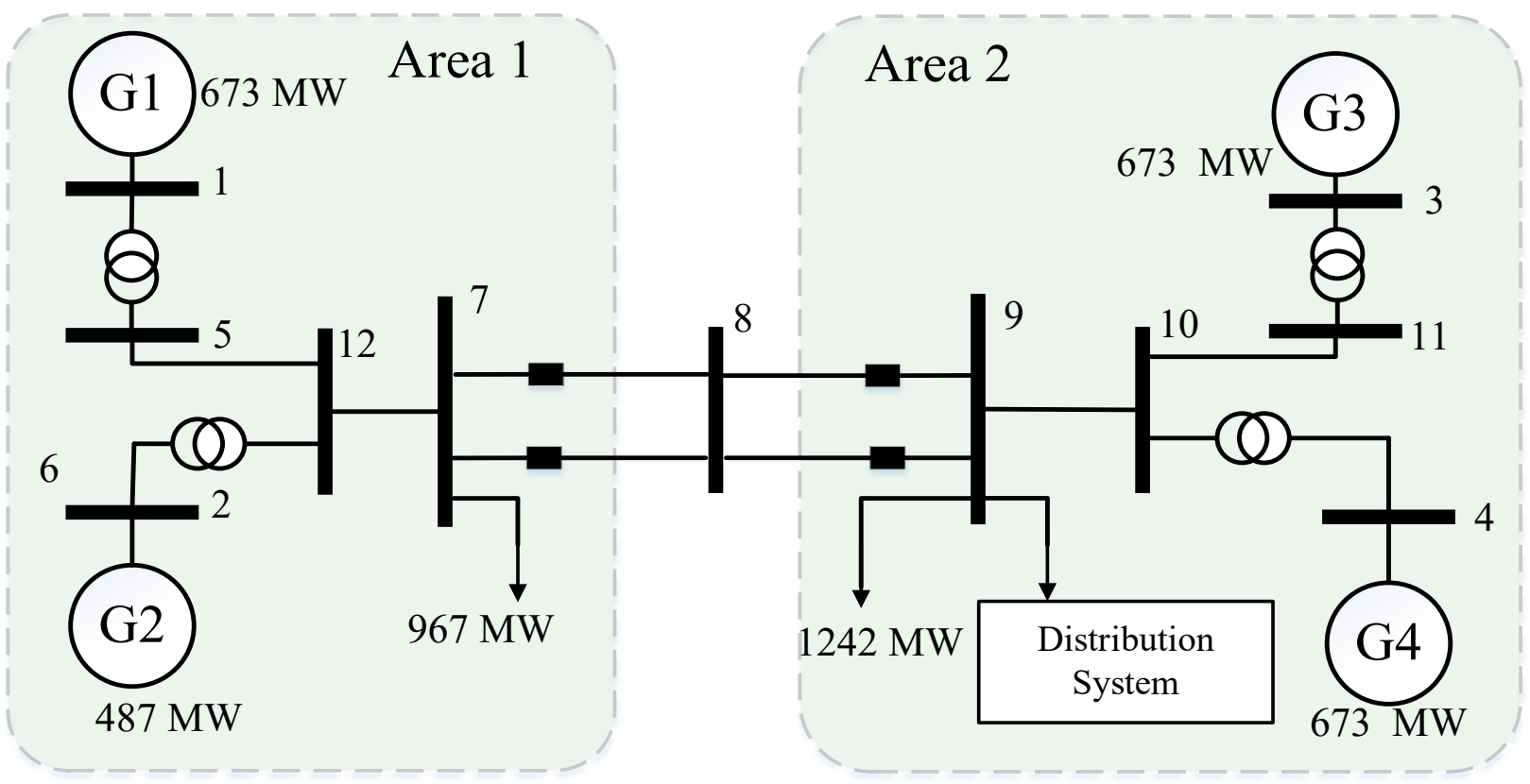

Figure 1 Schematic Diagram of the Modified Kundur Two-Area System.

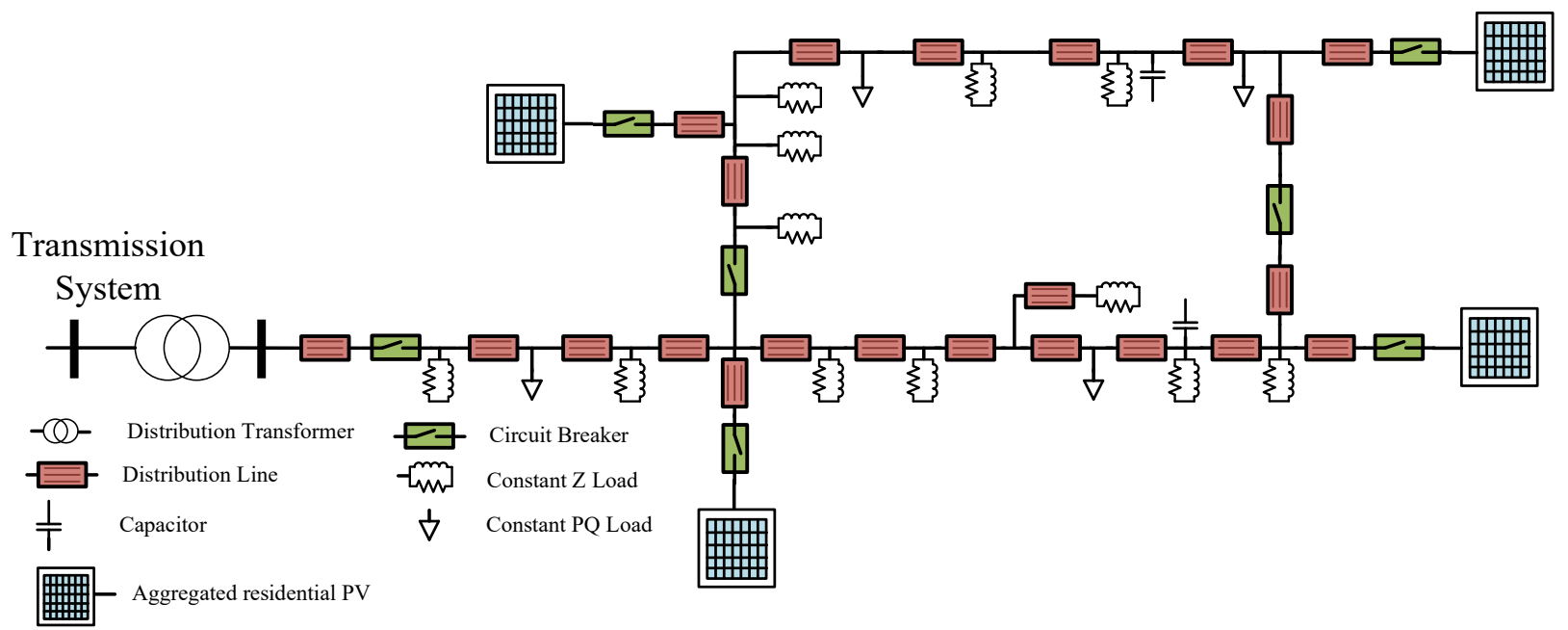

Figure 2 Schematic Diagram of the Test Distribution Feeder.

\subsubsection{Full Modeling of Distribution Systems}

In this approach, the non-aggregated distribution system and individual DERs are modeled to reflect their dynamic and steady-state behaviors for BES planning and operational studies. A representative distribution system, the modified IEEE 34-node test feeder shown in Figure 2, which hosts a few distributed PV systems, is developed in this work. The details of the distribution feeder can be obtained from [1]. Please note that the scale of PV systems used in this model is not at residential PV levels, which are usually connected at the low voltage side of the 
service transformers and range from a few kilowatts to $100 \mathrm{~kW}$. The distributed PV systems, in this work, represent an aggregation of multiple residential-scale PV systems connected at the primary distribution voltage level.

The full distribution system model is then connected to a transmission system model. The transmission system model and the full distribution system model as a whole, or the T\&D combined model, are shown in Figure 3. In the T\&D combined model, part of the load at bus \#9 in the BES is replaced with distribution feeders. To reduce the computational burden and simulation runtime while not affecting the impact study results, the following change is made: instead of using multiple different feeders, the representative distribution feeder connected to the distribution substation is scaled up multiple times using a current source and assuming that each feeder in the distribution system has the same response and the same load and DER penetration. Specifically, according to the total original transmission load and the capacity of the simulated distribution feeder, a distribution system with $n$ number of identical feeders, can be calculated to match the original load at the transmission bus. Then, a current source injection is used to scale up the modeled feeder $n-1$ times at the substation transformer (see Figure 3 ) to represent another $n-1$ feeders.

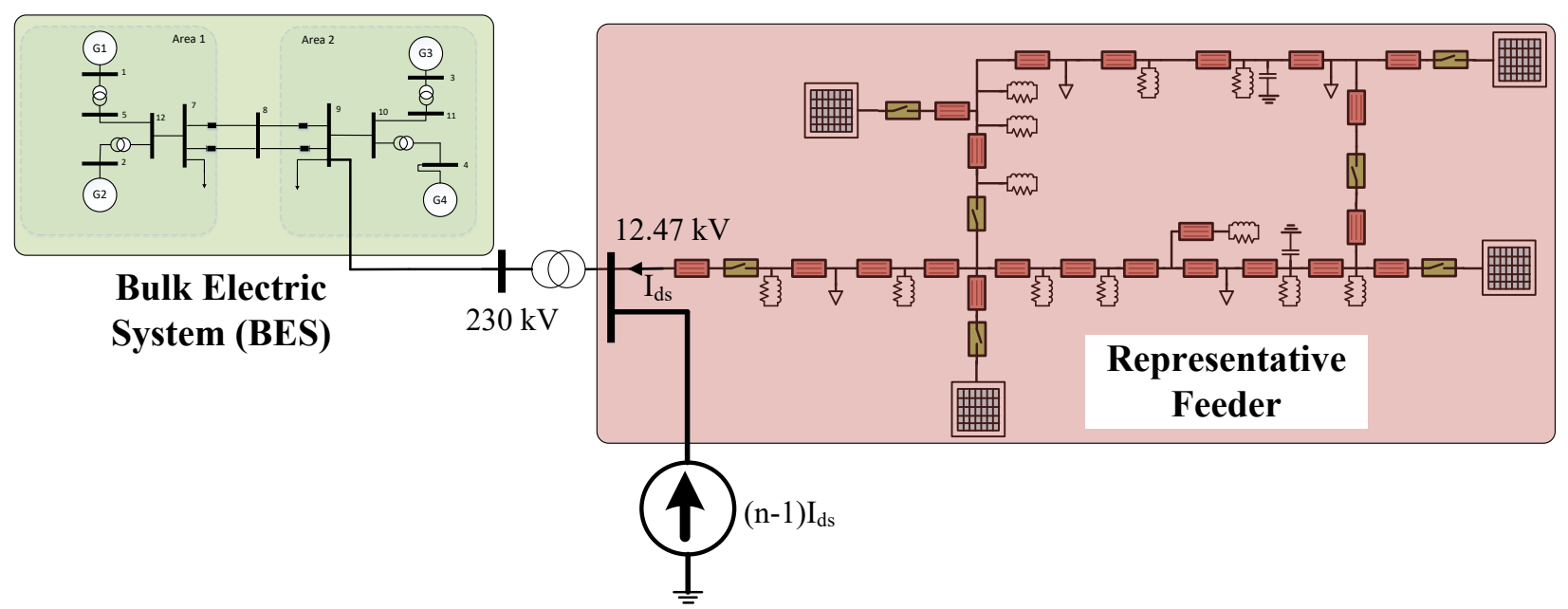

Figure 3 Schematic Diagram of a Transmission System with Partial Load Replaced by Distribution Feeders Integrated with DERs.

\subsubsection{PV System Modeling in Full Distribution System Models}

As shown in Figure 2 and Figure 3, four PV systems are integrated into the distribution feeder model at four different locations. In this study, the following characteristics for the PV systems are considered:

- $\quad$ PV systems are assumed to be operating at the maximum power point (MPP). The irradiance and the panel temperature are assumed to be constant; thus, the PV systems deliver constant real power to the alternating current (AC) side for the case studies. 
- PV systems are connected to a distribution node via a transformer that has a rating equal to the volt-ampere (VA) rating of the PV system.

- Each PV system has its own circuit breaker and protection logic scheme, which is implemented according to IEEE Standard 1547a-2014 [4] (see Table 2 and 3 ).

- An average dynamic model of the grid-connected inverter is used instead of one that is detailed ${ }^{1}$ given that the electromagnetic transients associated with the insulated-gate bipolar transistor (IGBT) switching are not of concern. The average model incorporates all of the control and inverter response dynamics but excludes the switching dynamics and associated harmonics.

- $\quad$ The time step used for the simulation is $50 \mu \mathrm{s}$.

- A standard vector control approach is used for the PV inverter control, which is in the PQ (i.e., active power and reactive power) control mode for the case studies below. The associated inverter control for the detailed mode of DER is shown in Figure 4.

Table 2 DER Interconnection System Response to Abnormal Voltages as per IEEE 1547a-2014.

\begin{tabular}{cc}
\hline $\begin{array}{c}\text { Voltage Range } \\
\text { \% of Base Voltage) }\end{array}$ & Clearing Time (seconds) \\
\hline$V<45$ & 0.16 \\
$45 \leq V<60$ & 1.00 \\
$60<V<88$ & 2.00 \\
$110 \leq V<120$ & 1.00 \\
$V \geq 120$ & 0.16 \\
\hline
\end{tabular}

${ }^{1}$ A detailed inverter model is used to model each of the switching components in the inverter to perform electromagnetic transient analysis and harmonic analysis. 
Table 3 DER Interconnection System Response to Abnormal Frequencies as per IEEE 1547a-2014.

\begin{tabular}{lcc}
\hline \multicolumn{1}{c}{ Function } & Frequency $(\mathrm{Hz})$ & $\begin{array}{c}\text { Clearing Time } \\
\text { (seconds) }\end{array}$ \\
\hline & & \\
Under Frequency (UF) 1 & $<57.0$ & 0.16 \\
UF 2 & $<59.5$ & 2.00 \\
Over Frequency (OF) 1 & $>60.5$ & 2.00 \\
OF 2 & $>62.0$ & 0.16 \\
\hline
\end{tabular}

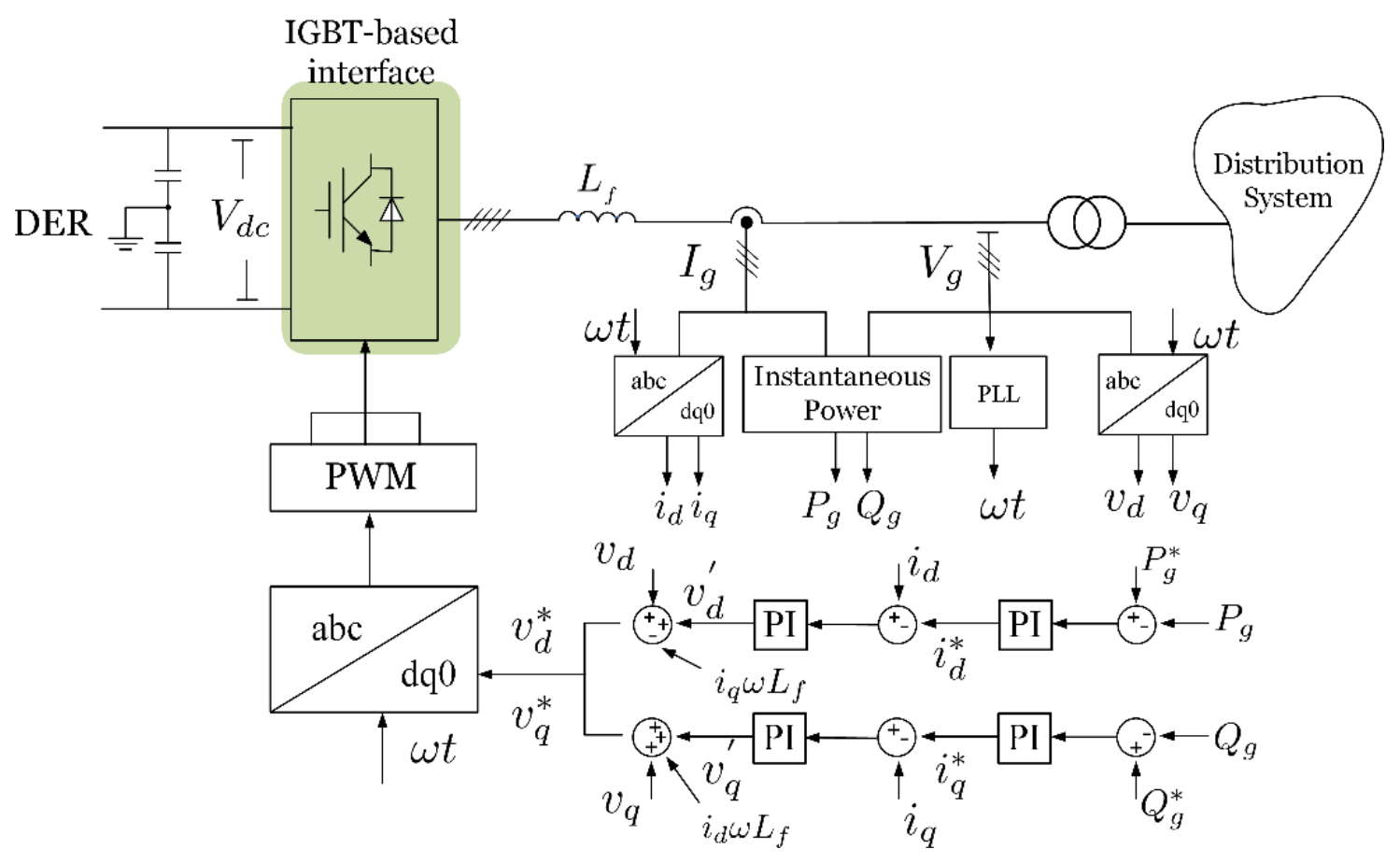

Figure 4 Schematic Diagram of the Inverter-Interfaced DER Model [5].

\subsubsection{Aggregated Modeling of Distribution Systems}

Conventionally, the DERs have been treated as the negative loads for steady-state analysis and used to be netted with load for BES planning and steady-state studies. There used to be no unique representation of DERs in both steady-state and dynamic studies. More recently, with the increasing penetration of DERs on the distribution system, for steady-state analysis purposes, the recommended model consists of a substation transformer connected to an equivalent load, and an equivalent PQ generator through an equivalent feeder impedance. This representation of distribution system with DERs works well for steady-state studies like load flow analyses but may not be enough for dynamic studies. Western Electricity Coordinating Council (WECC) has developed an equivalent model that represents the distribution system load and distributed PV system for dynamic studies, namely, the CMPLDW [6] and PVD1 models [7], respectively. 
In this report, following the WECC recommendation for the aggregated representation of a distribution system, a static load equal to the power drawn by a representative distribution system is used. The dynamic motor loads in the CMPLDW model are not considered. The losses in the system are modeled using an equivalent feeder impedance. The aggregate load model and the equivalent feeder segment are the same for both steady-state and dynamic aggregated distribution models. In addition, for the distributed PV system, a static aggregate DER model (PQ generator) is used in the steady-state aggregated distribution system model. The PVD1 model developed by WECC is utilized for the dynamic aggregate DER model in the dynamic aggregated distribution system model. The aggregate static/dynamic DER model, in conjunction with a substation transformer, an aggregate load model, and an equivalent feeder segment is collectively referred to as the aggregated distribution system model.

The aggregated distribution system model for the IEEE 34-node test feeder shown in Figure 2 is developed in this work. The schematic diagram of both steady-state and dynamic aggregated distribution system models is shown in Figure 5. The procedure to obtain the parameters for the equivalent load and equivalent feeder segment is referenced in Appendix 0. The aggregated distribution system model is connected to a transmission system model to enable studying the impact of DERs on the BES.

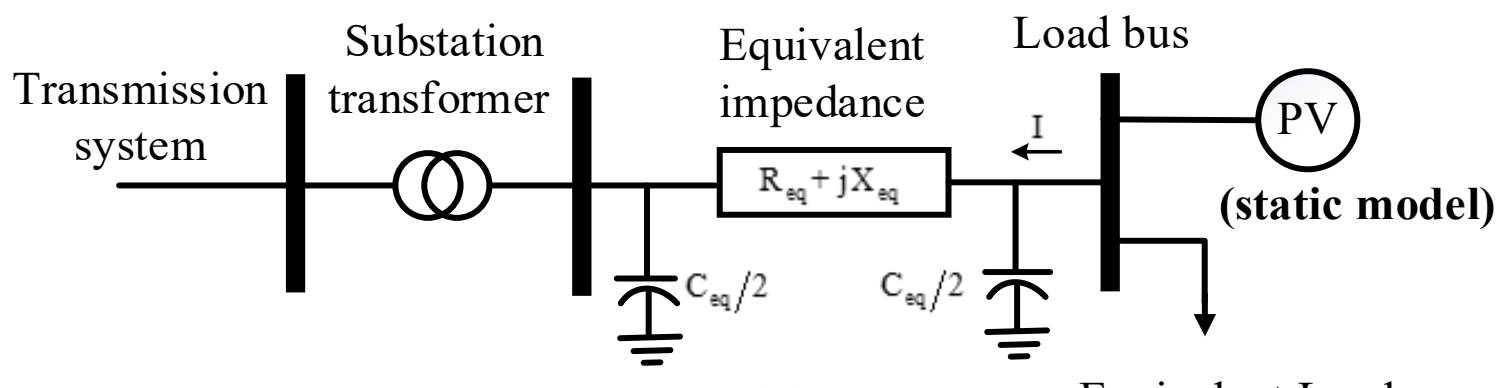

(a) Equivalent Load

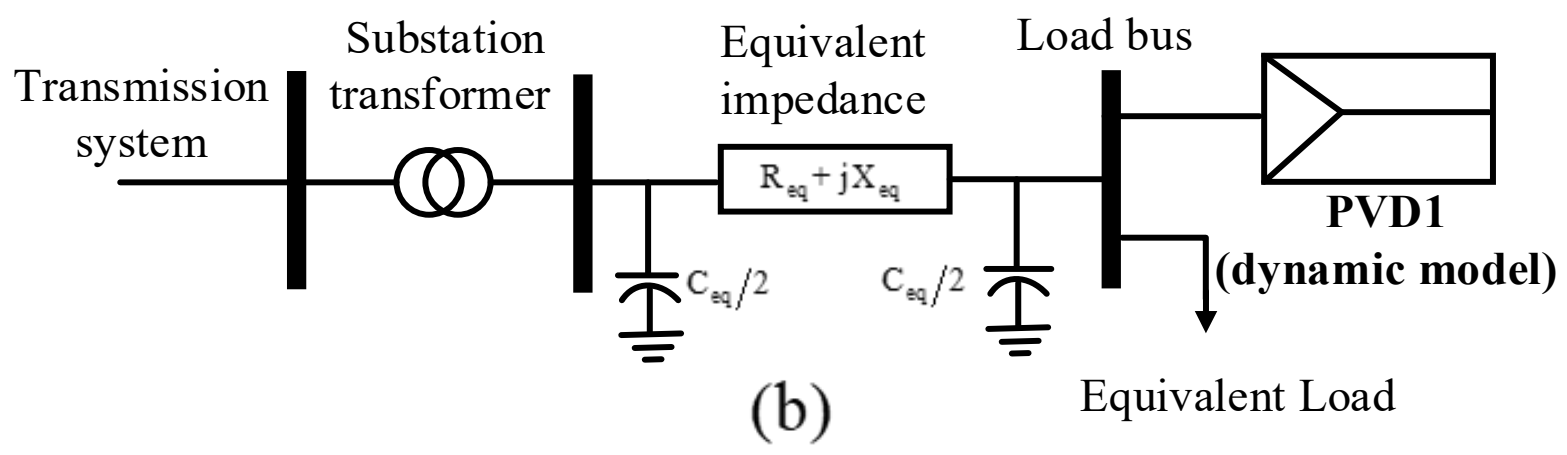

Figure 5 Aggregated Steady-State (a) and Dynamic (b) Model of the Distribution System Integrated with Distributed PV Systems. 


\subsubsection{Aggregated Distributed PV Model (PVD1 Model)}

In this work, the PVD1 model is adopted to aggregate the distributed PV generation in the distribution system. PVD1 [7] is a dynamic equivalent model representing an average response of all of the distributed PVs in the distribution system. The block diagram representation of the PVD1 generator model is shown in Figure 6. The following points are worth noting about the PVD1 model used in this work:

- The active power control loop and reactive power control loop provide the active and reactive current injection to the network, respectively.

- The active and reactive current commands are subject to a dynamic current limiting, with user-selectable priority between active and reactive current. In the cases studied, the active power output from the PV inverter is prioritized over reactive power output. This treatment means that the maximum amount of reactive power support that can be obtained is dependent on the active power output from the PV inverter.

- The active current command is derived from a reference active power, and the reactive current command is obtained from the sum of the reactive power output from the Volt-VAR curve for terminal voltage regulation and reference reactive power. This setup is illustrated in Figure 6.

- In the case studies, the reactive power output from the PV is assumed to be zero. Therefore, the reactive power reference is zero, and the Volt-VAR droop control is not in effect.

- The total PV power output at different locations of the distribution system are added up to obtain the equivalent power output of the PVD1 model.

- The model consists of protective functions, which reduce the output of the PVD1 when the system operates outside of the user-specified dead band on voltage and frequency. If the performance of legacy DERs (tripping) and modern DERs (ride-through) is modeled by the use of a single instance of the PVD1 model, values for the "Vrflag" and "Frflag," equal either 0 or 1. Fraction values (i.e., between 0 and 1) of "Vrflag" and "Frflag" may be used to represent partial tripping from a mixture of legacy and modern DERs with different interconnection standards.

- The recovery of generation is dependent on whether the occurrence of a voltage or frequency excursions reverse and return toward the dead band, and the proportion is determined by the user-defined flags (i.e., "Vrflag" and "Frflag"). For example, a "Vrflag" of 0.5 means that $50 \%$ of the PV systems reconnect to the grid when the voltage recovers to nominal value following a voltage recursion. The effects of variation of values of "Vrflag" and "Frflag" are also explained in the case studies. 


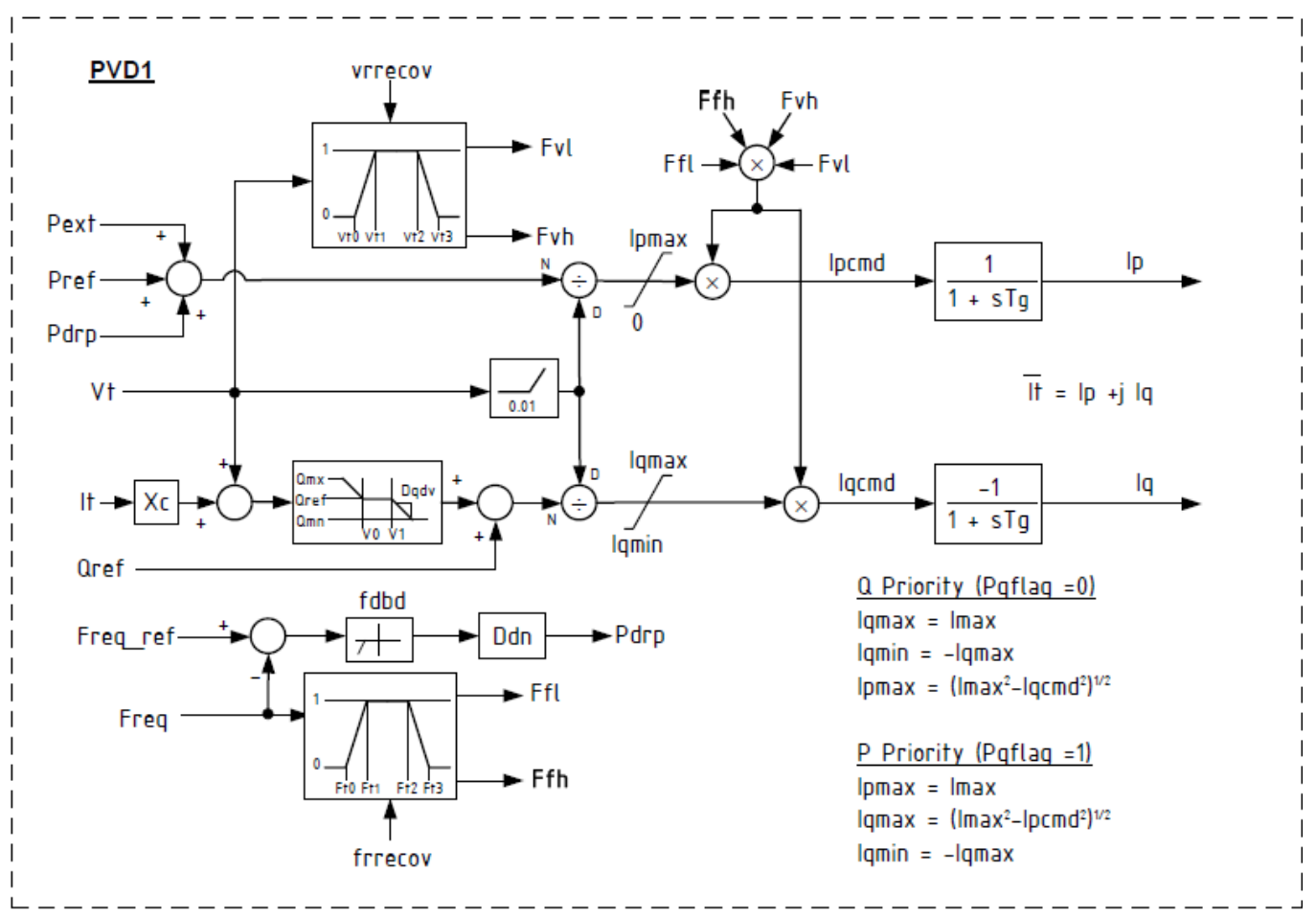

Figure 6 Schematic Diagram of PVD1 [7].

The parameters for the PVD1 model used in the case studies are shown in Table 4.

Table 4 PVD1 Parameters Used in the Case Studies.

\begin{tabular}{lll}
\hline & \multicolumn{1}{c}{ Description } & Values \\
\hline Name & & \\
Xc & Line drop compensation reactance (pu on MVAbase) & 0.0 \\
Imax & Apparent current limit (pu on MVAbase) & 1.3 \\
Vt0 & Voltage tripping response curve point 0 (pu) & 0.88 \\
Vt1 & Voltage tripping response curve point 1 (pu) & 0.90 \\
Vt2 & Voltage tripping response curve point 2 (pu) & 1.10 \\
Vt3 & Voltage tripping response curve point 3 (pu) & 1.20 \\
Vrflag & Voltage tripping is latching (0) or partially self-resetting $(>0$ and $\leq 1)$ & $0.0 / 1.0$ \\
Ft0 & Frequency tripping response curve point 0 (Hz) & 59.5 \\
Ft1 & Frequency tripping response curve point 1 (Hz) & 59.7 \\
Ft2 & Frequency tripping response curve point 2 (Hz) & 60.3 \\
Ft3 & Frequency tripping response curve point 3 (Hz) & 60.5 \\
Frflag & Frequency tripping is latching (0) or partially self-resetting $(>0$ and $\leq 1)$ & $0.0 / 1.0$ \\
Qref & Reactive power reference (pu) & 0.0 \\
Tg & Inverter current lag time constant (seconds) & 0.02 \\
\hline
\end{tabular}




\subsection{PERFORMANCE COMPARISON OF TWO DISTRIBUTION SYSTEM MODELING APPROACHES IN BES IMPACT STUDIES}

In this subsection, case studies are carried out to investigate the performance of aggregated distribution system models against full distribution system models. In the case studies, the PV penetration level for the representative distribution system is close to $75 \%$ of the distribution system load; for the overall BES load, it is close to $15 \%$. In the case studies, when DER penetration increases, generation capacity and overall inertia in the transmission system are scaled down proportionally to reflect the effect of DERs displacing conventional generators.

The operating scenarios considered in the case studies are summarized in Table 5.

Table 5 Operating Conditions for the T\&D Networks for the Case Studies.

\begin{tabular}{|c|c|c|c|c|}
\hline \multicolumn{5}{|l|}{ Generator Data } \\
\hline & G1 & $\mathrm{G} 2$ & G3 & G4 \\
\hline Power Output (MW) & 673.2 & 486.85 & 673.2 & 673.2 \\
\hline Inertia $(\mathrm{s})$ & 6.25 & 6.25 & 5.95 & 5.95 \\
\hline $\mathrm{AGC}^{\mathrm{a}}$ & Disabled & Disabled & Disabled & Disabled \\
\hline PSS & Enabled & Enabled & Enabled & Enabled \\
\hline \multicolumn{5}{|l|}{ Load Data } \\
\hline Load in Area 1 & \multicolumn{4}{|c|}{967 MW, 100 MVAR (inductive), and 387 MVAR (capacitive) } \\
\hline Load in Area 2 & \multicolumn{4}{|c|}{$1242 \mathrm{MW}, 100 \mathrm{MVAR}$ (inductive), and 537 MVAR (capacitive) } \\
\hline $\begin{array}{l}\text { Total Load in } \\
\text { Distribution System } \\
\text { Connected in bus } 9\end{array}$ & \multicolumn{4}{|c|}{ 505.2 MW, 80.4 MVAR (inductive) } \\
\hline \multicolumn{5}{|l|}{ PVD1 Parameters } \\
\hline Generation & \multicolumn{4}{|c|}{405 MW, 0 MVAR (reactive power output disabled) } \\
\hline Protection Settings & \multicolumn{4}{|c|}{ Refer to Table 4} \\
\hline \multicolumn{5}{|l|}{ Detailed PV Model } \\
\hline Generation & \multicolumn{4}{|c|}{ 4*3.375 MW, 0 MVAR (reactive power output disabled) } \\
\hline Protection Settings & \multicolumn{4}{|c|}{ Refer to Appendix A } \\
\hline No. of Feeders $(n)$ & \multicolumn{4}{|c|}{30} \\
\hline
\end{tabular}

${ }^{\text {a }} \mathrm{AGC}=$ Automatic Generation Control, PSS $=$ Power System Stabilizers. 


\subsubsection{Performance Comparison of Two Modeling Approaches though a Load Step Change Case Study}

In this case study, a step increase of $100 \mathrm{MW}$ in load is introduced to the overall load in Area 2. This event causes the system frequency to deviate from its nominal value, and the initial response depends on the inertia of the synchronous machines connected to the BES.

For this case study, both "Vrflag" and "Frflag" for the PVD1 model are set to 0 . This choice indicates that, once part or all of the PV system trips either due to voltage or frequency violation, they do not connect back automatically - even after the fault is cleared and the system voltage and frequency recover. In this study, the T\&D combined model is also referred to as the "non-aggregated model" to contrast with the aggregated modeling approach.

Figure 7 delineates the system frequency deviation from $60 \mathrm{~Hz}$ following a 100-MW step change in load in the BES. The total simulation time is 80 seconds; and the load change is introduced at $20 \mathrm{~s}$. The results compare the system frequency deviation from $60 \mathrm{~Hz}$ considering both the full distribution system model and the aggregated distribution system model. As the model shows, the responses of both models are comparable to one another.

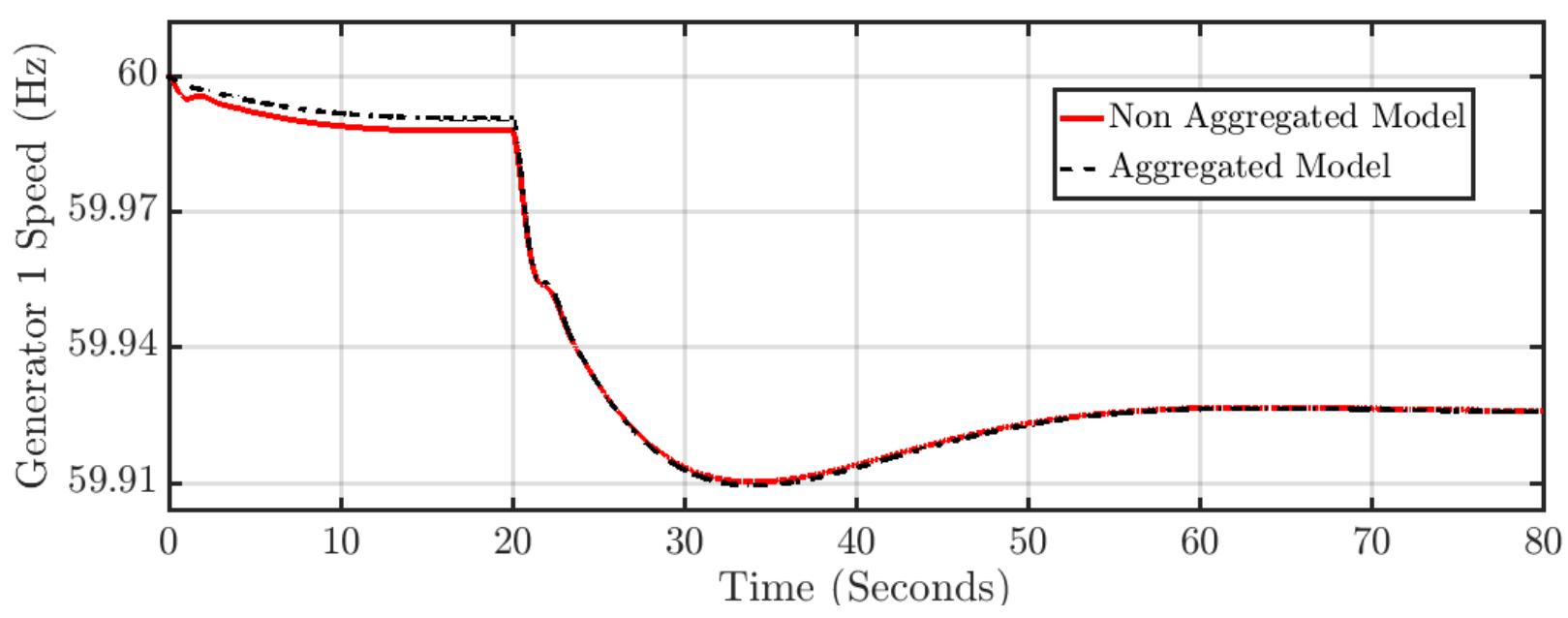

Figure 7 System Frequency Deviation from a Load Increase of 100 MW for Aggregated and Full Distribution System Models.

Figure 8 shows the substation power flow comparison for the aggregated distribution system model and the full distribution system model. As the figure shows, both models settle down to the same power level following the disturbance. The results in Figure 7 and Figure 8 validate the aggregation approach utilized to arrive at the parameters of the aggregated models. 


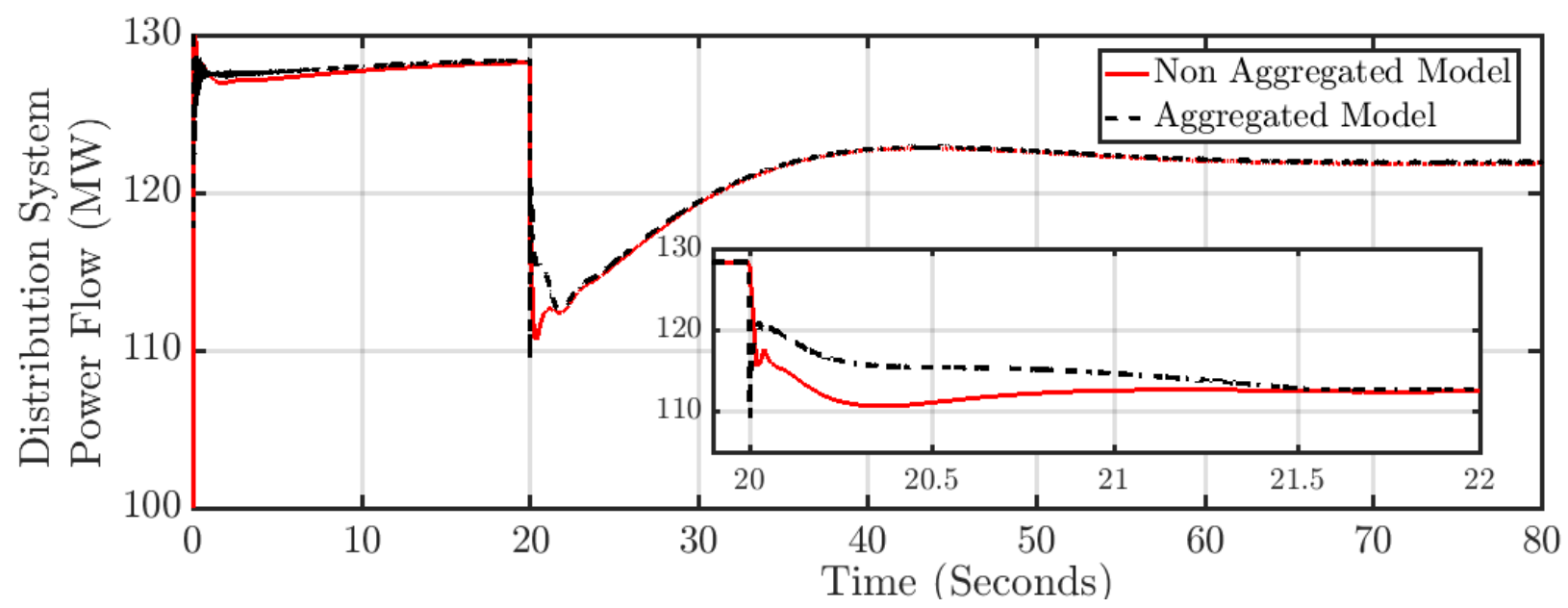

Figure 8 Substation Power Flow Composition for Aggregated and Full Distribution System Models.

Figure 9 shows the comparison of the change in the PV system voltage at the point of common coupling (PCC) following the load change. For the full model, all four distributed PV systems are considered. The responses using both models are similar, as Figure 9 shows.

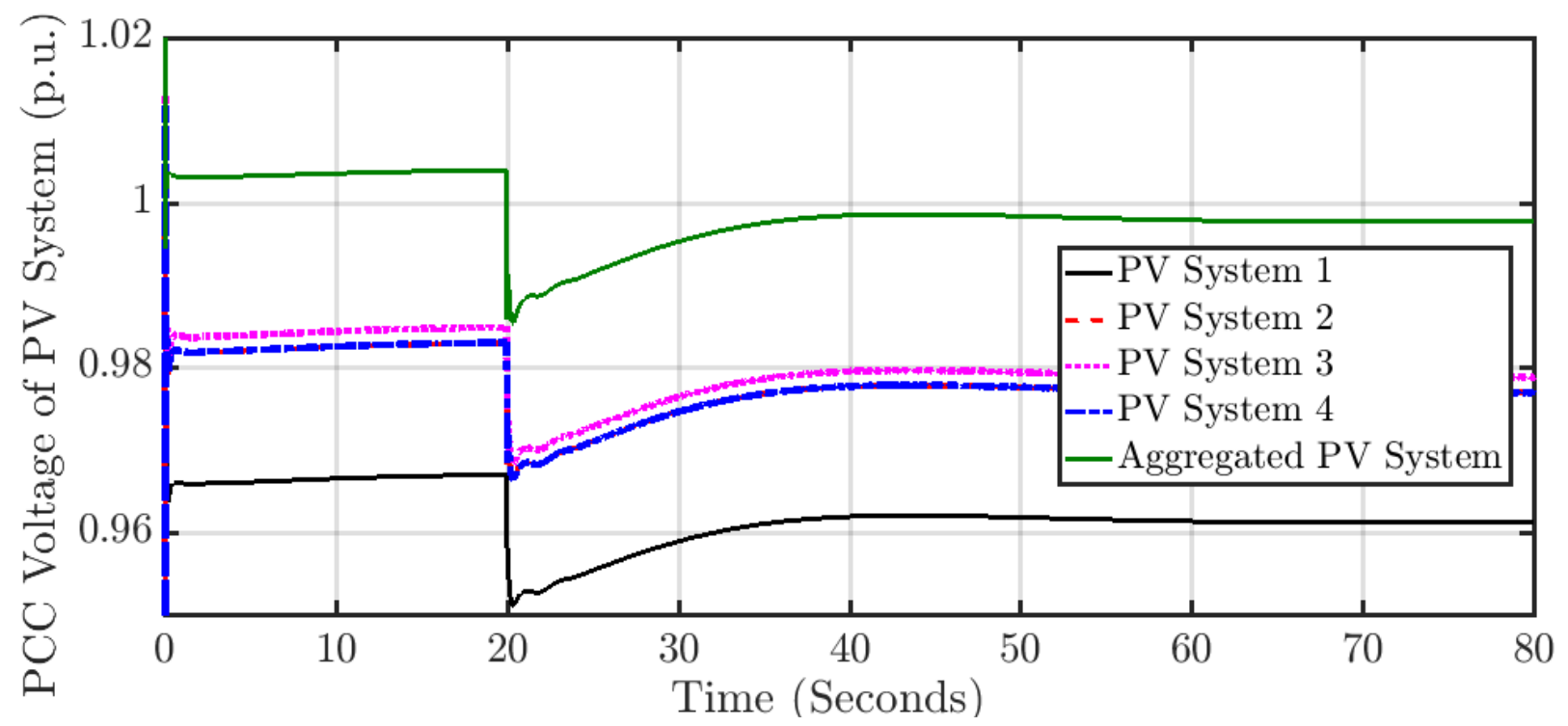

Figure 9 PV System PCC Voltage Deviation Comparison with Aggregated and Full Distribution System Models. 
Figure 10 compares the frequency at the PCC of the distributed PV systems with that of the PVD1 model. The responses of both types of models are comparable to one another. It should also be noted that for this test case, the protection system that is implemented (both frequency and voltage; see Table 4 for PVD1 and Table 2 and Table 3 for individual PV systems) in the DER model for both the detailed PV system and the PVD1 is not triggered, as both the system voltage and frequency stay within the allowed ranges.

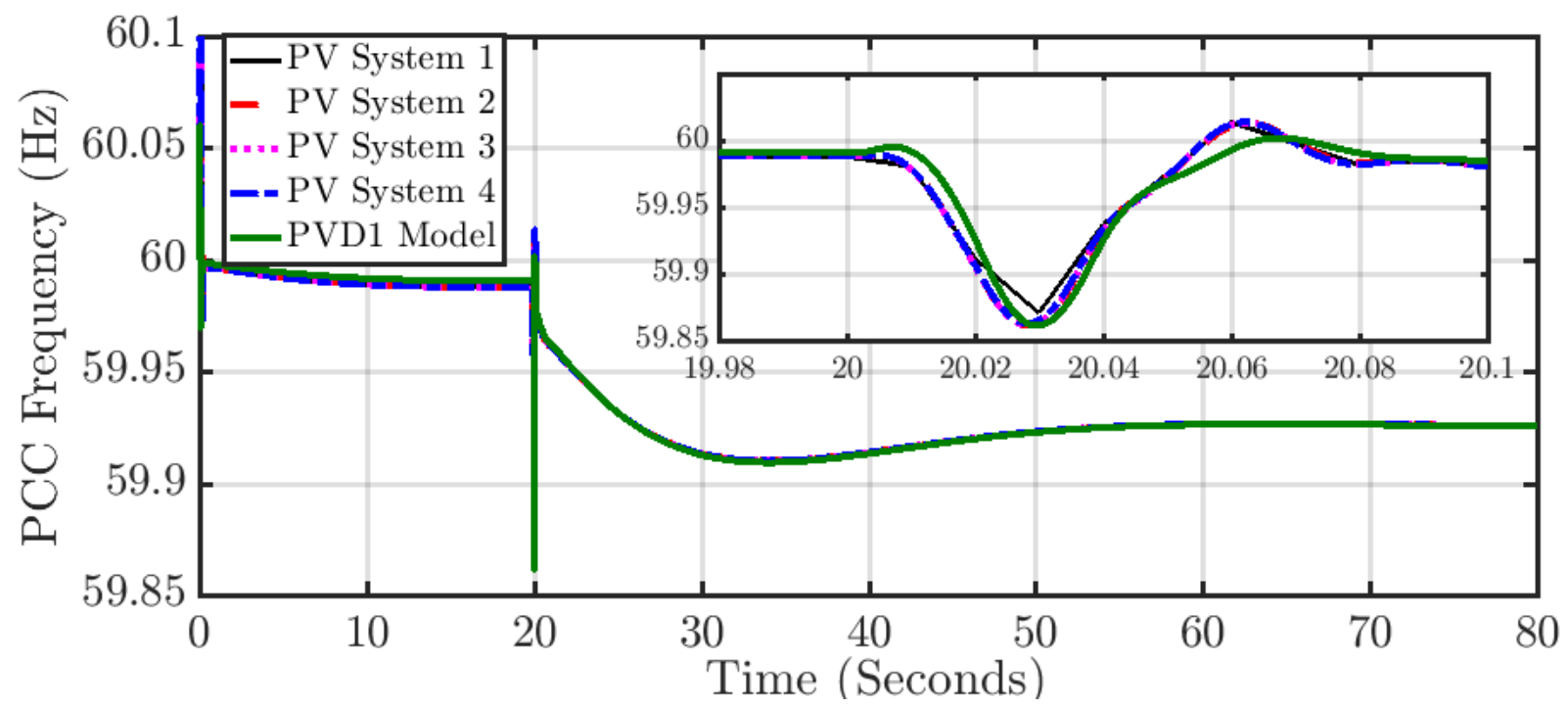

Figure 10 PCC Frequency Comparison for Distributed PV Systems in the Full Model and for the PVD1 in the Aggregated Model.

\subsubsection{Performance Comparison of Two Modeling Approaches through a Transmission System Fault Case Study}

In this case study, a self-clearing, three-phase-to-ground fault lasting for 12 cycles is applied at the middle of the tie-line connecting Areas 1 and 2 at bus 8 (see Figure 1). This event causes the system frequency and voltage to deviate from its nominal value and activates the protection system implemented in DERs. In this case, the "Vrflag" and "Frflag" for the PVD1 model are set to " $0 . "$

Figure 11 shows the frequency response for generator 1 (G1 in Figure 1) when an aggregated distribution system model is connected to the BES and when the full distribution system model is connected to the BES. It is of note that the nature of the response with both models is identical. However, the frequency nadir for the full model is slightly higher than for the aggregated model. 


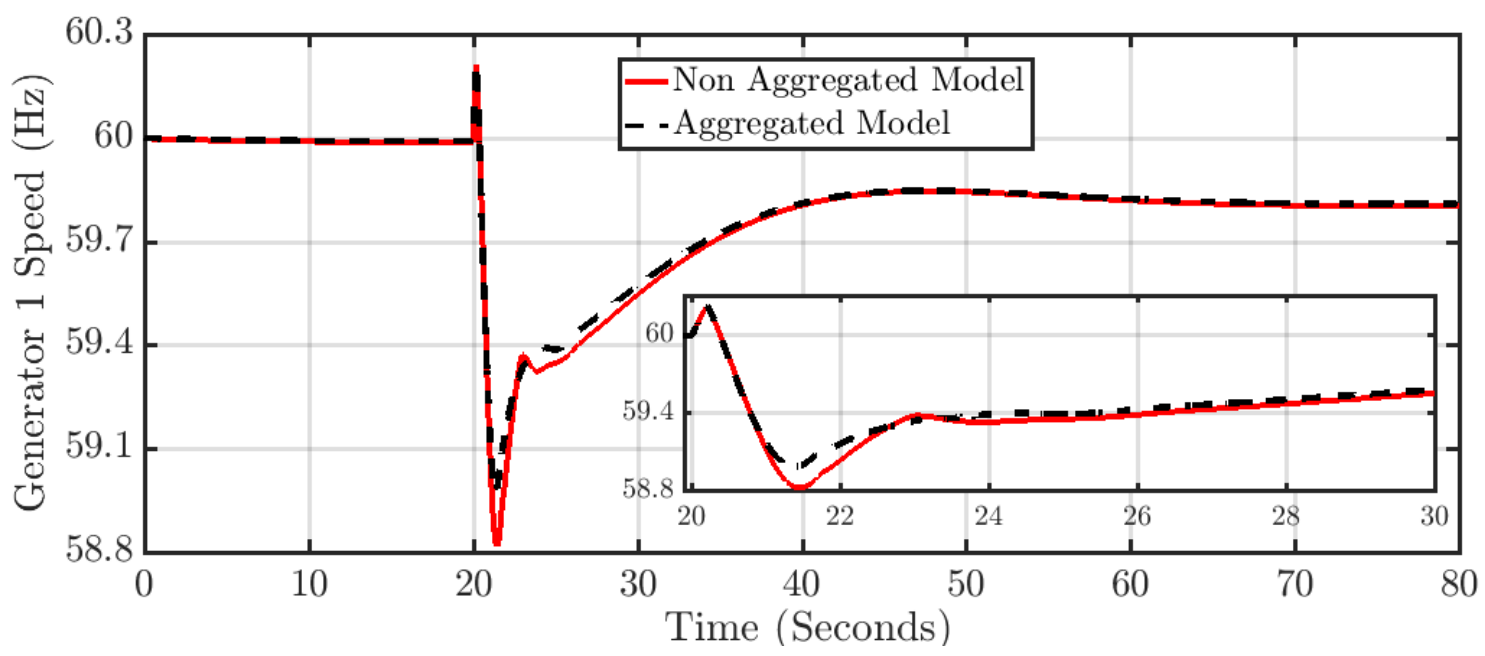

Figure 11 System Frequency Deviation Following a Self-Clearing, Three-Phase Bolted Fault at the Middle of the Tie-Line for Aggregated and Full Distribution System Models.

Figure 12 compares the substation power flow for the two models considered. With the full model, note that following the fault, the distribution system first draws in a higher amount of power from the BES; and as the fault clears, it tends to revert to the pre-fault condition.

However, because of the low frequency detected at the PCC terminals, at around $22.6 \mathrm{~s}$ all of the distributed PV systems disconnect. As the PV systems disconnect, Figure 12 shows that the full system then draws in around $467 \mathrm{MW}$ of power as the aggregated model.

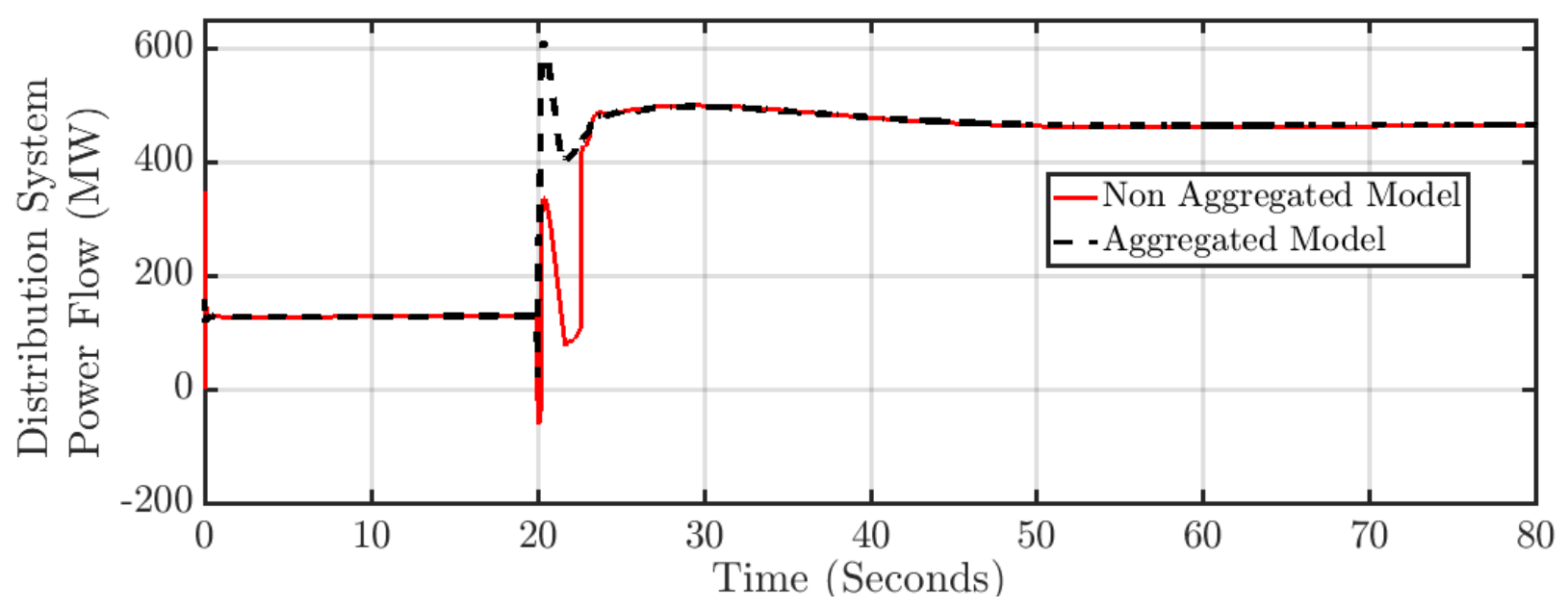

Figure 12 Substation Power Flow Composition for Aggregated and Full Distribution System Models.

Figure 13 compares the PCC frequency of the distributed PVs with the PCC frequency of the PVD1 model. The responses are comparable to one another; however, for the distributed $\mathrm{PVs}$, a second drop in frequency is observed around $22.6 \mathrm{~s}$, which is associated with the disconnection of PV systems from the distribution system resulting from a low-frequency protection trigger. 


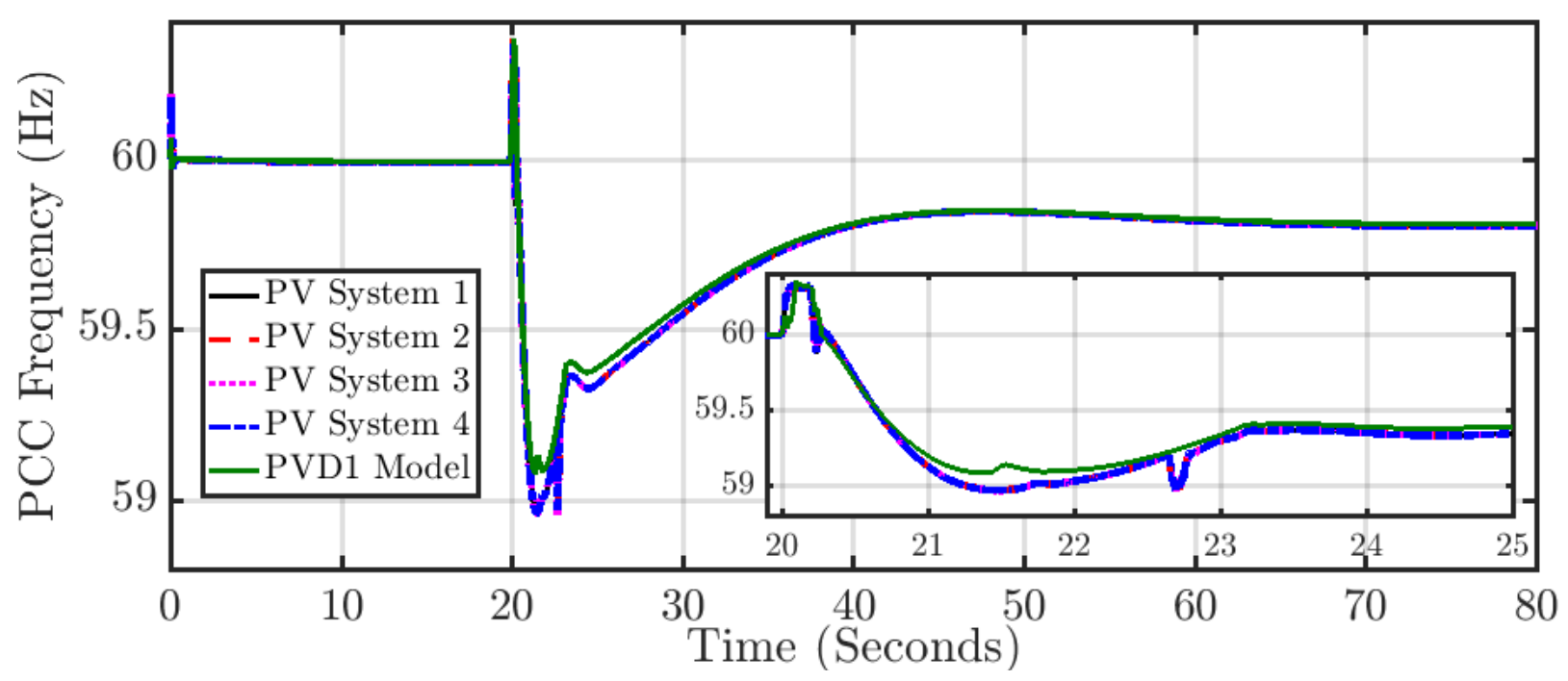

Figure 13 PCC Frequency Comparison for Distributed PV Systems in Full Model and PVD1 in Aggregated Model.

Figure 14 compares the power output of the distributed PVs with the power output of the PVD1 model. The power output is shown in p.u. at their respective base values. The PVD1 model's power output almost immediately drops down to zero following the fault. This result is expected as the PVD1 model does not have any time consideration for tripping offline from the voltage sag caused by the fault. However, for the distributed PV system, the PV system rides through the fault but trips at a later time because of the slower frequency recovery of the BES.

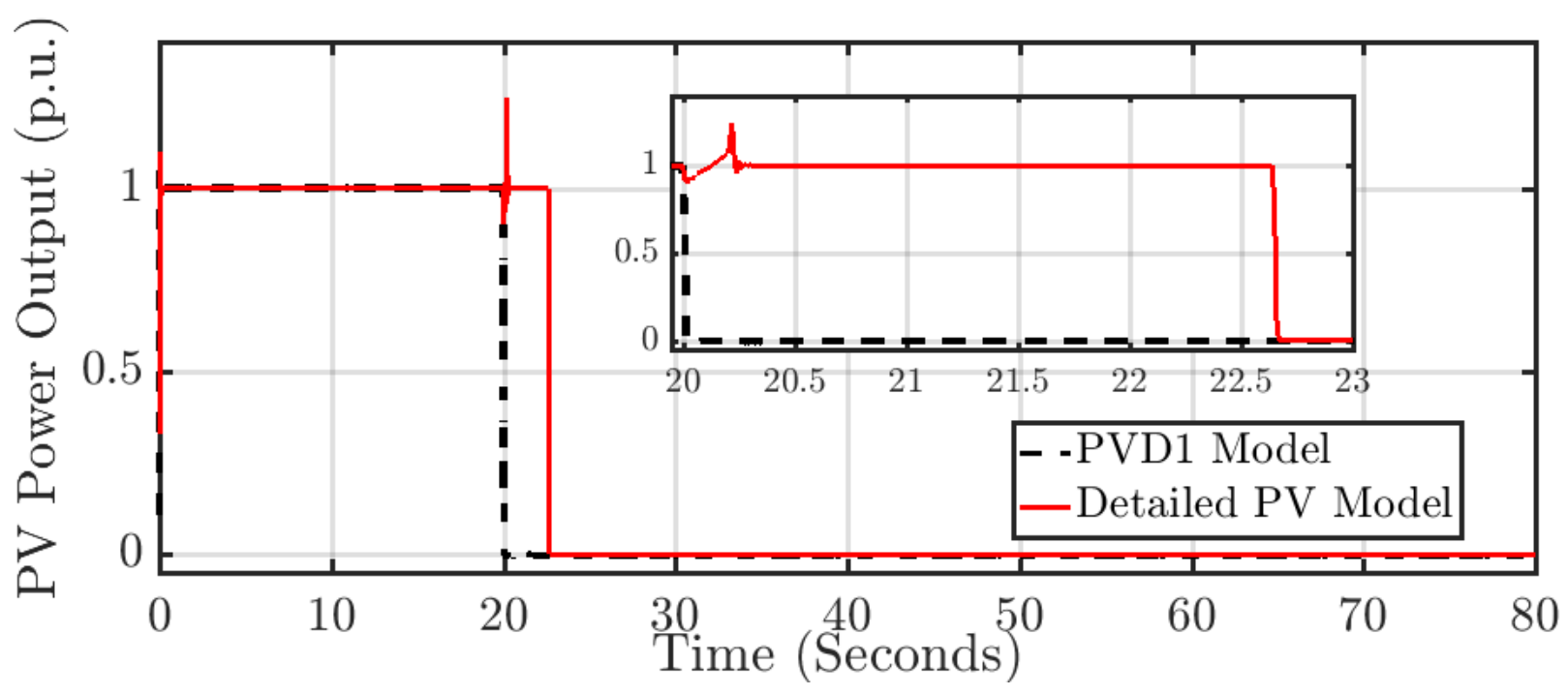

Figure 14 PV Output Comparison for Aggregated and Full Distribution System Models.

Figure 15 compares the PCC voltage for the PVD1 model with one of the distributed PV systems. It can be observed that the PCC voltage stays in the range between 0.60 p.u. to 0.88 p.u. 
for less than $2.0 \mathrm{~s}$ for both models. However, as mentioned concerning Figure 14, this lowvoltage event causes the PVD1 model to trip immediately, whereas the distributed PV system (with the IEEE 1547-a 2014 [4] incorporated) does not trip because of voltage deviation; rather, it trips later (after 22.5 seconds) because of frequency deviation. This result causes the PCC voltage response to be different for both models.

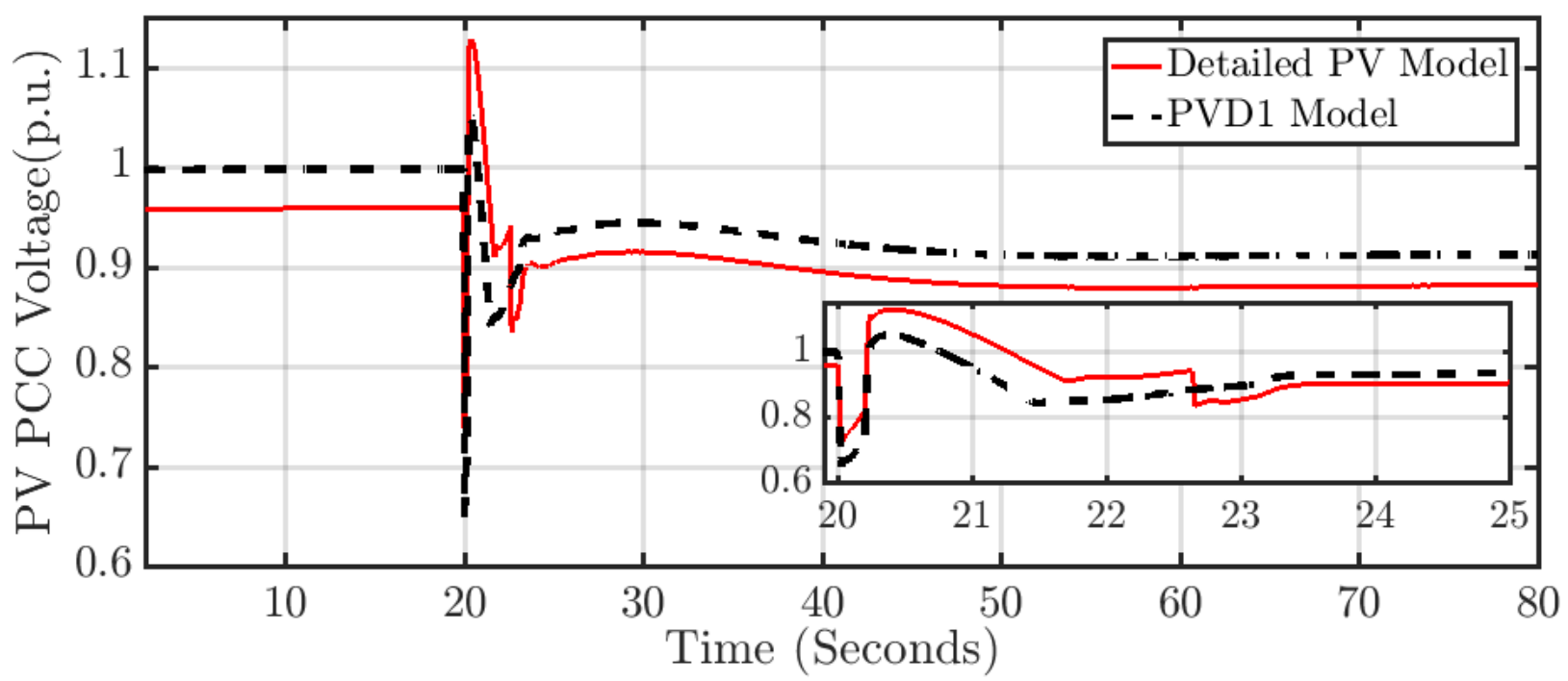

Figure 15 PV PCC Voltage for the Distributed PV Model and PVD1 Model.

Even though both the distributed PV systems and the PVD1 model disconnect from the grid, each model disconnects for different reasons.

Given the differences in the results observed in Figure 14 and Figure 15, our recommendation is to use the full modeling approach for impact studies to reach more accurate results. In the next chapters, the T\&D combined model of the full distribution system is utilized to study the impact of DERs on the BES. 


\section{DERS IMPACT ON AND CONTROL STRATEGIES TO IMPROVE FREQUENCY CONTROL OF THE BES}

In this chapter, a virtual-synchronous-generator-based control scheme is proposed for DERs, and the scheme's contribution to the BES inertial response is demonstrated in the first section. Then DERs' impacts on BES net load variability and ramping are studied; and mitigation schemes are discussed, implemented, and validated in the second section.

\subsection{CONTROL STRATEGIES FOR ENHANCING BES FREQUENCY RESPONSE}

\subsubsection{Fast Frequency Regulation for BES Contingencies}

In recent years, significant inverter-based, inertia-less renewable generation (e.g., photovoltaics and wind turbines) has been integrated into both bulk power transmission and distribution systems to improve the sustainability of electric power systems. The increasing penetration of these DERs displacing conventional synchronous generators (SGs) is rapidly changing the dynamics of large-scale power systems. The electric grid is subject to loss of inertia, voltage support, and oscillation damping [8]-[11]. These conditions occur because inverterinterfaced distributed generators (DGs) with conventional control methods, such as P-V droop control, still cannot provide sufficient inertia to support the frequency of the grid. Therefore, it will be harder for the system to recover from a frequency excursion event.

To solve this problem the idea of virtual synchronous generator (VSG) has been presented in [12], [13] in which the power electronics converter is mimicking the behavior of the SG. However, the implementation of virtual inertia in the literature varies based on the desired level of model complexity and application while the underlying concept is similar among various topologies. A detailed mathematical model which represents the dynamics of SG or simplifying the model by using only the swing equation are the two main solutions used for implementing virtual inertia [12], [14]. For example, Synchroverters are introduced in [15], [16] for inverter based DGs. Similar to Synchroverter approach, another topology is presented in the literature that uses the control loop to solve the swing equation in each cycle to emulate inertia, instead of using the full detailed model of the SG [17]. Other than these paper presenting the different topologies, VSG application is illustrated extensively in the literature. In [18] an enhanced VSG control is proposed in which the virtual stator reactance is adjusted to improve the active power oscillations during transient states. Furthermore, using inverse voltage droop control and ac bus voltage estimation, an accurate reactive power sharing is achieved. In [19], the dynamic characteristics of simple droop control and VSG are studied through deriving small signal equations in both islanded and grid connected mode and an inertial droop control is then proposed that inherits the advantages of droop control but also provides inertial support for the system. Voltage angle deviations (VADs) of generators with respect to the angle of the center of inertia are defined in [20] as a tool for transient stability assessment of the multi-VSG microgrid. To have a smooth transition during disturbances and keeping VADs within a specific range, VSG parameters are tuned using particle swarm optimization. In [21] the detailed parameters design of VSG is proposed and also the conditions to decouple active and reactive power loops are given. For avoiding VSG output voltage 
distortions, the author indicates that the bandwidth of the power loop should be smaller than twice the line frequency. In [22], the author proposed a virtual inertia strategy for DC micro grids through bidirectional grid connected converters to enhance the inertial response of DC micro grids and stabilize the DC bus voltage fluctuations. A fuzzy secondary controller-based VSG control scheme is proposed in [23] for voltage and frequency regulation in micro grids. [24] proposed a low voltage ride through control strategy for VSG control scheme that provides reactive power support under grid faults. The solution strategy for VSG working under unbalanced voltage conditions is discussed in [25]. A new VSG control is presented in [26] with capability to avoid harmonic interference and accurate control vector orientation process.

In this work, the VSG concept will be adopted, where the DG will provide virtual inertia by emulating the swing equation of a synchronous generator in the inverter-interfaced DGs control to improve the dynamic performance, especially the transient response of the system frequency.

Since the power electronic interfaces used in DGs have no rotating mass and damping, the inertial constant in the microgrid is reduced which results in increasing the rate of change of frequency (RoCoF) of the grid and may lead to the load shedding even under small disturbances in the system. Figure 16 shows the frequency curve of system with different amount of inertia in presence of a contingency. For the control and stability of these small scale power grids, a hierarchical algorithm including primary, secondary and tertiary control is introduced, similar to conventional grids. Droop control for voltage source inverters as an example for primary frequency control is discussed in [27], provide barely any inertia/damping support for the grid.

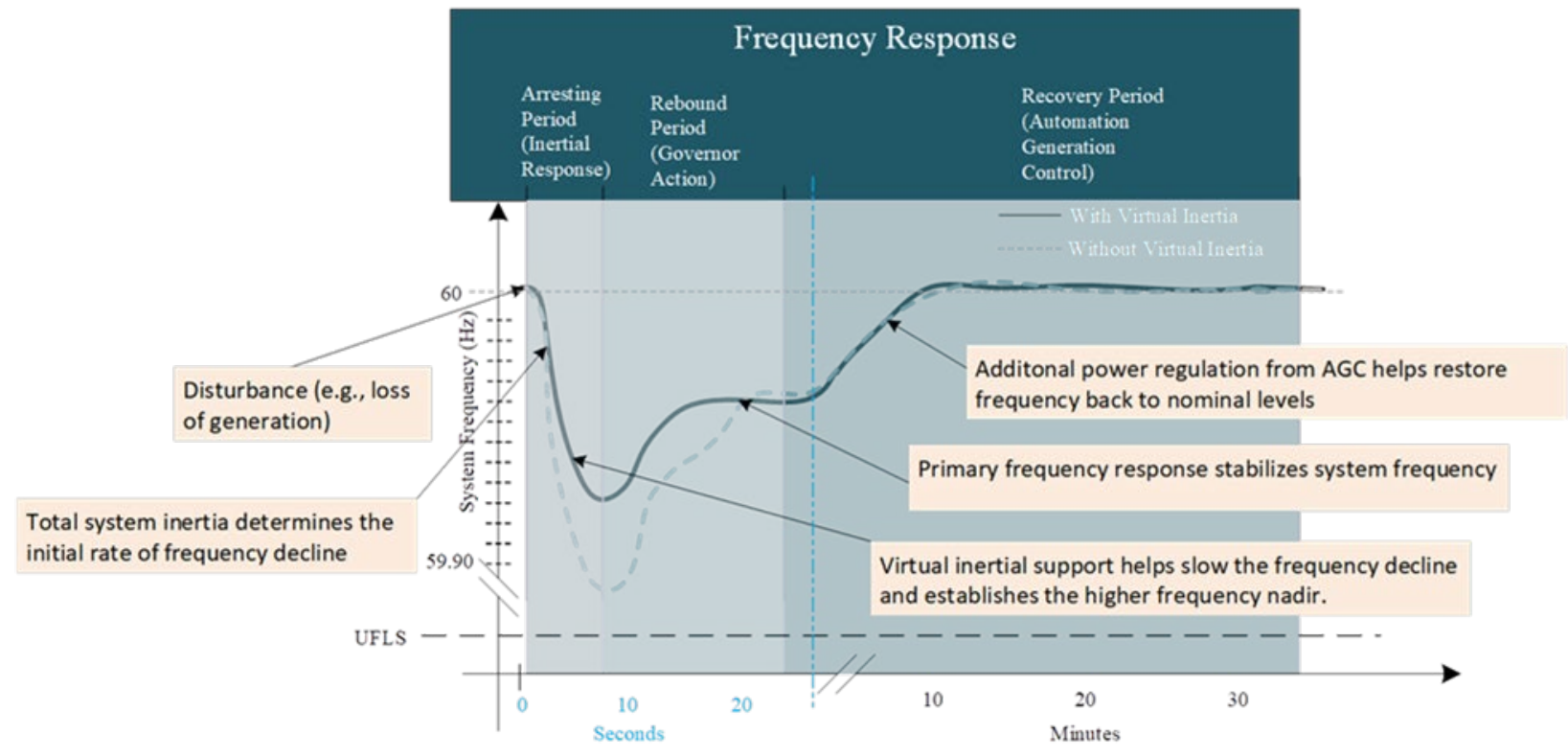

Figure 16 Represenation of a Multiple Time-frame Frequency Response in a Power System Following a Frequency Event.

\subsubsection{Proposed VSG-based Control for DERs}


Without any mechanical rotational part, inverters have a high response speed compared to the conventional rotational machines [30]. Virtual inertia concept is introduced as a solution to overcome this limitation [31]. By emulating the mechanical equation of a real synchronous generator into the inverter, similar behavior can be assumed during normal operation of the system and frequency disturbances; for example, when there is a sudden change (increase or decrease) in active power. Utilizing VSG algorithm, synchronized active power can be injected from the PV to the grid to stabilize the frequency [32], [33]. In this paper, during normal operation of the system (rated frequency and voltage), the perturb and observe method (P\&O) sets the active power reference $P_{\text {ref }}$ by measuring voltage and current of the PV [34]. This active power is controlled in two stages. At the first stage, primary frequency is implemented in the same way as a SG. In the second stage virtual inertia and damping are added to complete the loop. The result is a reference angle that will be fed into park transformation [35]. VSG control can be divided into two sections. First, the mechanical swing equation needs to be emulated and solved numerically. Then the results are used as a reference to control the voltage and current of the inverter. Figure 17 depicts the proposed VSG control in a PV DG.

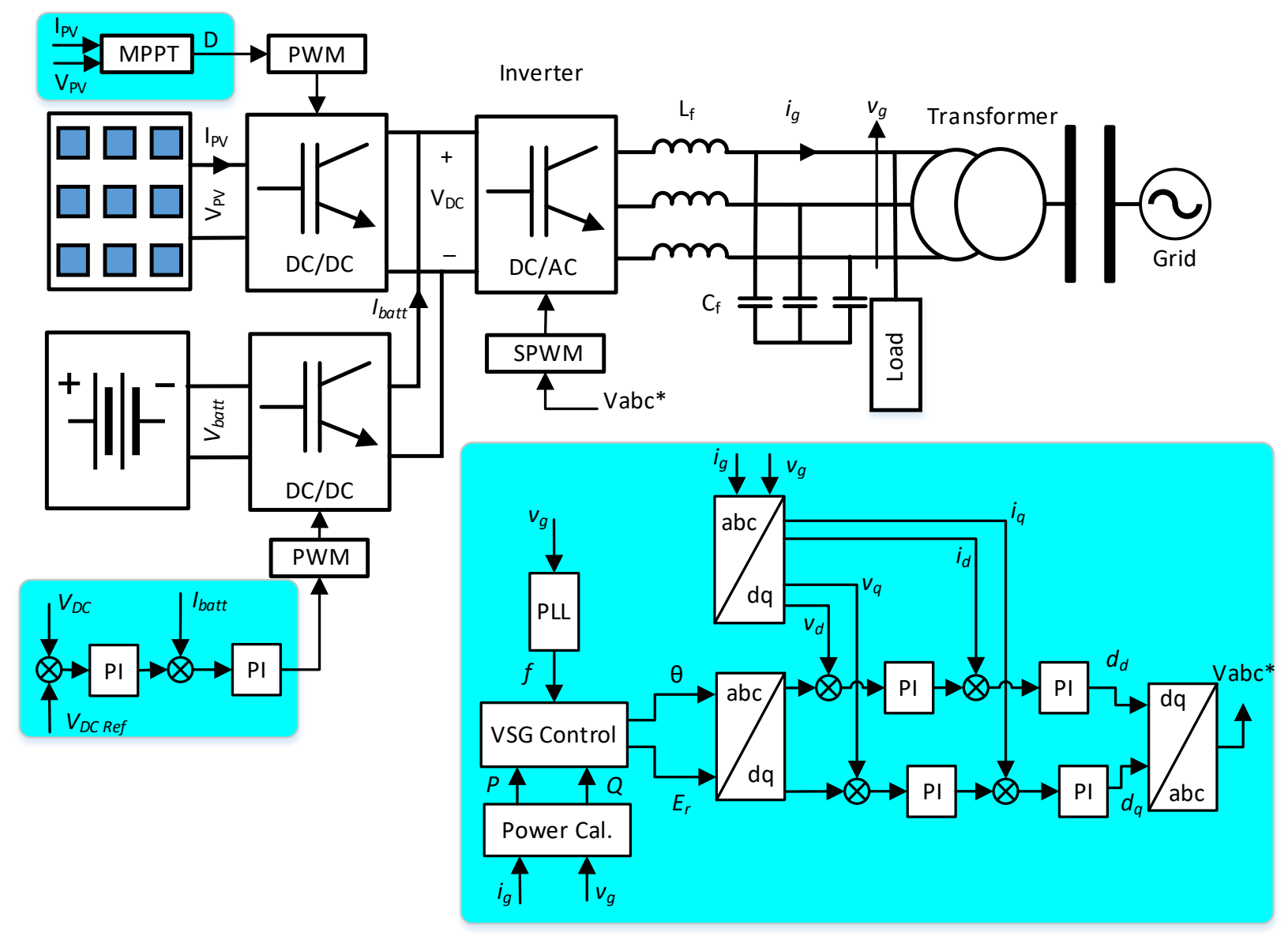

Figure 17 The Proposed VSG-based Multi-Loop Control Method in a PV DG.

Active Power and Frequency (P-f) Control 


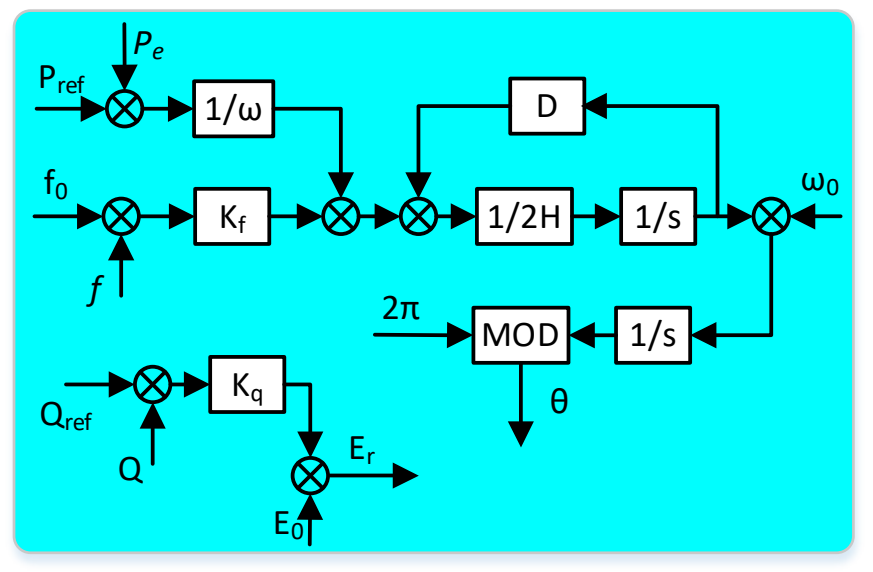

Figure 18 VSG control schematic

The schematic for the VSG control loop is shown in Figure 18. Mechanical equation of a SG assuming that the rotor is a rigid body can be described as

where $H$ is the inertia constant in p.u. derived from

$$
\left\{\begin{array}{c}
\frac{d \theta}{d t}=\omega \\
2 H \frac{d \omega}{d t}=T_{m}-T_{e}-D \Delta \omega
\end{array}\right.
$$

$$
\mathrm{J}=2 \mathrm{H} \frac{S_{\text {base }}}{\omega_{0}^{2}}
$$

where Sbase is the base power of the machine, $\omega$ is the angular frequency of the SG and $\omega_{0}$ is the rated angular frequency [36].

$T_{m}$ and $T_{e}$ are the mechanical output torques of the prime mover and the electromagnetic torque of the SG respectively and can be calculated using:

$$
\left\{\begin{array}{c}
T_{m}=k_{f}\left(f_{0}-f\right)+\frac{P_{r e f}}{\omega} \\
T_{e}=\frac{P_{e}}{\omega}
\end{array}\right.
$$

in which $P_{r e f}$ is the rated active power and $P_{e}$ is the output power of the DG. The primary frequency control method and damping coefficient are working here similar to a real SG and are achieved through a proportional cycle in which $k_{f}$ is the droop coefficient and D is called the damping coefficient. Typically for synchronous machines $H$ varies between 2 and $10 \mathrm{~s}$ [37]. The VSG response at a specific output power and voltage is determined by parameters of its second order differential equation which are the real part of its eigenvalues $\sigma_{i}$ and the damping ratio $\xi_{i}$. These parameters are related to $J$ and $D$ directly through the following equation set:

$$
\sigma_{i}=-\frac{D_{i}}{2 J_{i} \omega_{s}}
$$




$$
\begin{gathered}
\omega_{n i}=\sqrt{\frac{P_{\text {max }} \cos \left(\theta_{i g}\right)}{J_{i} \omega_{s}}} \\
\xi_{i}=-\frac{\sigma_{i}}{\omega_{n i}}
\end{gathered}
$$

where $P_{\operatorname{maxi}}$ is the maximum transferable power from the VSG bus to the grid, $\theta_{i g}$ is the voltage angle of the VSG with respect to the grid and $\omega_{n i}$ is the undamped natural frequency of the VSG. At any working conditions, the parameters corresponding to the desired system response can be achieved by tuning $J$ and $D[38]$.

\section{Reactive Power and Voltage (Q-E) Control}

Controlling the voltage is achieved by regulating the reactive power as follows:

$$
E_{r}=E_{0}+k_{q}\left(Q_{r e f}-Q\right)
$$

where $E_{r}$ is the reference voltage, $k q$ is reactive power droop coefficient and $E_{0}$ is the nominated voltage amplitude.

\section{Inner Voltage and Current Control Loops}

This control loop features a conventional outer voltage and inner current loop. Its primary function is to regulate the output voltage with no steady state error while quickening the dynamic response of the current loop to strengthen the ability of inverter control. This can be achieved through the outer voltage and inner current control loop. The calculated reference voltage $E_{r}$ is set as the reference for the outer voltage loop. Since the control is achieved in constant reference frame, $E_{r}$ is transformed from synchronous to dq reference frame using Park transformation with the calculated angle in VSG control as the input angle for the transformation.

$$
E_{r}=\left[\begin{array}{c}
E_{a r} \\
E_{b r} \\
E_{c r}
\end{array}\right]=\left[\begin{array}{c}
E \sin (\omega t) \\
E \sin \left(\omega t-\frac{2}{3} \pi\right) \\
E \sin \left(\omega t+\frac{2}{3} \pi\right)
\end{array}\right]
$$

Four PI (proportional integral) controllers are then used to regulate the $d-q$ axis terminal voltage and current, respectively. The controller will guarantee stable steady-state and dynamic performance. The output of the controller will be the modulation signals that will be sent to the pulse-width- modulated (PWM) generator to drive the inverter's switches.

\subsubsection{Impact of DERs with and without VSG on BES}

Figure 19 shows the sample power grid with generation, transmission and distribution simulated in MATLAB/Simulink. The Kundur's two-Area system with parameters taken from [28] which is comprised of four synchronous generators (two in each area) that are boosting up with transformers and connected through transmission lines. The areas are connected to each other through a tie line. IEEE 13 node test feeder [29] is used as distribution system. The DGs 
such as PV and battery energy storage unit (BES) are connected to distribution system using a voltage source inverter (VSI). PV system includes PV arrays and a unidirectional boost DC-DC converter which is working under perturb and observe $(\mathrm{P} \& \mathrm{O})$ maximum power point tracking (MPPT) control. BES includes batteries and a bidirectional boost DC-DC converter controlled by multi-loop voltage and current control. The outer loop controls voltage and inner loop controls current through proportional integral (PI) controllers. The BES and PV unit are connected in parallel and form the DC link.

This model was used to study different cases such as normal operation and under sudden changes in loads to investigate the impact of DERs with VSG on the system frequency regulation. The DC output voltage of the PV system is $30 \mathrm{kV}$, and the AC voltage regulated by the inverter is $13 \mathrm{kV}_{\text {L-Lrms. }}$. The controller of the VSG is described in Section 3.1.2.

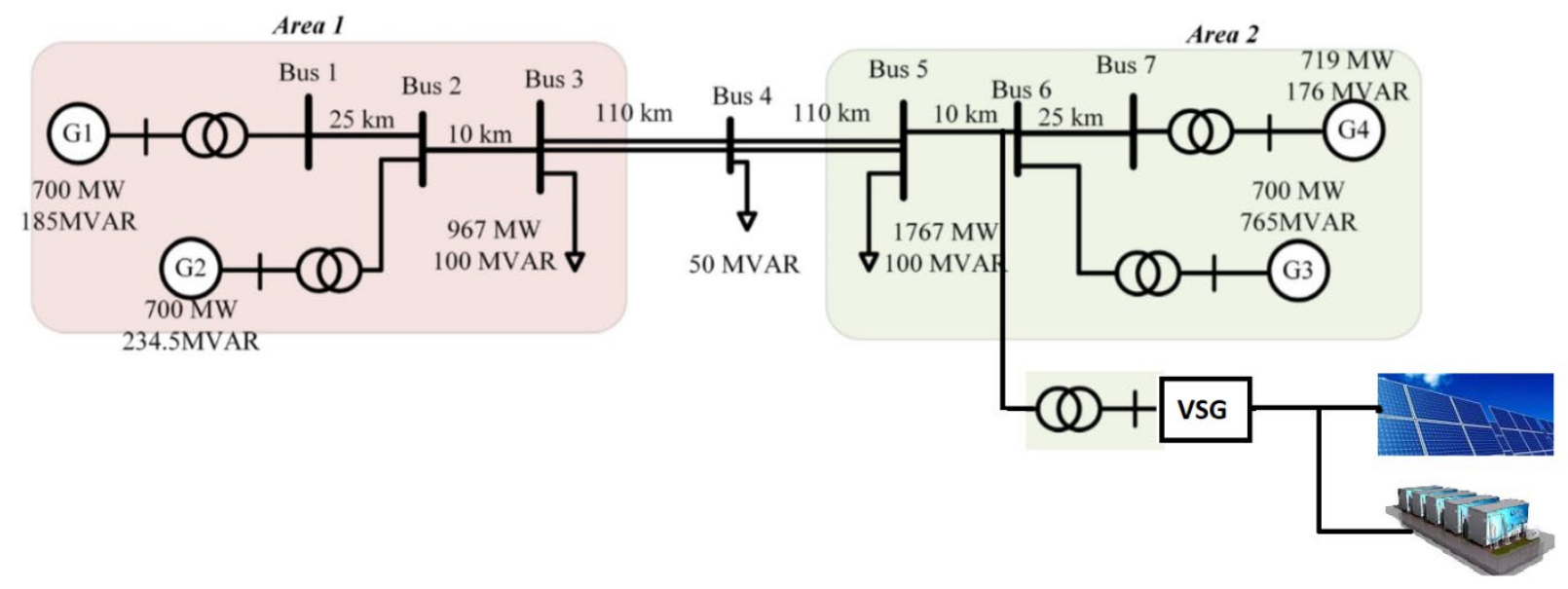

Figure 19 T\&D Combined System with VSG Technologies.

First, multiple simulations are performed where the inertia of generator 3 (G3) in Area 2 is reduced without adding any DERs into the system. In each simulation, a sudden load increase in Area 1 is introduced at $25 \mathrm{~s}$. The system frequency is captured in Figure 20, which shows that the frequency nadir is increased with decreasing the inertia. This result corresponds to the effect of adding DERs to the system and replacing the synchronous generators. 


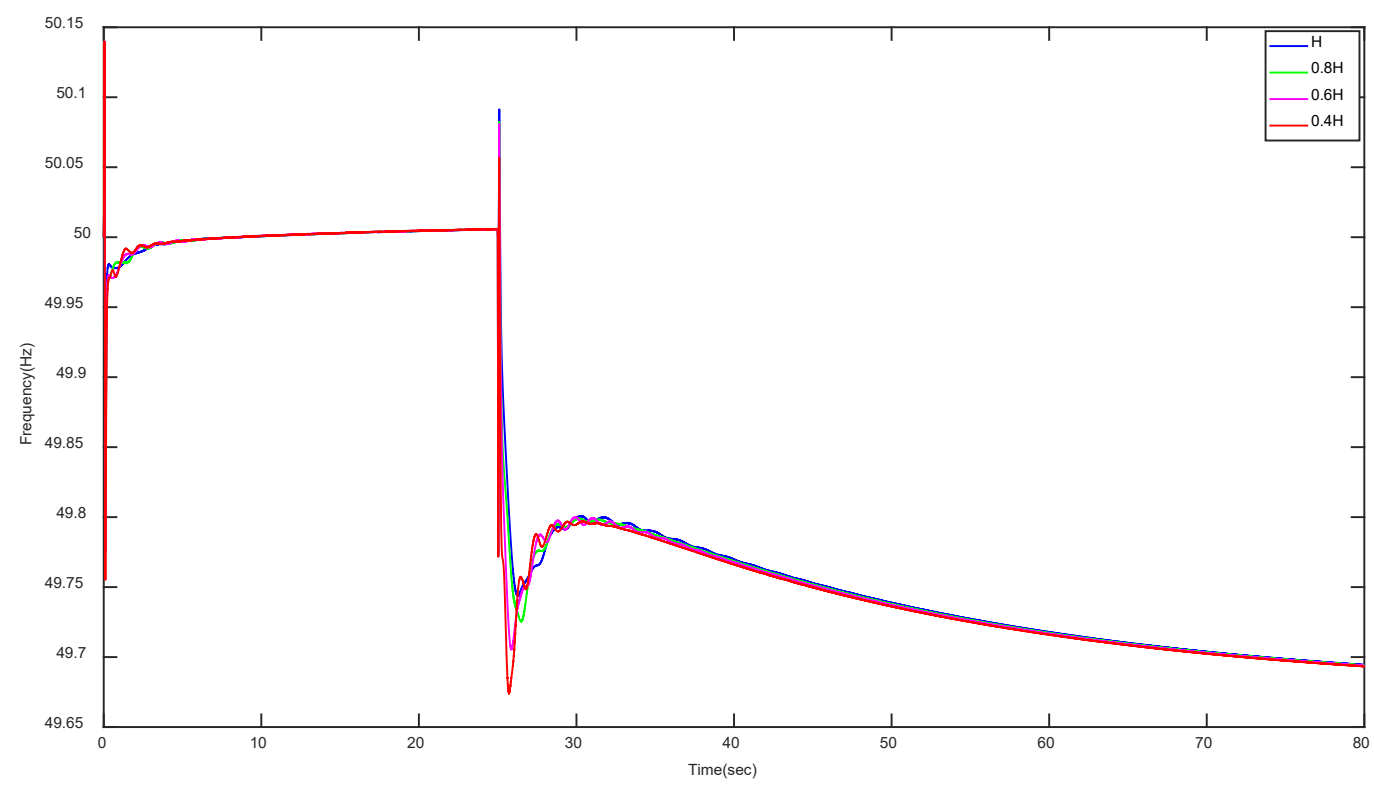

Figure 20 System Frequency with Different Inertia in SGs.

The next two sets of simulations are conducted to study the effect of DERs with and without VSG on the BES. For the multiple cases simulated, the total simulation time is 80 seconds, and a sudden load increase of $160 \mathrm{MW}$ in Area 1 is introduced at $25 \mathrm{~s}$. In Figure 21, the system frequency performance is compared for three scenarios: (1) with no DERs, (2) with state-of-the-art power electronics inverters that do not emulate inertia, and (3) with DERs equipped with VSG with an emulated inertia effect. The DERs are rated at $200 \mathrm{MW}$. Figure 22 compares the system frequency performance for the same three scenarios, and the only difference is that the DERs are rated at $400 \mathrm{MW}$. The results in Figure 21 and Figure 22 show that the frequency nadir decreases when DERs without virtual inertia are integrated, and the frequency nadir increases when DERs with virtual inertia are integrated. 


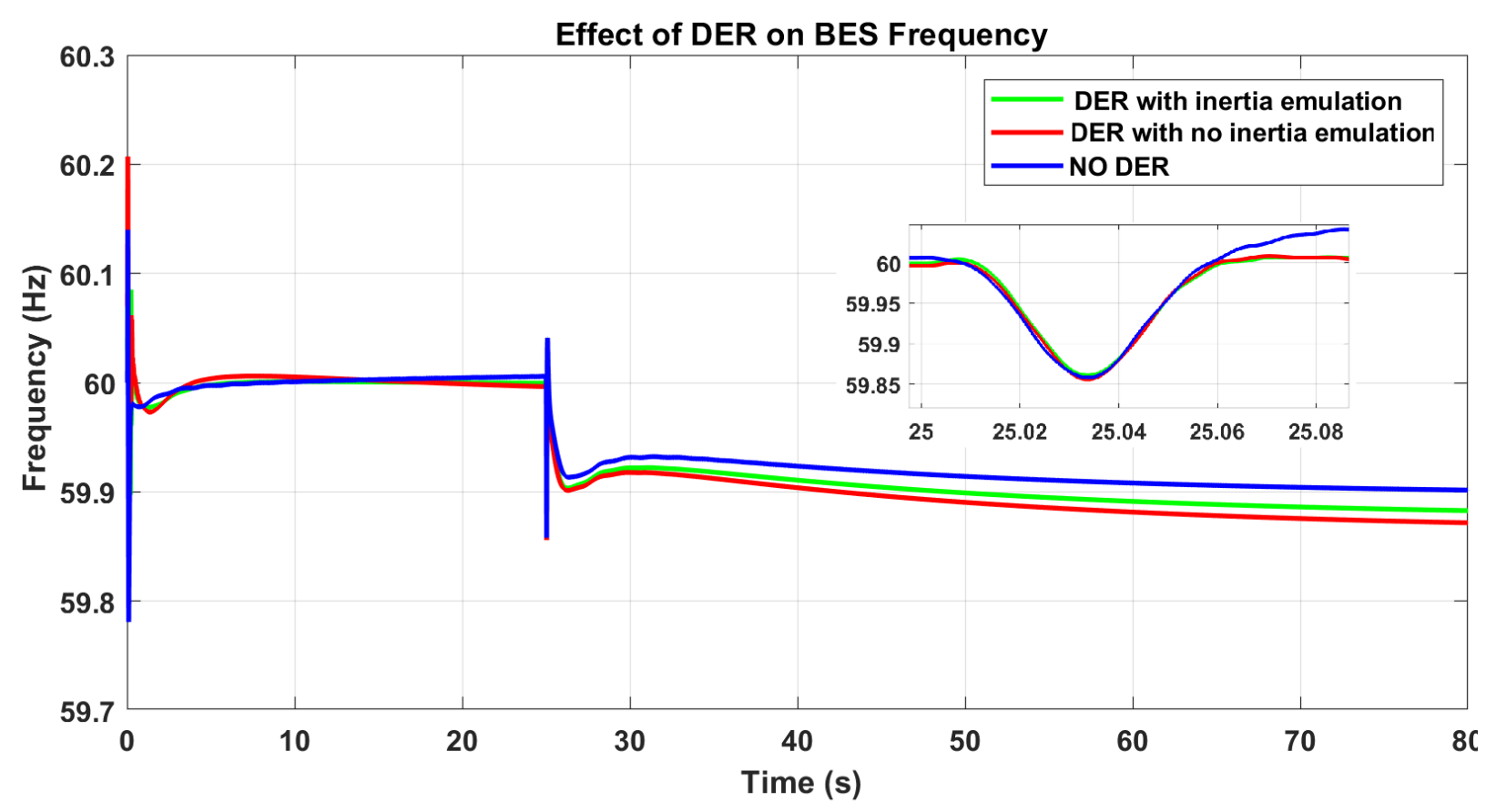

Figure 21 System Frequency with No DERs, with DERs but No Inertia Emulation, and with DERs and Inertia Emulation, respectively, at $200 \mathrm{MW}$.

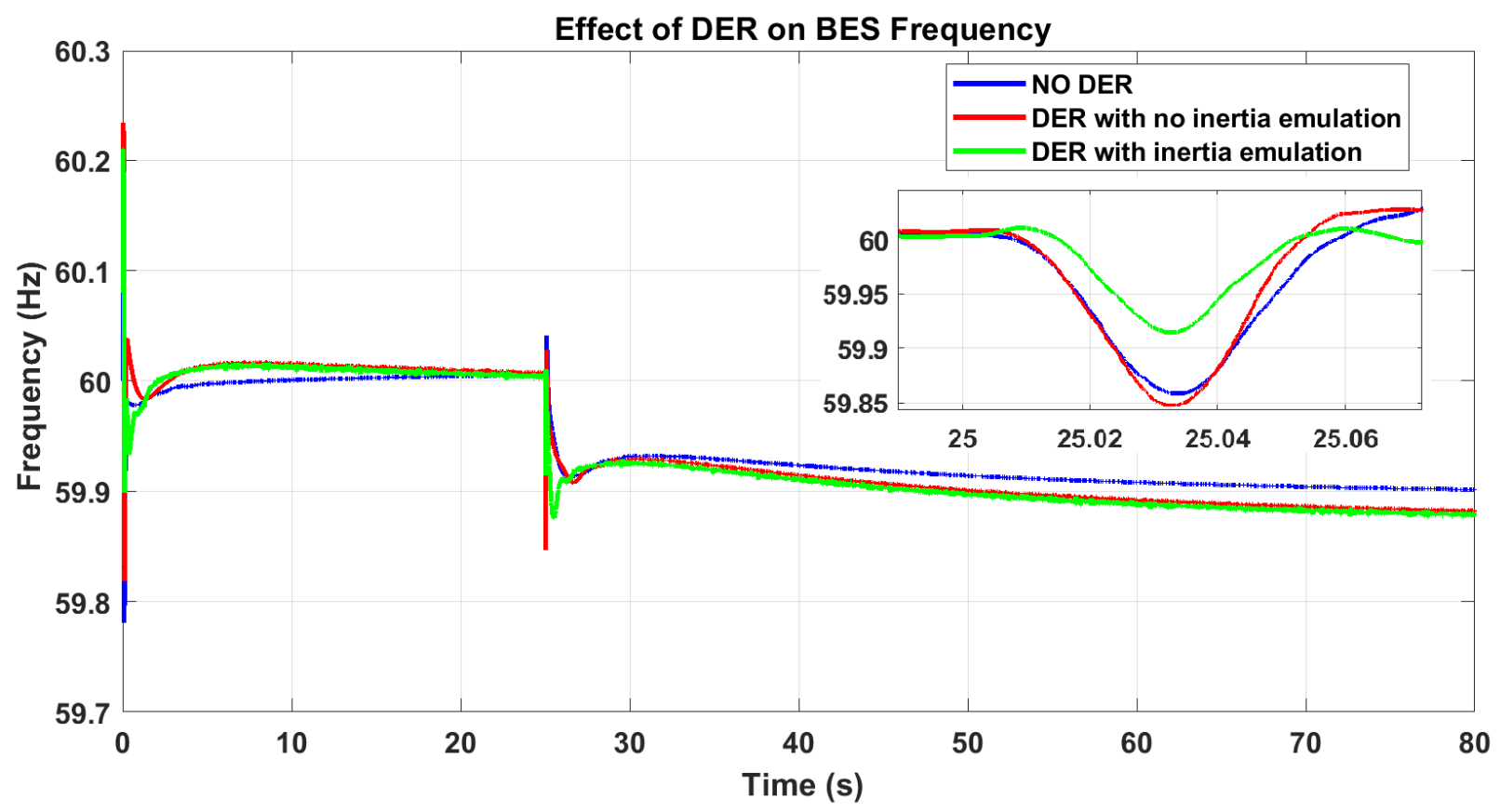

Figure 22 System Frequency with No DERs, with DERs but No Inertia Emulation, and with DERs and Inertia Emulation, respectively, at $400 \mathrm{MW}$. 
In addition, four sets of simulations are conducted in which the DERs penetration level in Area 2 is increased from $0 \mathrm{MW}$, to $200 \mathrm{MW}, 400 \mathrm{MW}$, and finally $600 \mathrm{MW}$. For each DERs penetration level, the SGs' capacity and inertia in Area 2 (SG \#3) is reduced accordingly to simulate the effect of DERs displacing SGs and how the virtual synchronous generator will emulate the inertia lost by decreasing the SGs' capacity. Throughout all four sets of simulations, the configuration of Area 1 is kept constant, i.e., without any DER penetration. The system frequency response corresponding to the different DER penetration levels is depicted in Figure 23, which shows that the VSG is emulating the generator inertia; and therefore, even with a large increase in the DER penetration level, the frequency nadir is almost the same as with the generators only.

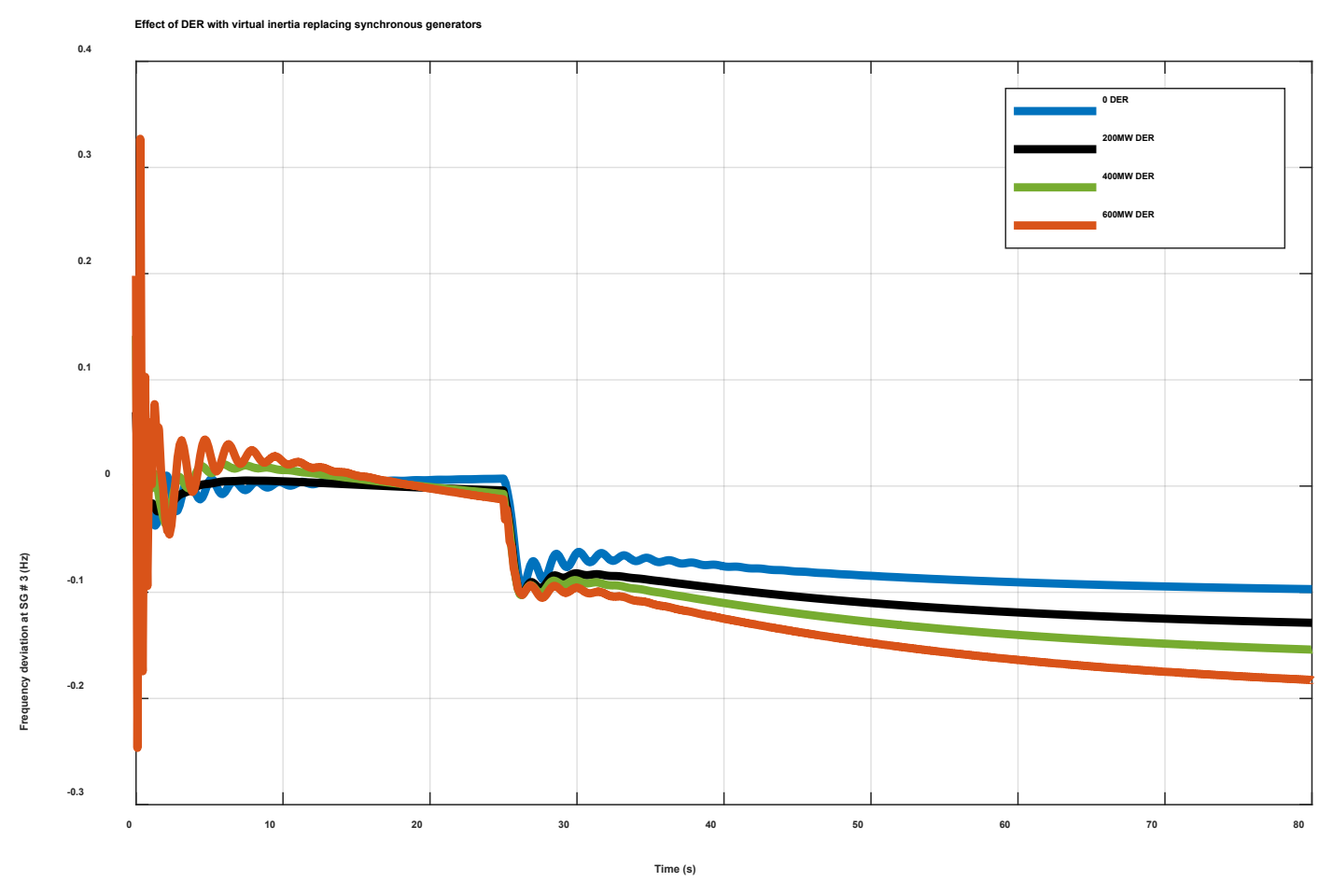

Figure 23 Effect of DER with VSG on BES Frequency.

\subsection{DERS' IMPACT ON BES NET LOAD VARIABILITY AND RAMPING AND MITIGATION SCHEMES}

Local and state governments in the United States have mandated, through renewable portfolio standards (RPSs), that a fixed percentage of electricity produced by utilities serving in the area must be generated through renewable energy resources. This mandate has subsequently resulted in a proliferation of grid-connected DERs. The extensive body of literature on the impact of the large-scale integration of DERs suggests that the unplanned interconnection of large numbers of DERs can have an adverse impact on the power grid. Adverse impacts that can result could be in terms of controllability, interoperability, and visibility of the load in the system. As DERs' power output can be variable, extra levels of precaution are required in the 
operation of the power grid. One of the major issues that can become evident during power grid operation with high penetration of solar-based PV systems is the inability to meet the ramp rate of demand in the system during the evening as PV power output drops.

Consider a power grid with a large penetration of PV systems: in such systems, as the sun sets and generation from the PVs reduces, the conventional generators must pick up the slack. Such a scenario can be worsened when the load demand in the system starts to increase in the evening hours, driving an even higher rate of change of generation from the conventional generators. However, there is an intrinsic limit on the rate of change of generation possible with conventional generators. Thus, an unplanned, large-scale penetration of renewable energy resources can be detrimental to the operation of the power grid because of their effects on the system net load.

\subsubsection{Case Study on the CAISO System}

The "net load" of a distribution system is defined as the actual load minus the power output from variable generation sources (i.e., DERs such as wind, PV, etc.) connected to the distribution system. Understanding the nature of net load is crucial as it represents the portion of the load that is to be served by the conventional generator sources. Studies performed by the California Independent System Operator (CAISO) found that the increased penetration of renewable energy resources in the power grid caused the net load observed in the system to drop during the day and quickly ramp up to a late-day peak. Furthermore, as the penetration has grown during the period of study, the drop and ramp-up of net load was observed to be more pronounced (i.e., more stressful on the distribution system). This net load pattern resulted in the "duck curve" chart shown in Figure 24.

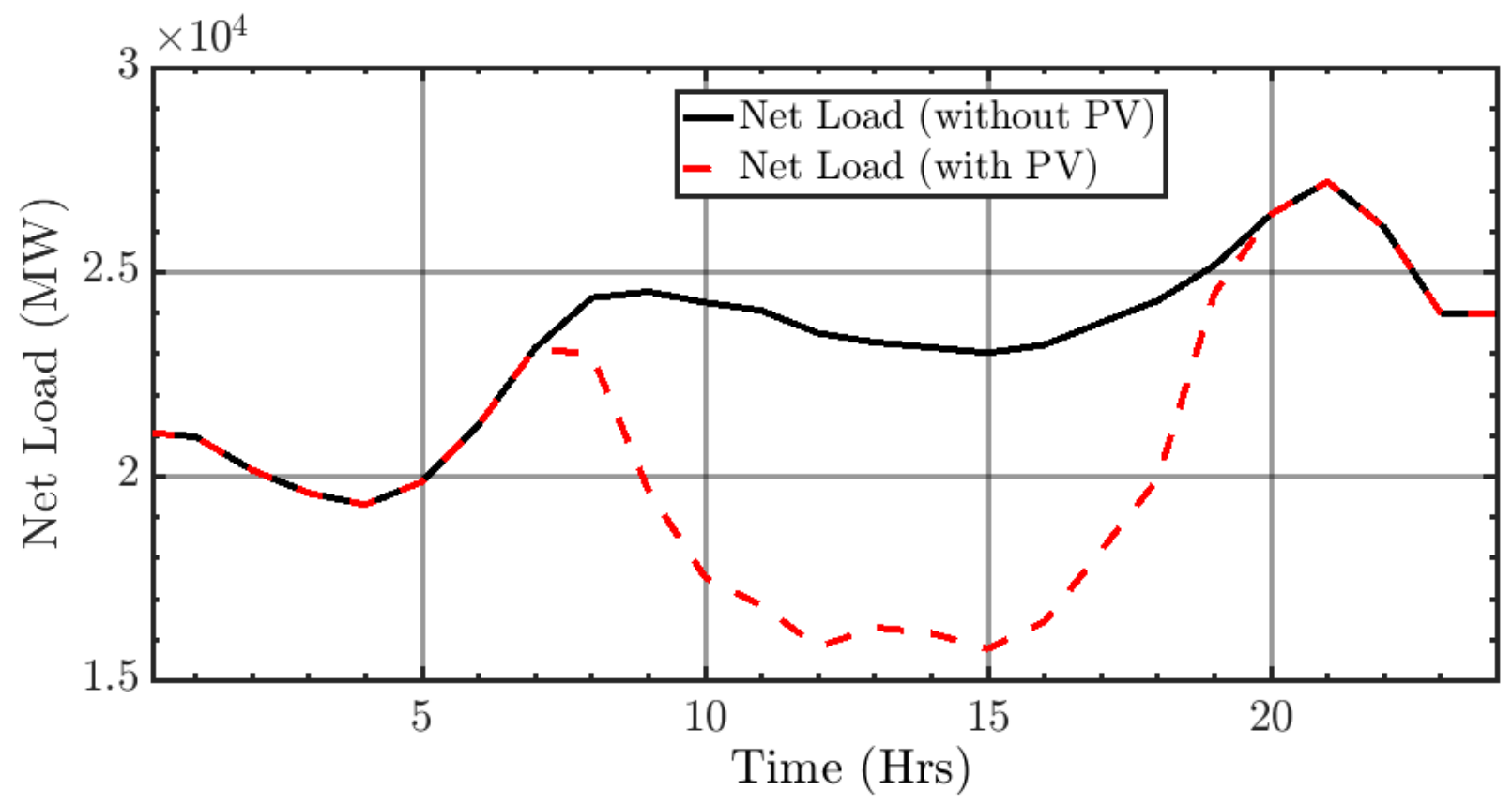

Figure 24 Average Net Load in the CAISO Studies (in red) and the Actual Load (black) in the System. 
A recent study [28] discusses that the cause of the duck curve in terms of system load is attributable not only to DERs but also to utility-scale solar farms. In addition, it is prudent to understand the underlying cause of the duck curve in the system before setting out to develop a solution, given that the solution for DERs may differ from that for the utility-scale PV system. An analysis of system load (i.e., total load in the system minus the load served by behind-themeter systems) shows that from January 2011 to June 2016 in [28] for California, the system load is essentially unchanged - indicating that the duck curve behavior evident in the net load could be caused by the utility-scale PV systems.

CAISO provides historical energy management system (EMS) hourly load data and PV generation data through its website [40]-[41], which has been utilized in this case study to demonstrate the load ramping impact caused by PV penetration.

Figure 24 shows the net load observed by CAISO for two different cases - with and without PV generation. It is evident that with PV generation, the net load in the system drops during the day owing to the power supplied from PV generation and ramps up at a faster rate during the evening as the sunset coincides with a period of increased load demand. On a particular day - May 1, 2018 - the net load ramp rate increases from $676 \mathrm{MW} / \mathrm{hr}$ to $2,122 \mathrm{MW} / \mathrm{hr}$ because of the PV generation for the duration between $3 \mathrm{pm}$ to $8 \mathrm{pm}$. The PV generation for this day is shown in Figure 25.

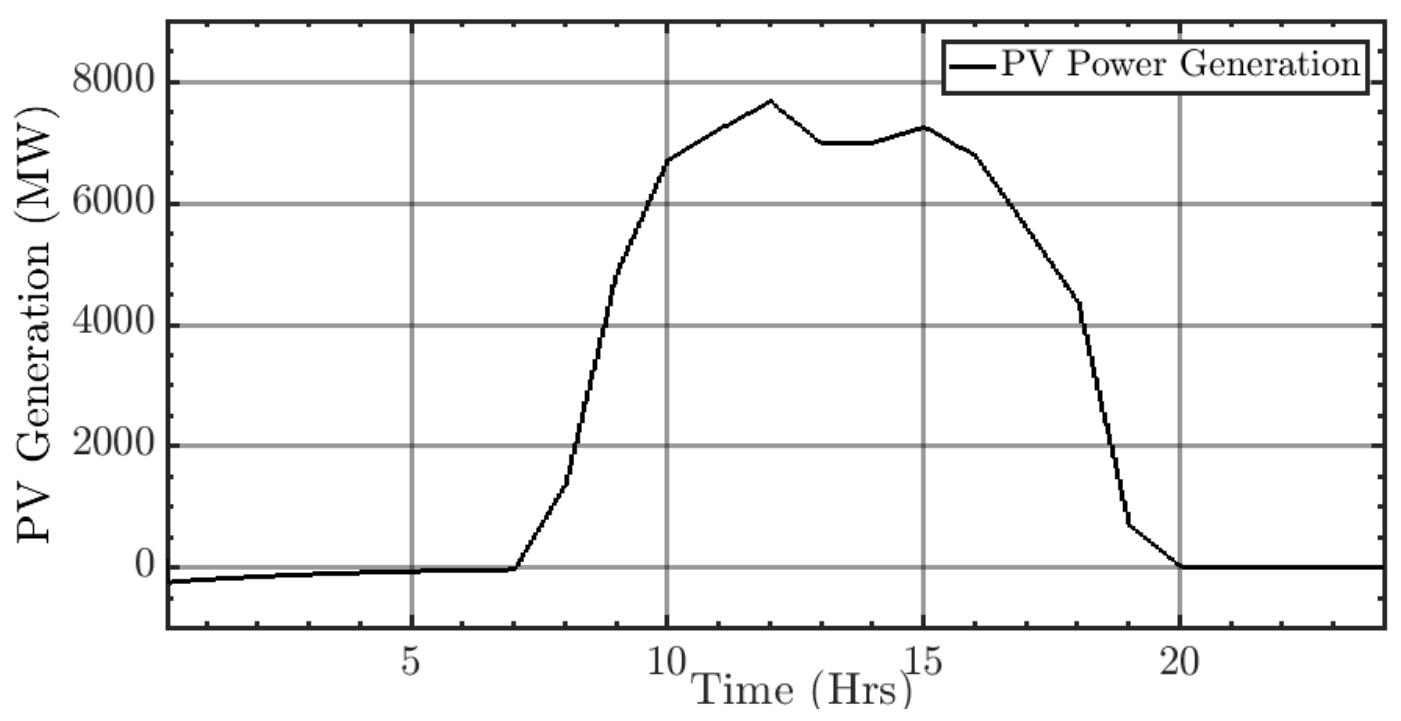

Figure 25 PV Power Generation in the CAISO Area on May 1, 2018.

To address the challenges brought by the steep demand ramping, two possible solutions have been discussed in the industry, as follows:

a) Flatten the duck curve: This approach essentially means consuming excess generation during the day or curtailing solar generation during the day to prevent the decrease in net 
load from morning to early evening hours. If the consumer loads can be shifted from evening to the daytime when the solar output is higher, then the demand during the evening would not rise as quickly and as high as in the scenario without any load management. Utilities are now offering new pricing mechanisms based on the time of the day to encourage consumers to use electricity during the day when solar is more abundant. The other solution that has been given consideration is the curtailment of PV during the daytime. If PV output is curtailed during the daytime, then the drop in net load during the daytime would not be so low to create a steep evening ramp.

b) Fatten the duck curve: This approach means adding new but more dispatchable resources into the power grid, such as through the use of electric vehicles and energy storage systems. For example, if battery energy storage systems (BESSs) are added to the grid, they can be charged during the day when PV generation is higher and can be discharged in the evening to reduce the steep evening ramp as the sun goes down. However, BESSs may be too cost prohibitive to be widely deployed.

Recently, BESSs have emerged as a promising solution to this problem. By utilizing BESSs, adverse impacts caused by the sudden fluctuations in PV generation can be negated, thus leading to a more reliable and stable grid. Figure 26 demonstrates how a BESS can be used to minimize the load ramp rate caused by fluctuations in PV generation.

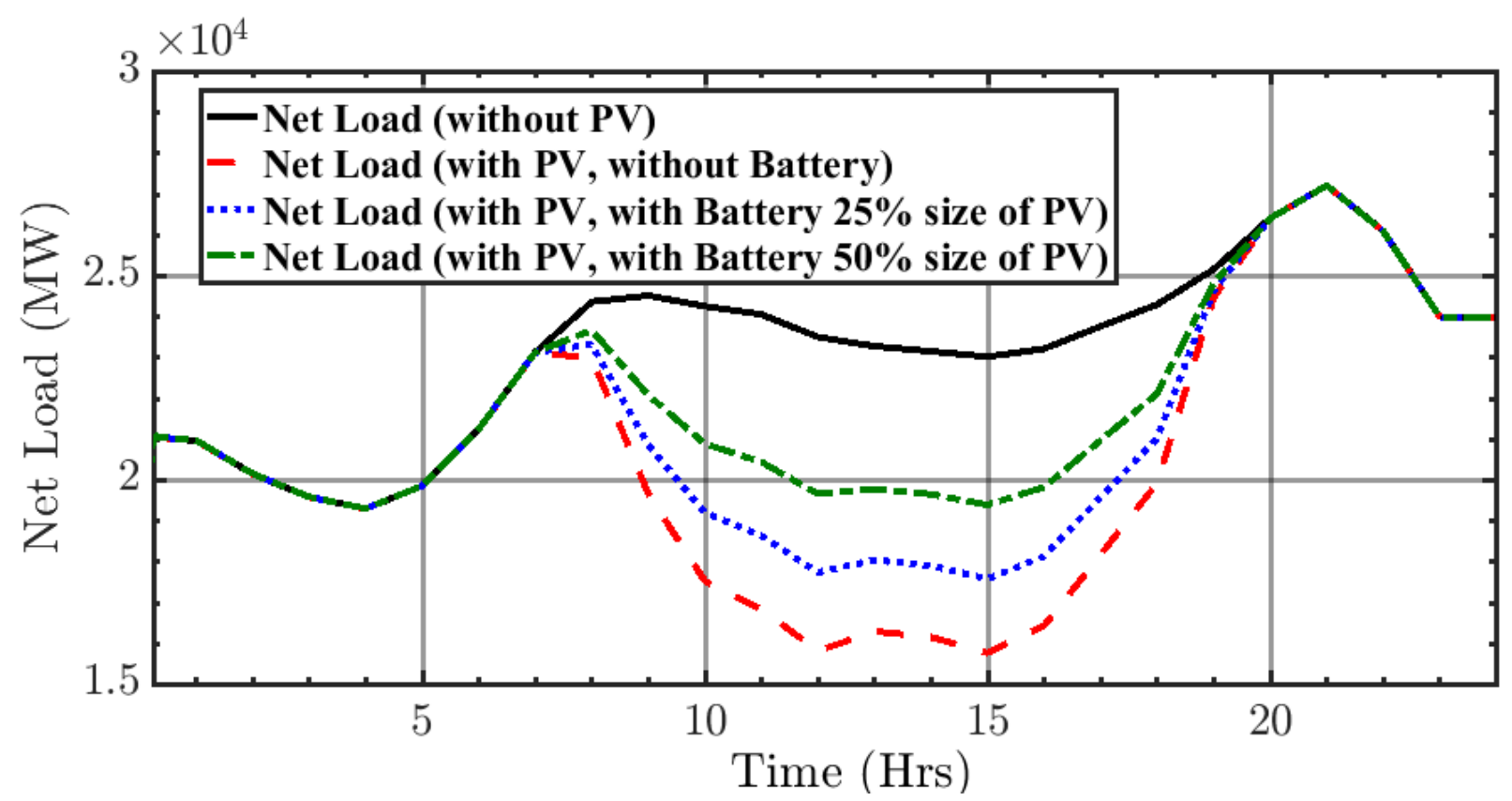

Figure 26 Net Load in the CAISO Studies with and without PVs and with the Use of the BESS.

In this case study, a simple approach is used in which the BESS stores the excess power generated by PVs. Please note that for this demonstration, the BESS is not discharged in the 
evening to lower the net load. Improved net load smoothing can be achieved through various techniques and will be addressed in Section 3.2.2.

\subsubsection{Case Study on Kundur Two-Area System}

In this section, the 5-minute sampled load profile from CAISO is scaled to appropriately fit the Kundur Two-Area system such that during the maximum load demand in the system without any DERs, Area 1 exports power equal to $420 \mathrm{MW}$ to Area 2. The aggregated loads in both Areas 1 and 2 are replaced by a dynamic load, where the load profile obtained from CAISO is shared proportionally between the loads in two areas in the same ratio as in the base case. Similarly, PV power output obtained from CAISO is also scaled proportionally to represent various penetration levels of PVs in the system. The total PV power output is divided equally in both areas, such that for a total PV power output of $500 \mathrm{MW}$, each area contributes $250 \mathrm{MW}$. Four different sets of simulations are performed to demonstrate the "duck-curve" phenomena in the system. Figure 27 shows a comparison of the net load in the system for the base case without PVs and for three cases when the maximum PV power output is $250 \mathrm{MW}, 500 \mathrm{MW}$, and $800 \mathrm{MW}$, corresponding to $8 \%, 18 \%$, and $29 \%$ penetration levels, respectively. Figure 27 shows that as the PV penetration increases and the evening load ramp-up coincides with the drop in PV power output after sunset, the slope in net load demand during the evening hours becomes steeper. This outcome has a direct impact on the frequency regulation capability of the system as the conventional generation sources may not be able to follow this steep change in the load demand. Table 6 reports a comparison of net load ramping rates observed on evening hours from $5 \mathrm{pm}$ to $8 \mathrm{pm}$ for different levels of PV penetration.

Table 6 Comparison of Net Load Ramping Rates at Different PV Power Output Levels.

\begin{tabular}{lcccc}
\hline PV Power Output & $0 \mathrm{MW}$ & $250 \mathrm{MW}$ & $500 \mathrm{MW}$ & $800 \mathrm{MW}$ \\
3-hour (5 pm to 8 pm) Net Load Ramping (MW/hr) & 43 & 118 & 192 & 267 \\
\hline
\end{tabular}

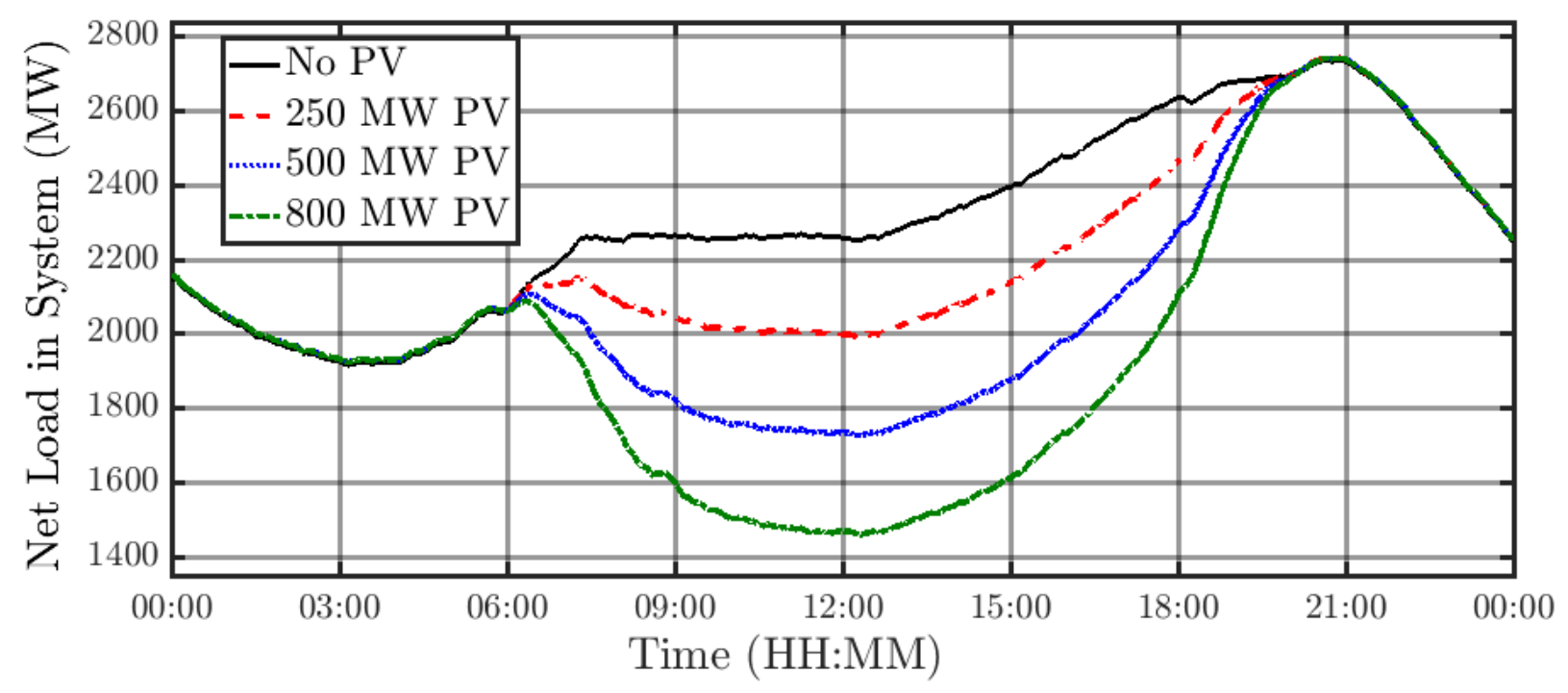


Figure 27 Net Load as Seen on the Kundur Two-Area System with and without PVs.

To mitigate the impact of large penetration levels of PV systems on the BESS, as discussed before, there can be two approaches. Here, we adopt the approach that involves the use of a BESS. The BESS will counterbalance the negative impact that the PV has on the net load: when PV generation increases, the BESS can act as a load; and when PV generation starts to ramp down, the BESS can act as a source. Figure 28 shows how the use of the BESS can minimize the net load ramp-up for the case with $800 \mathrm{MW}$ of PV power output.

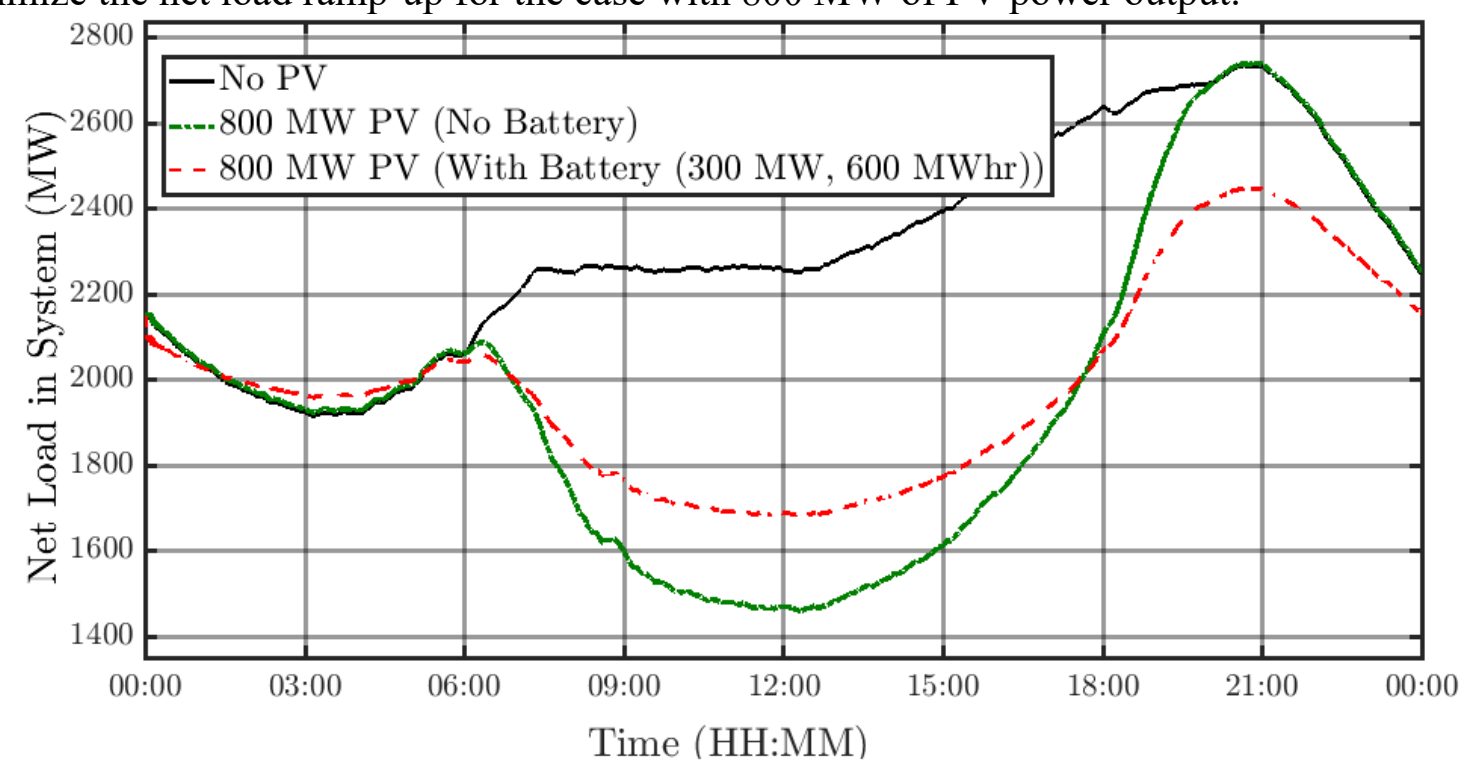

Figure 28 Comparison of the Net Load Profile with no PV, with PV and no BESS, and with both PV and BESS.

Through the use of the BESS, the evening's steep slope in the net load curve can be reduced. In this case, the 3-hour ramp rate of net load is reduced to $159 \mathrm{MW} / \mathrm{hr}$ from 267 $\mathrm{MW} / \mathrm{hr}$, which considerably reduces the ramping requirements on the conventional generation sources. Figure 29 shows a flowchart of a simple management scheme implemented to minimize the net load ramp rate using the BESS. The scheme monitors the power flow at the substation bus and sends data every 5 mins to the smoothing algorithm. The algorithm then compares the substation load data with the desired load flow reference. Based on the difference between the reference and actual substation load flow, BESSs downstream of the substation bus are requested to charge or discharge to bring the actual load flowing from the substation to the reference level. 


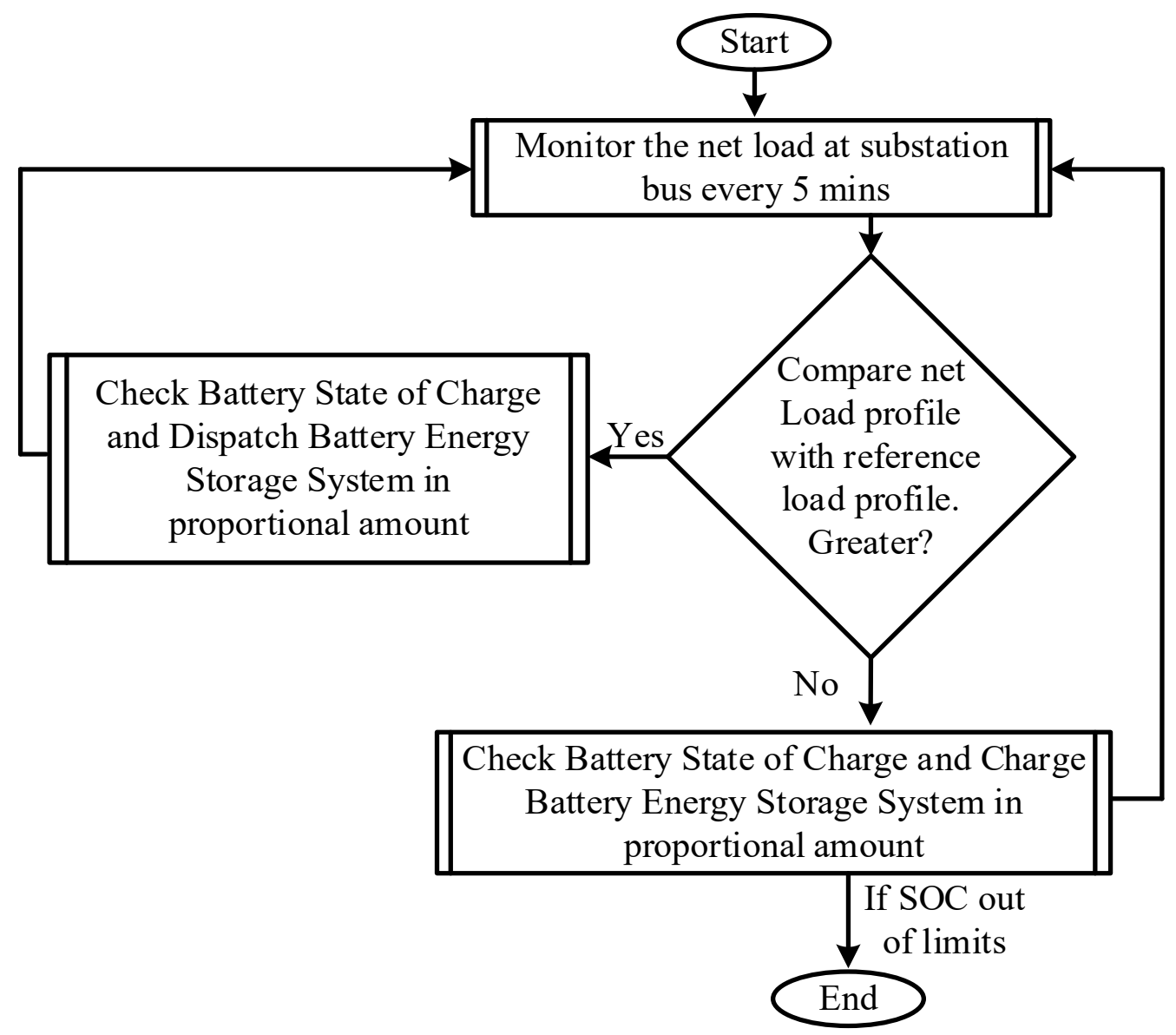

Figure 29 Flowchart of the Implemented Algorithm for Minimizing the Net Load Ramping Rate in the BES. 
This page intentionally blank. 


\section{DERs' IMPACT ON AND CONTROL STRATEGIES TO IMPROVE THE VOLTAGE STABILITY OF THE BES}

This chapter examines DERs' impact on - and the control strategies to improve - the voltage stability of the BES. Using the T\&D combined model, this chapter first explores how the combination of various slopes and time delays of DER VVC curves can affect BES voltage control during normal operations. The impact of interactions between multiple DERs with VVC enabled on the BES is also examined through case studies. This chapter further presents case studies demonstrating that dynamic DER reactive power support can help maintain BES voltages during abnormal conditions.

\subsection{IMPACT OF DER VVC ON BES VOLTAGE DURING NORMAL OPERATIONS}

\subsubsection{Comparison of Performance of Line Regulators and Reactive Power Support from DERs on Voltage Regulation of the BES}

The objective of this section is to compare the DERs' reactive power support capability with line regulators to support BES voltages. To facilitate the studies, three scenarios with various combinations of availability of DER VVC and line regulators are created, as shown in Table 7.

Table 7 Combination of Various Availabilities of DER VVC and Line Regulators.

\begin{tabular}{lcc}
\hline & DER VVC & Line Regulators \\
\hline Case 1 & Disabled & Disabled \\
Case 2 & Enabled & Disabled \\
Case 3 & Disabled & Enabled \\
\hline
\end{tabular}

Figure 2 shows the location of DERs and line regulators in the distribution feeder. The settings of the line regulators are shown in Table 8. Figure 30 illustrates the VVC curve used in DERs; the corresponding settings of the Volt-VAR curve are as follows:

$$
\mathrm{V}_{\mathrm{L}}=0.50 \text { p.u., } \mathrm{V}_{1}=0.90 \text { p.u., } \mathrm{V}_{2}=1.00 \text { p.u., } \mathrm{V}_{3}=1.00 \text { p.u., } \mathrm{V}_{4}=1.10 \text { p.u., } \mathrm{V}_{\mathrm{H}}=1.2 \text { p.u. }
$$

IEEE Standard 1547-2018 suggests these parameters as default values for the Volt-VAR curve implementation for Category A DERs. Category A requires DERs to have the minimum performance capabilities needed for Area EPS voltage regulation, and almost all of the state-ofthe-art DERs can attain these requirements. The volt-VAR control for DER is enabled at time $\mathrm{t}=10$ seconds for this case study. 
Table 8 Operational Settings Used for Line Regulators.

\begin{tabular}{ll}
\hline Line Regulator 1 Settings & \\
\hline Tap Selection time & 4 seconds \\
Reference Voltage & 1.02 p.u. \\
Dead band & 0.03 p.u \\
Tap change delay & 1 second \\
\hline Line Regulator 2 Settings & \\
\hline Tap Selection time & 4 seconds \\
Reference Voltage & 1.03 p.u. \\
Dead band & 0.03 p.u \\
Tap change delay & 1 second \\
\hline
\end{tabular}

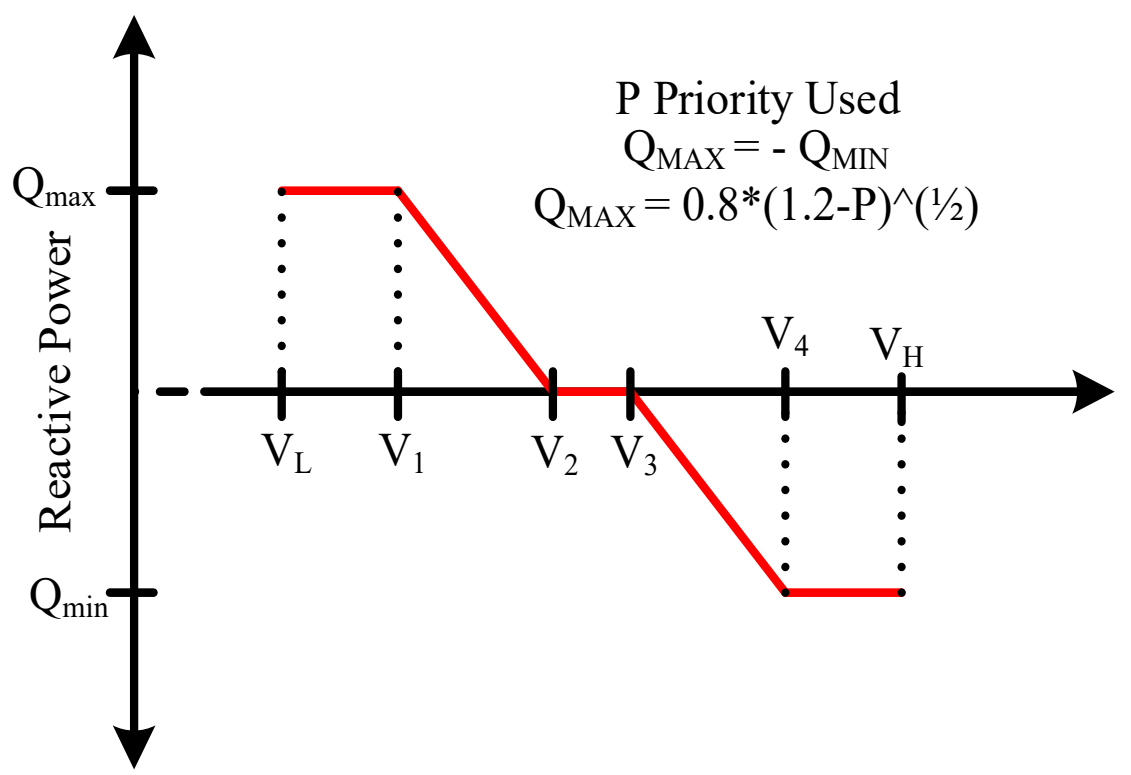

Figure 30 DER Volt-VAR Curve Illustration.

The test system is subjected to a 75-MW load increment at 20 secs, 30 secs, and 40 secs in bus 9 of Area 2. The operational conditions for the T\&D systems for the case studies are reported in Table B-1.

Figure 31 shows the comparison of the voltage profiles following the load changes in Area 2 at the sending-end and receiving-end of the tie-line for three cases. The first is the base case or Case 1 (black solid line) without any form of voltage regulation from line regulators or of reactive power support of DERs. In the second case or Case 2 (red dotted line), DER VVC is enabled and line regulators are disabled. In the third case or Case 3 (blue dotted line), line regulators are enabled and DER VVC is disabled. The receiving-end voltage for the case with only voltage support from line regulators is the lowest, which demonstrates the possible BES 
voltage instability that can be caused by the distribution system's line regulator operation. The line regulators maintain the voltage within the distribution system close to the nominal value but do not inject reactive power into the system, thus causing the voltage-dependent, constantimpedance types of loads to draw the reactive as well as active power they need from the BES. With more power drawn from the BES by the distribution system, the voltage on the receiving end of the tie-line voltage falls to a lower value. If the load continues to increase and the line regulator operates to maintain the voltage within the distribution system, the BES voltage may continue to fall, and the system may become unstable.

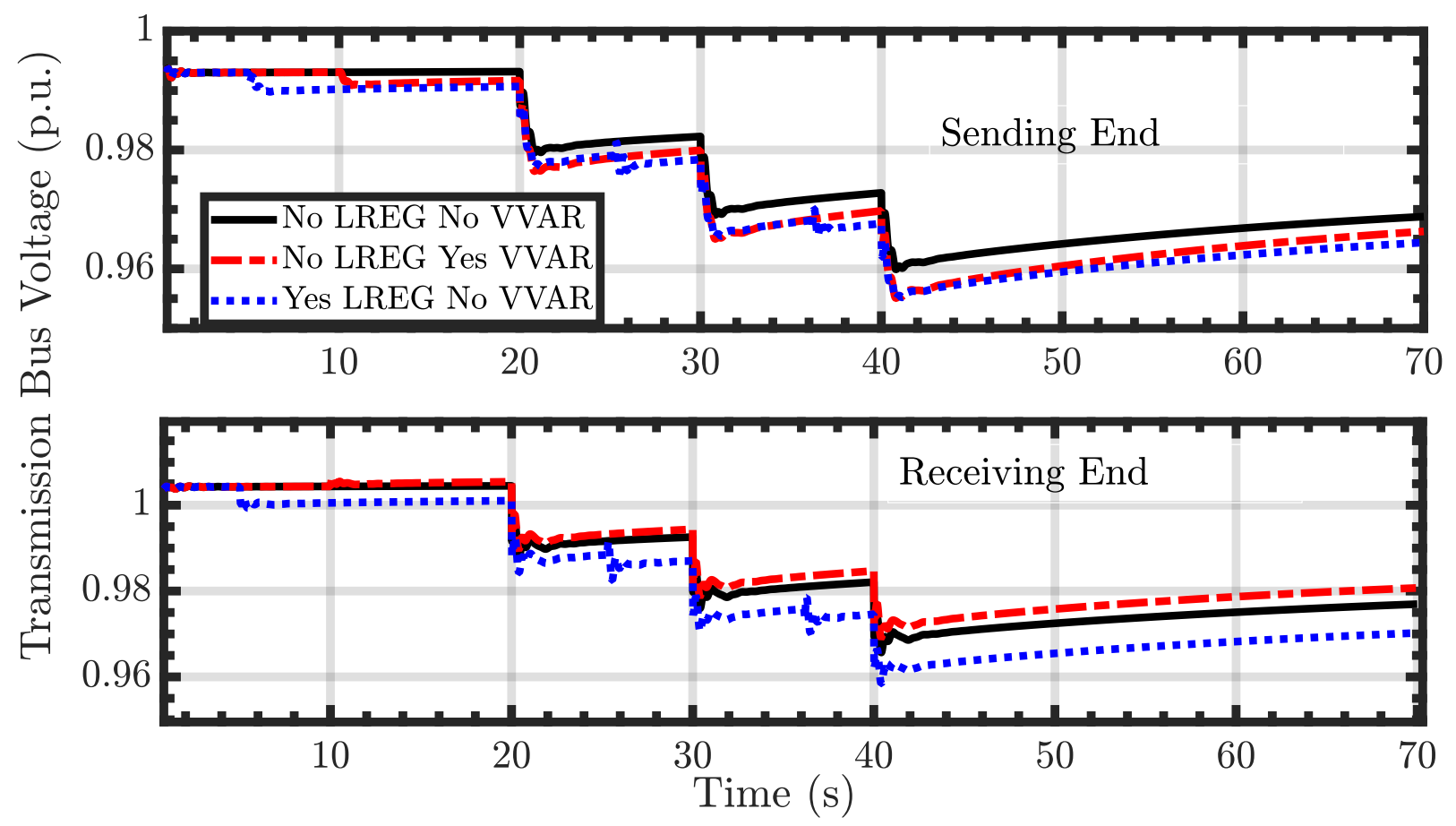

Figure 31 Transmission Bus Voltage Comparison (note: $L R E G=$ line regulation; VVAR $=$ VoltVAR ).

However, a different response is observed for the case that has enabled reactive power support from DERs. First, we note that the voltage at the receiving end of the tie-line is higher than the base case and the case with only voltage regulation from line regulators. This result occurs because DERs provide reactive power support by injecting the reactive current into the system, which is different from a line regulator's action that changes the voltage in the distribution system by changing the transformer tap's position. As the load increases in the system, the voltage drops as increased current is drawn; however, without the assistance of a line regulator to maintain the distribution system voltage, the voltage-dependent loads themselves start to draw lesser amounts of current. In addition, as the DERs notice the drop in voltage, they inject reactive current into the system, thereby offsetting the reactive current that would have been drawn from the BES by the loads. Because of this reactive power offset from the DERs, the receiving-end voltage will not fall as low as it does in the case where only the line regulators provide voltage support, and it is better than the base case where no form of support is provided. 
This result shows that the voltage support from DERs can support the BES voltage and possibly delay or avoid voltage instability in the system.

Figure 32 compares the active and reactive power flowing into the distribution system for the different cases. The reactive power consumed by the distribution system falls in the case where the DERs provide voltage support because of the reactive power support from the DERs. As the load on the receiving end of the transmission system increases, the voltage in the receiving end falls; and as the distribution system loads, in our studies, are voltage dependent, we see a fall in power consumption in the distribution system in the base case that receives no form of reactive power support or voltage regulation. With voltage regulation from the line regulator, the power drawn by the distribution system increases slightly, causing more current to be drawn from the transmission system and eventually leading to lower voltage levels in the transmission system (see Figure 31).

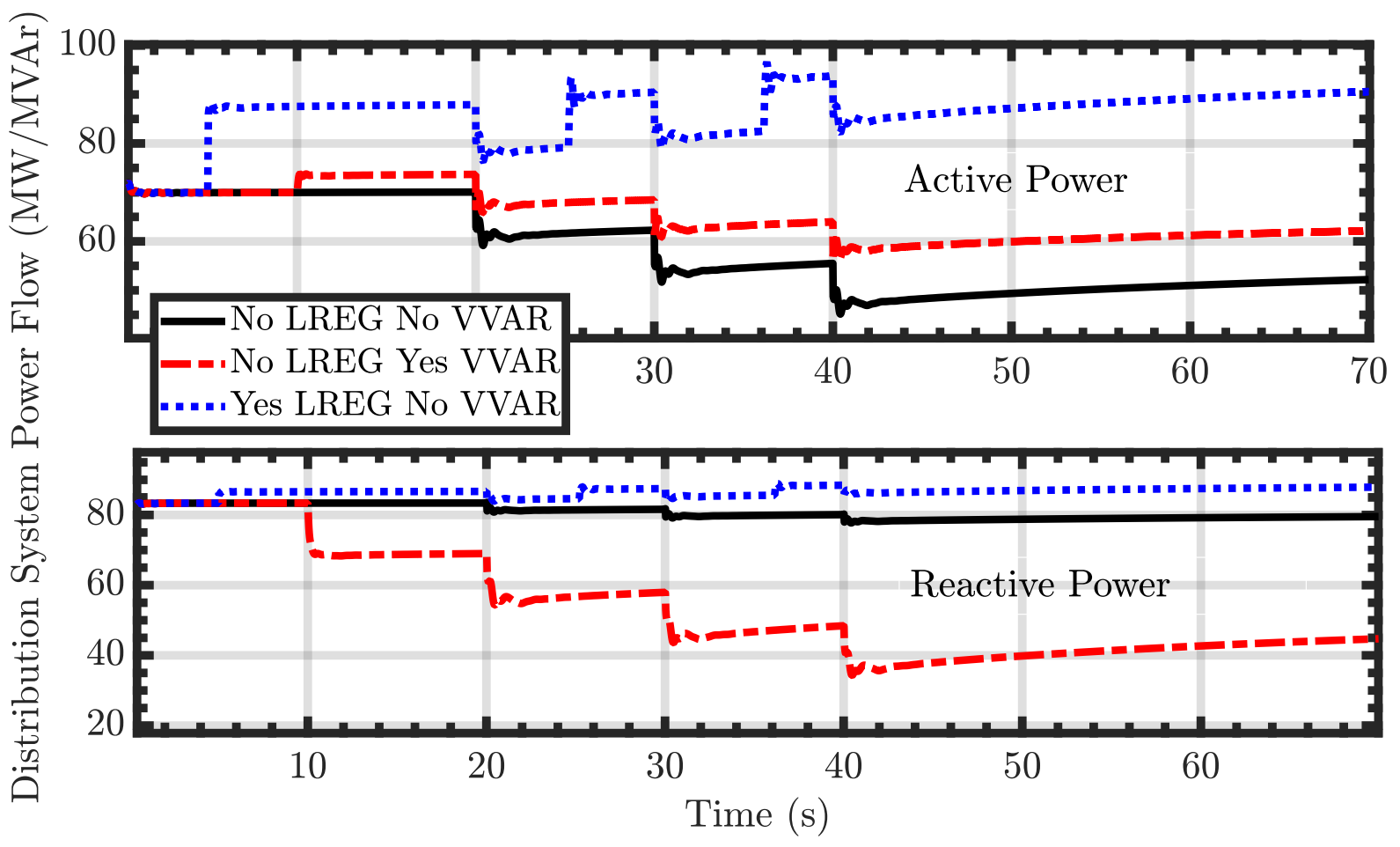

Figure 32 Distribution System Substation Power Flow Comparison.

Because of significant amounts of reactive power support from the DERs, along with lower voltage at the distribution system connected to the receiving end of the transmission bus, the reactive power flowing into the distribution system can be observed to be dropping, which eventually leads to a better voltage in the receiving end of the transmission bus as compared to the base case.

Figure 33 shows the tie-line active power flow between Areas 1 and 2 for the different cases considered. The lower value of the tie-line power flow for the case without voltage 
regulation from line regulators and reactive power support from the DERs is associated with the reduced active power consumed by the distribution system as shown in Figure 32. Figure 31 shows the comparison for the terminal voltages of the two tie-lines connecting Areas 1 and 2 for the different cases considered. As expected, the receiving-end voltage of the transmission bus is lower for the cases with line regulators enabled. With the line regulators enabled, the distribution system voltage increases, which leads to the result that the distribution system consumes more active power compared to the two other cases with line regulators off (see Figure 32). This increased power consumption leads to increased tie-line power flow, as well as reduced receiving-end voltage. Because of the reactive power support from DERs, the receiving-end voltage for Case 2 is higher than it is for Case 1, which leads to the voltage-dependent load drawing more power, thereby increasing the tie-line power flow.

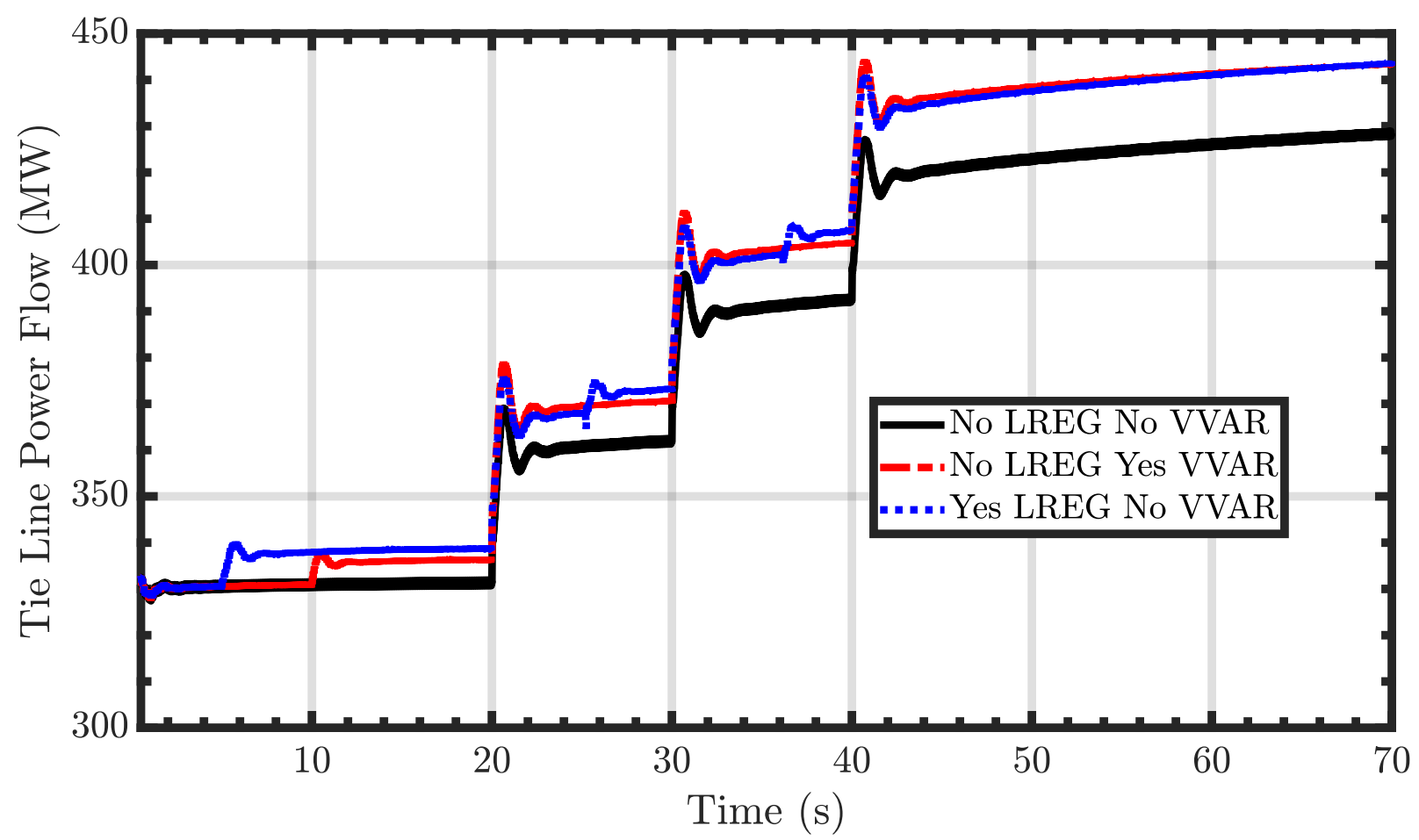

Figure 33 Tie-line Active Power Flow Comparison.

Figure 34 compares the reactive power output for the different cases considered. The reactive power injection from the DERs is only enabled for the case where Volt-VAR support is enabled. The figure shows that the reactive power support from the DERs increases as the distribution system voltage connected to the receiving end of the BES drops, which is caused by the increase in the load connected to the receiving end of the BES. Because of the reactive power injected from the DERs as shown in Figure 34, the reactive power demand in the distribution system is observed to fall in Figure 32. It is also of note that the reactive power support from DERs in Figure 34 differs from each source, which is dependent on the voltage at the PCC of the individual DER. 


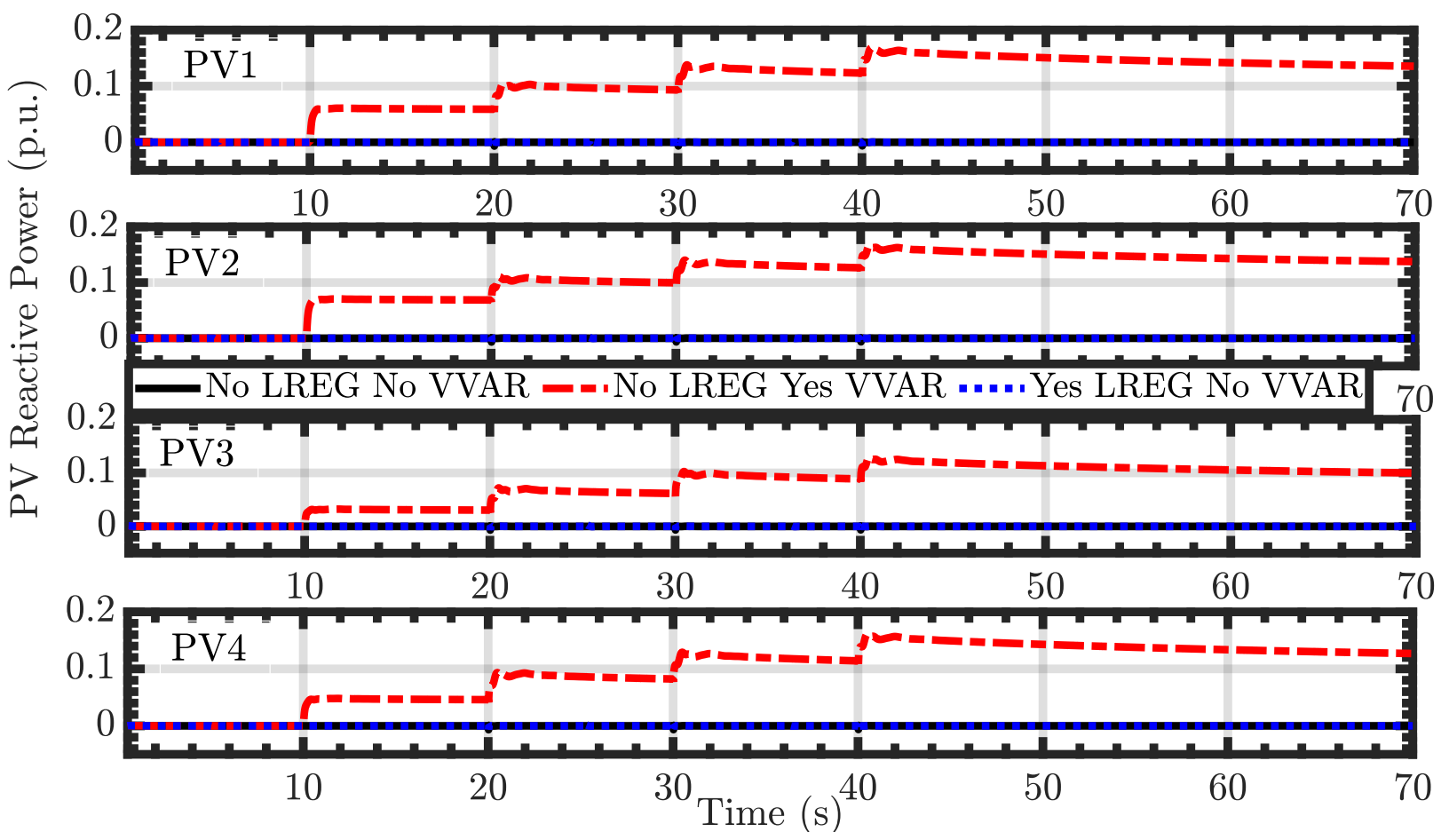

Figure 34 PV Reactive Power Output Comparison.

\subsubsection{Impact of Different DER Volt-VAR Curves (under Same Time Delay) on BES Voltage Regulation}

This section covers how BES operation is affected by DERs' various slopes of the VoltVAR curve with time-delayed voltage regulation. This study introduces a time delay of 10/60 seconds between the reference generated by the Volt-VAR curve and the reference provided to the inverter control. The time delay considered in this study represents the sum of the response delay and other inherent delays in the DER control loop. While keeping the VVC time delay constant, this case varies the slope of the Volt-VAR curve to evaluate the impact of different Volt-VAR settings implemented with the DERs on the voltage regulation of the BES and the distribution system.

The average dynamic model represents the DERs, and Figure 30 illustrates the Volt-VAR curve that has been implemented. The settings used for the Volt-VAR curve are as follows:

a. Setting 1 (mild Volt-VAR):

$\mathrm{V}_{\mathrm{L}}=0.50$ p.u., $\mathrm{V}_{1}=0.82$ p.u., $\mathrm{V}_{2}=1.00$ p.u., $\mathrm{V}_{3}=1.00$ p.u., $\mathrm{V}_{4}=1.18$ p.u., $\mathrm{V}_{\mathrm{H}}=1.2$ p.u.

b. Setting 2 (normal Volt-VAR):

$\mathrm{V}_{\mathrm{L}}=0.50$ p.u., $\mathrm{V}_{1}=0.90$ p.u., $\mathrm{V}_{2}=1.00$ p.u., $\mathrm{V}_{3}=1.00$ p.u., $\mathrm{V}_{4}=1.10$ p.u., $\mathrm{V}_{\mathrm{H}}=1.2$ p.u.

c. Setting 3 (steep Volt-VAR):

$\mathrm{V}_{\mathrm{L}}=0.50$ p.u., $\mathrm{V}_{1}=0.98$ p.u., $\mathrm{V}_{2}=1.00$ p.u., $\mathrm{V}_{3}=1.00$ p.u., $\mathrm{V}_{4}=1.02$ p.u., $\mathrm{V}_{\mathrm{H}}=1.2$ p.u. 
In the settings, $\mathrm{V}_{\mathrm{H}}$ and $\mathrm{V}_{\mathrm{L}}$ represent the upper and lower voltage limits for continuous operation of DERs. Beyond these voltage levels, the DERs' output saturates at the allowed maximum or minimum of the reactive power output capability. (The above settings are referred to as mild Volt-VAR, normal Volt-VAR, and steep Volt-VAR, respectively, in the balance of the document.) Setting 1 and Setting 3 are based on the maximum and minimum allowable settings for a Volt-VAR curve as suggested in IEEE Standard 1547-2018, and Setting 2 is the average of these two settings.

Please note that in all of these cases, there is no dead band in the Volt-VAR curve. The system operating condition in this case is tabulated in Table B-1.

In this section's case study, the test power system is operating at normal conditions throughout, and the Volt-VAR control from DERs in Area 2 is activated at 5 seconds. The purpose of this study is to evaluate the impacts of slower response times of DERs (due to delays in the control loop) in combination with different Volt-VAR settings on the overall stability of the system.

The literature [42] demonstrates that the delay in the response of the Volt-VAR-based voltage regulation from DERs can lead to system voltage oscillations and even instability; and thus the response times of Volt-VAR curves should be carefully evaluated. This study indicates that slow response coupled with steep Volt-VAR settings may cause system instability; thus, the setting of Volt-VAR curves should be well coordinated with the response lag of the DERs. It is also important to note that while the line regulators are modeled in the test case, their operation is disabled.

In this test case, neither the drop in the system frequency nor the voltage deviation is sufficient to trigger the frequency- and voltage-related protection settings of DERs. In addition, the Frequency-Watt control of the DERs is disabled in this case.

Figure 35 shows the PV reactive power output for the four PV systems implemented in the test distribution system for each of the three different Volt-VAR settings and with the time delay fixed at $0.1667 \mathrm{~s}$. The figure shows that before the Volt-VAR support is enabled, the DER can maintain its reactive power output close to 0 . Then, when the Volt-VAR control is enabled, the reactive power output from DERs settles down to a constant value depending on the voltage deviation from the nominal set-point for both the mild Volt-VAR setting and normal Volt-VAR settings. However, for the case with steep Volt-VAR, as soon as the Volt-VAR control is enabled, the reactive power output starts oscillating for all four PV systems. What we have observed in this scenario is that the local DERs' Volt-VAR controller becomes completely unstable when a steep Volt-VAR setting is coupled with slowly responding DERs. 


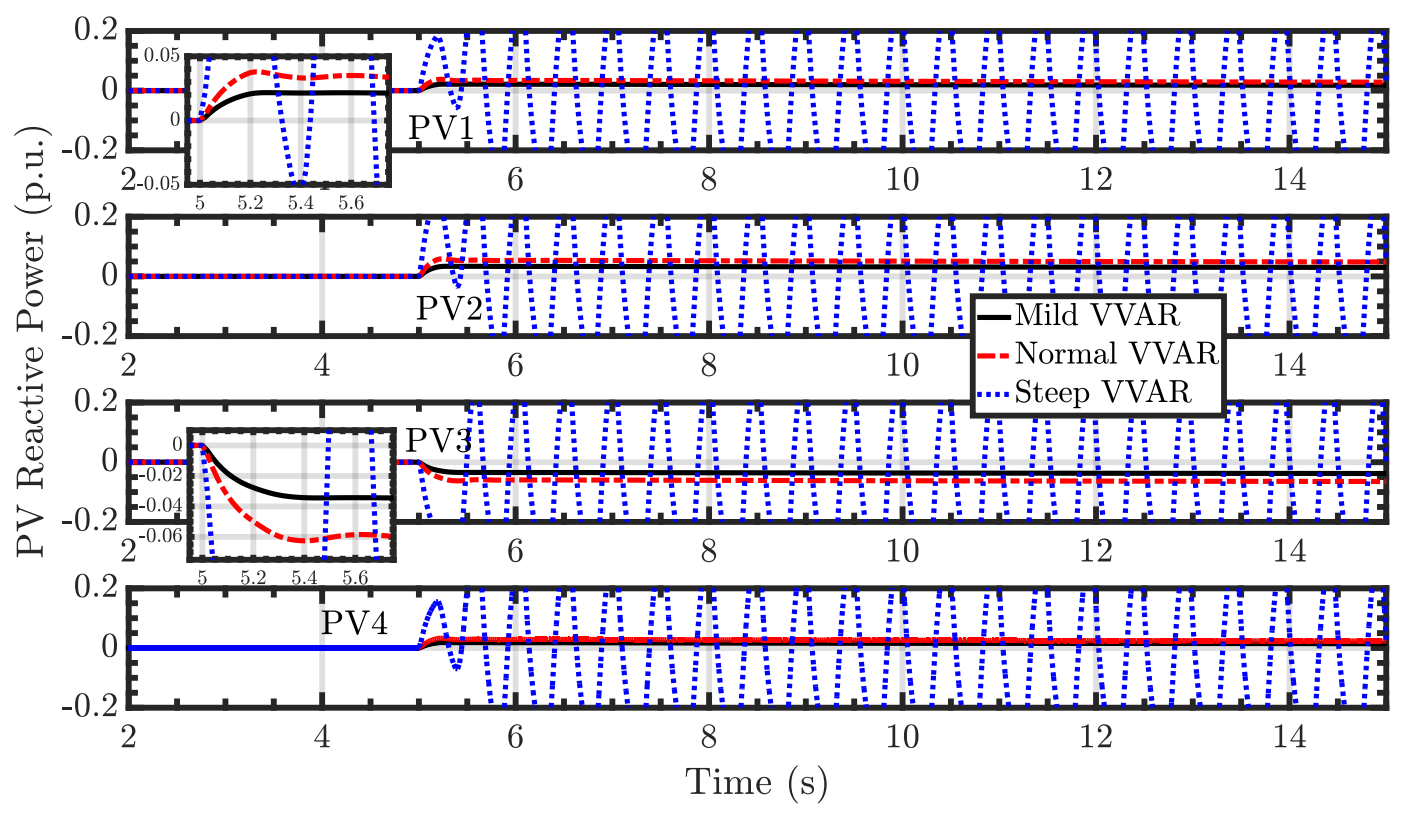

Figure 35 PV Reactive Power Output Comparison for Different Volt-VAR Settings.

The oscillations are caused as a small change in voltage demands a larger variation in reactive power output, which, when delayed, has a delayed effect on the voltage; thus, the voltage is never corrected at the proper time. This results in further voltage deviation, causing a feedback loop that leads to oscillatory behavior.

Figure 36 shows the PV PCC voltage comparison for the different cases considered. The figure shows that for the case with the mild and normal Volt-VAR settings, the PV PCC voltage settles down to a value close to the nominal value after some minor oscillations upon the enabling of the Volt-VAR control. However, with the steep Volt-VAR setting, the PV PCC voltage magnitude oscillates continuously and does not settle down. Beyond a certain slope threshold for volt-VAR settings, the case study [see Figure 35] indicates that small changes in voltages result in large changes in reactive power, causing even larger changes in the DERs' terminal voltage, as well as sustained oscillation of PV PCC voltage. This result suggests that the Volt-VAR settings for DERs should be coordinated with their response times, so as to avoid sustained voltage oscillations in the system. 


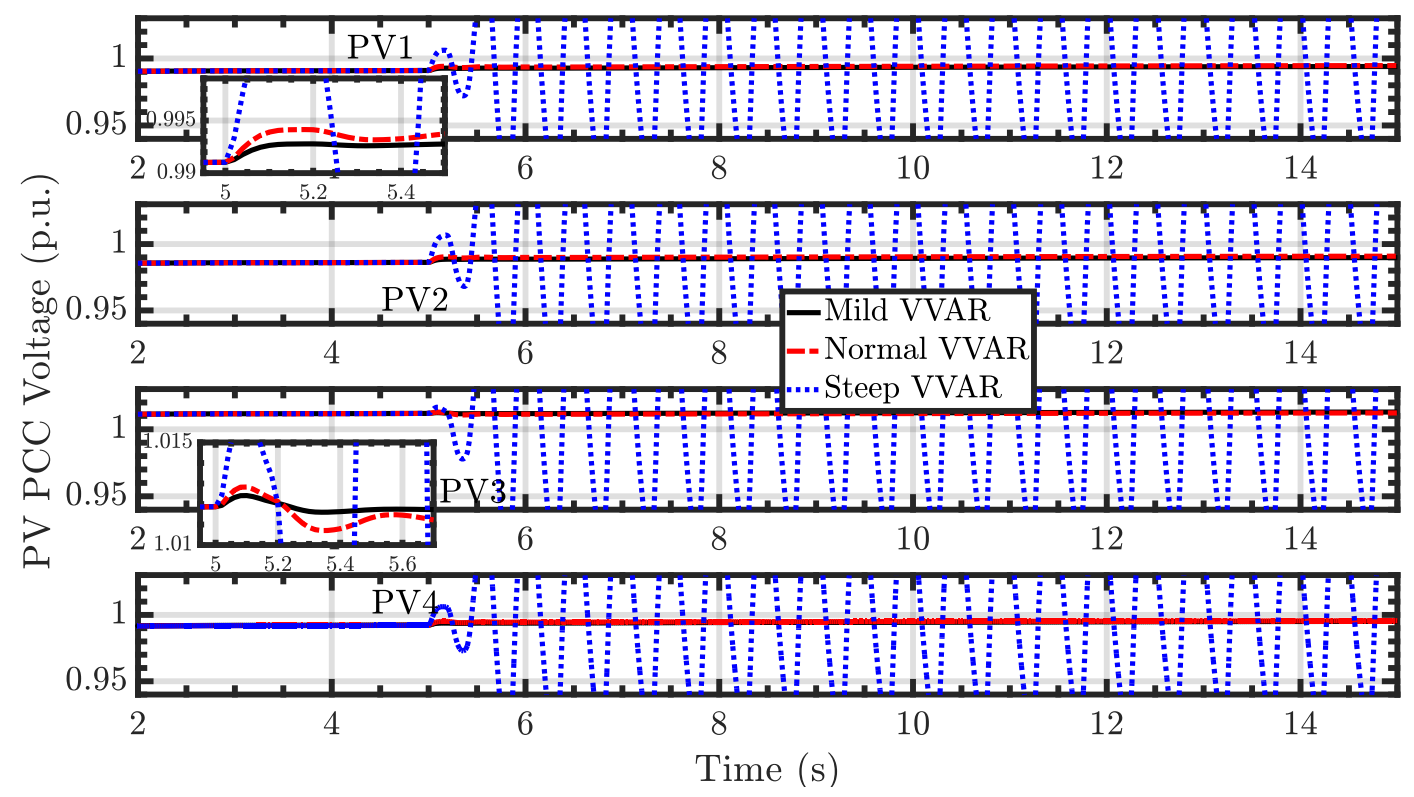

Figure 36 PV PCC Voltage Comparison for Different Volt-VAR Settings.

Figure 37 shows the comparison between the sending-end and receiving-end voltage of the tie-line connecting Area 1 to Area 2 of the test power system. The figure shows that when the steep Volt-VAR setting is applied, there are sustained oscillations on both sending- and receiving-end voltages and the oscillation magnitude is higher for the receiving-end voltage. This result shows that when the DER penetration level is high, the DER voltage regulation settings should be well coordinated, or the impact can be observed up to the transmission system.

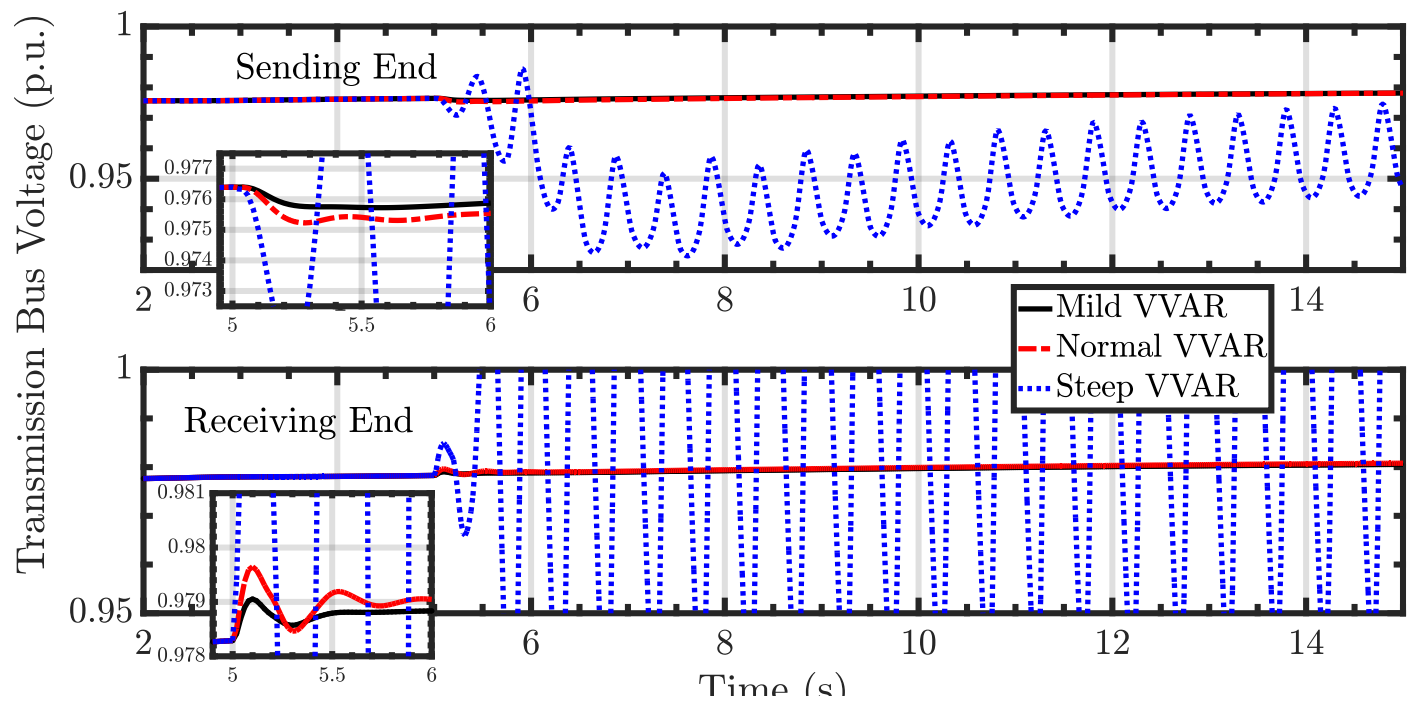

Figure 37 Tie-Line Bus Voltage Comparison for Different Volt-VAR Settings. 
Figure 38 and Figure 39 compare the active and reactive power, respectively, flowing toward the distribution system from the bulk power grid. The figures show that when the steep Volt-VAR setting is applied, the oscillations in the PV active and reactive power output can lead to oscillations in the active and reactive power flowing from the transmission system to the distribution system. When mild and normal Volt-VAR settings are applied, with reactive power support from DER, the reactive power consumed by the distribution system from the BES can be reduced; however, this event might lead to increased consumption of active power by the voltage-dependent loads in the distribution system.

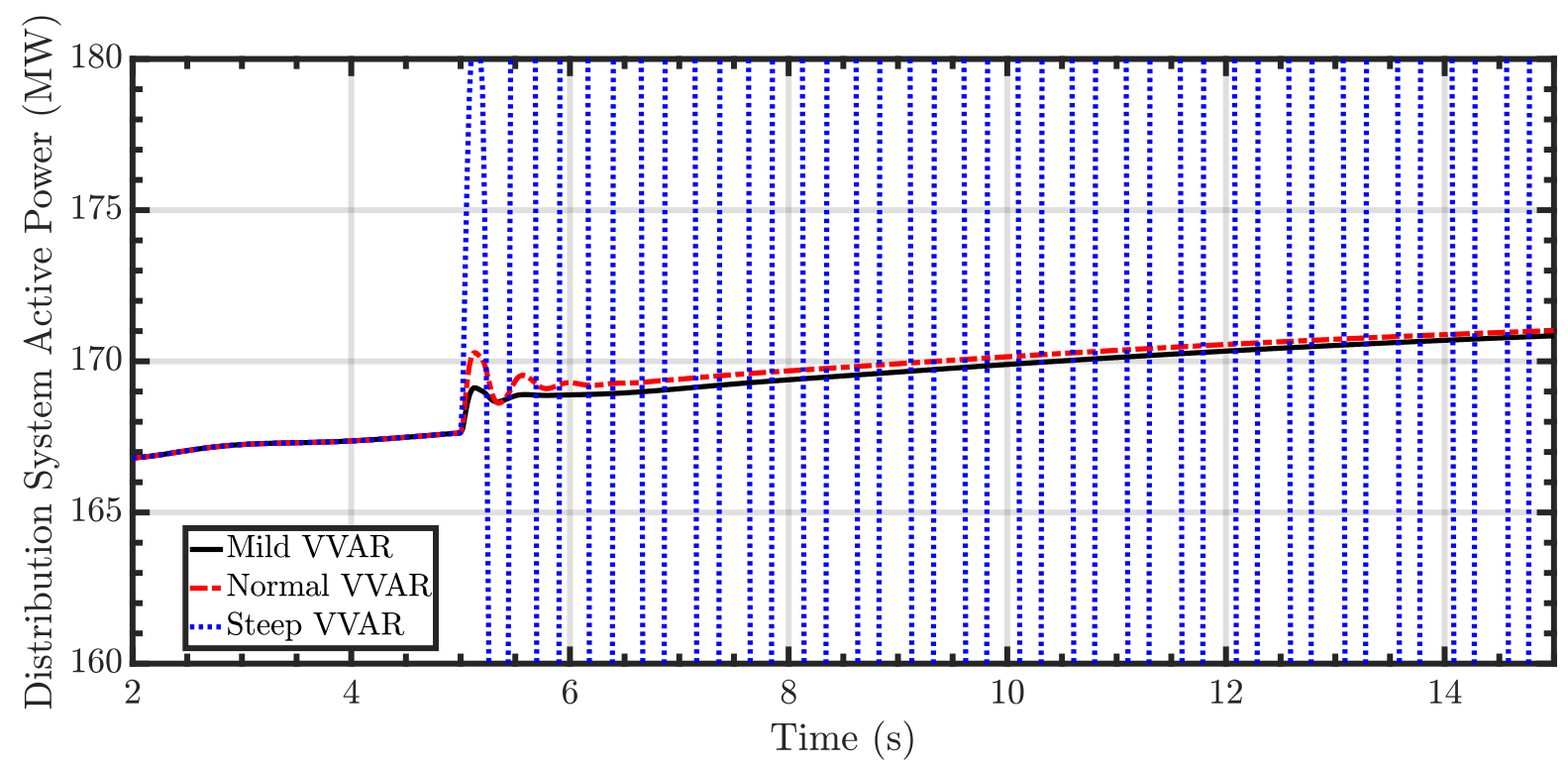

Figure 38 T\&D Boundary Bus Active Power Flow Comparison for Different Volt-VAR Settings.

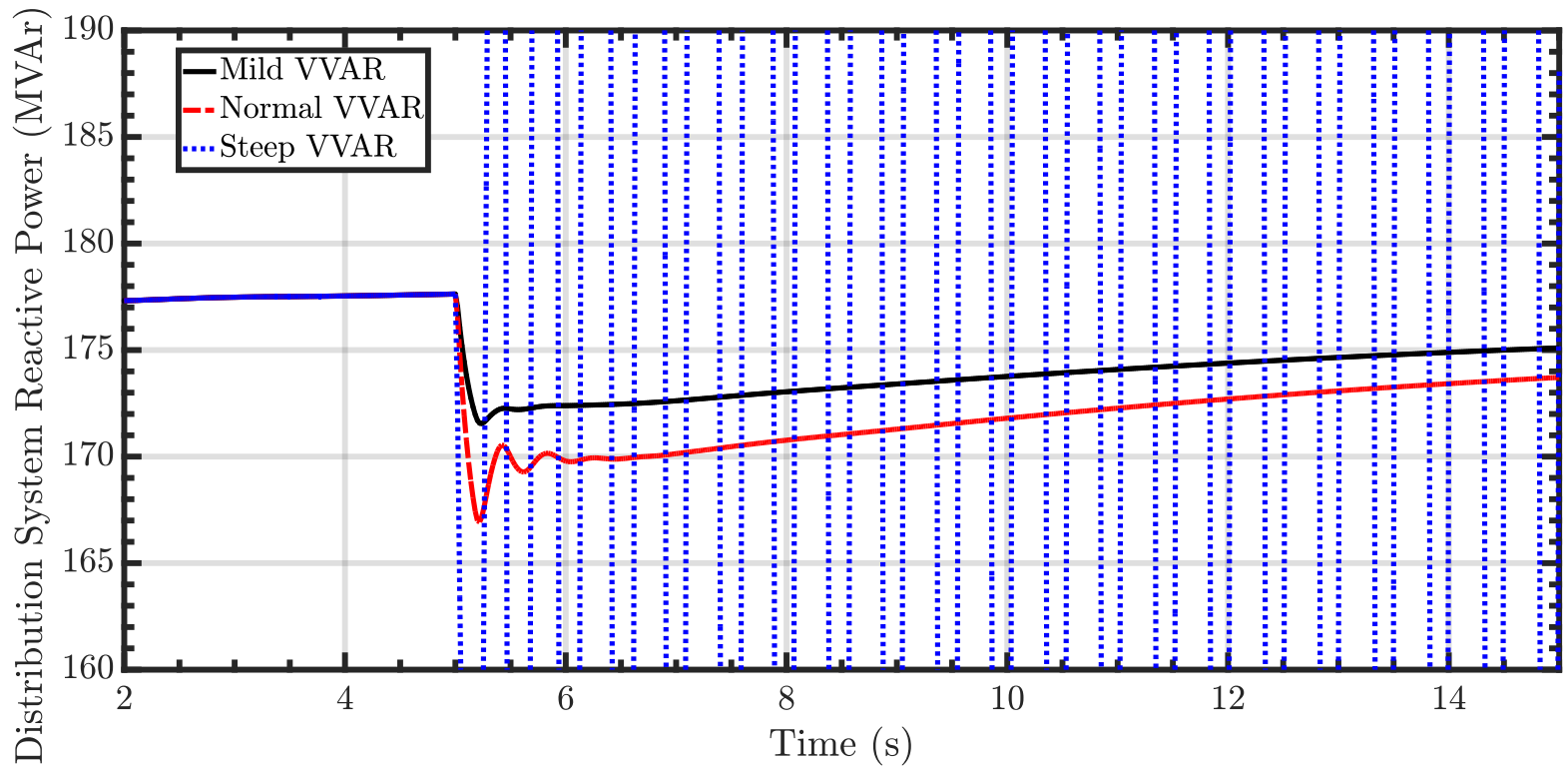

Figure 39 T\&D Boundary Bus Reactive Power Flow Comparison for Different Volt-VAR Settings. 
Figure 40 shows the comparison between the tie-line's active power flow with different Volt-VAR settings in DERs. The figure shows that in the case with mild and normal Volt-VAR curves, the oscillations in tie-line power flow are dampened. However, for the cases with steep Volt-VAR control, the oscillations in tie-line active power flow are sustained because of the sustained oscillations in the reactive power output of the DERs associated with the combination of time delay and steep Volt-VAR setting.

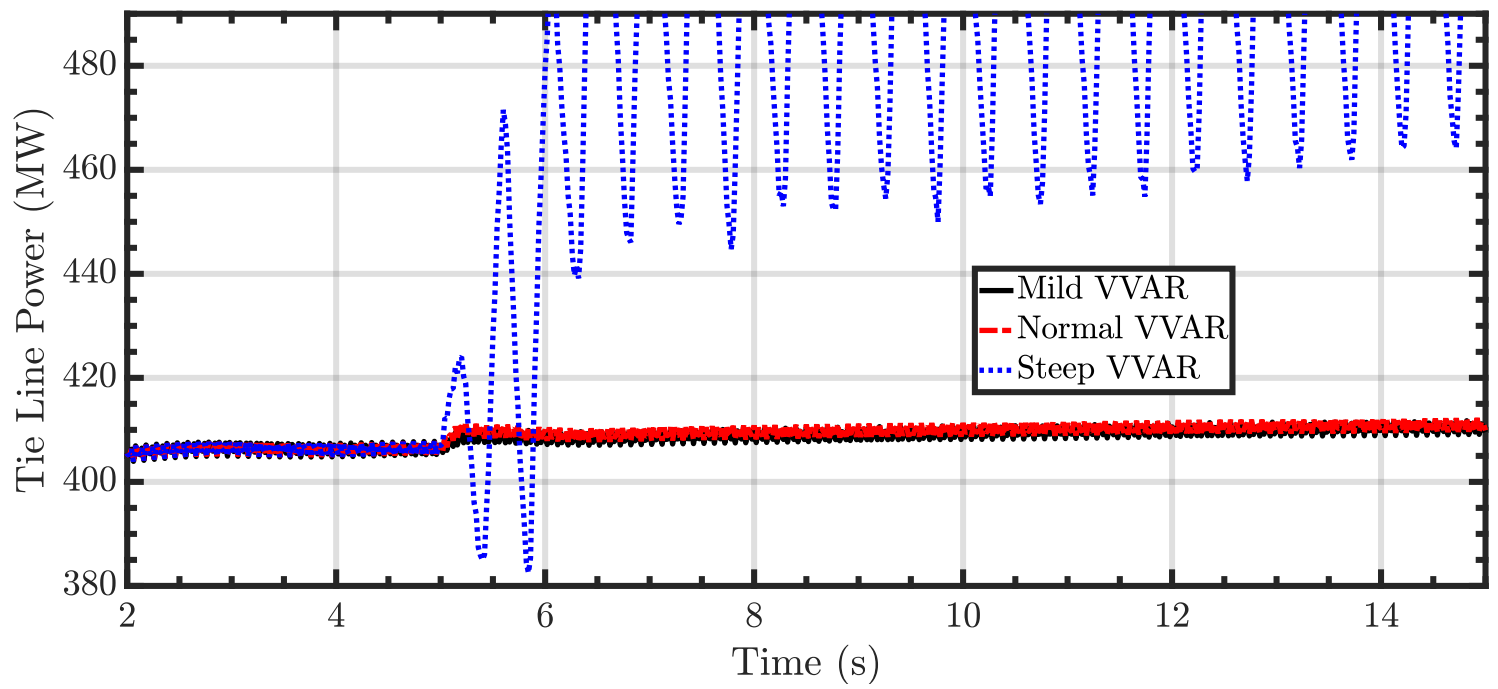

Figure 40 Tie-Line Power Flow Comparison for the Case with Different Volt-VAR Settings.

Figure 41 shows the generator rotor speed for different generators in the test system for the cases considered, where the effect of the steep Volt-VAR control is reflected on the rotor speed of nearby ${ }^{2}$ ? generators (G3 and G4 in Area 2). The figure shows that the rotor speed of generators G3 and G4, which are close to the modeled distribution system, have higher rotor speed oscillations as compared to generators G1 and G2. This result could also be attributable to the reduction in effective inertia in Area 2 that results from the larger penetration of DERs in Area 2. More studies are conducted to verify this finding.

\footnotetext{
${ }^{2}$ The nearby generators are the ones in the same area as the PV systems, in this case Area 2.
} 


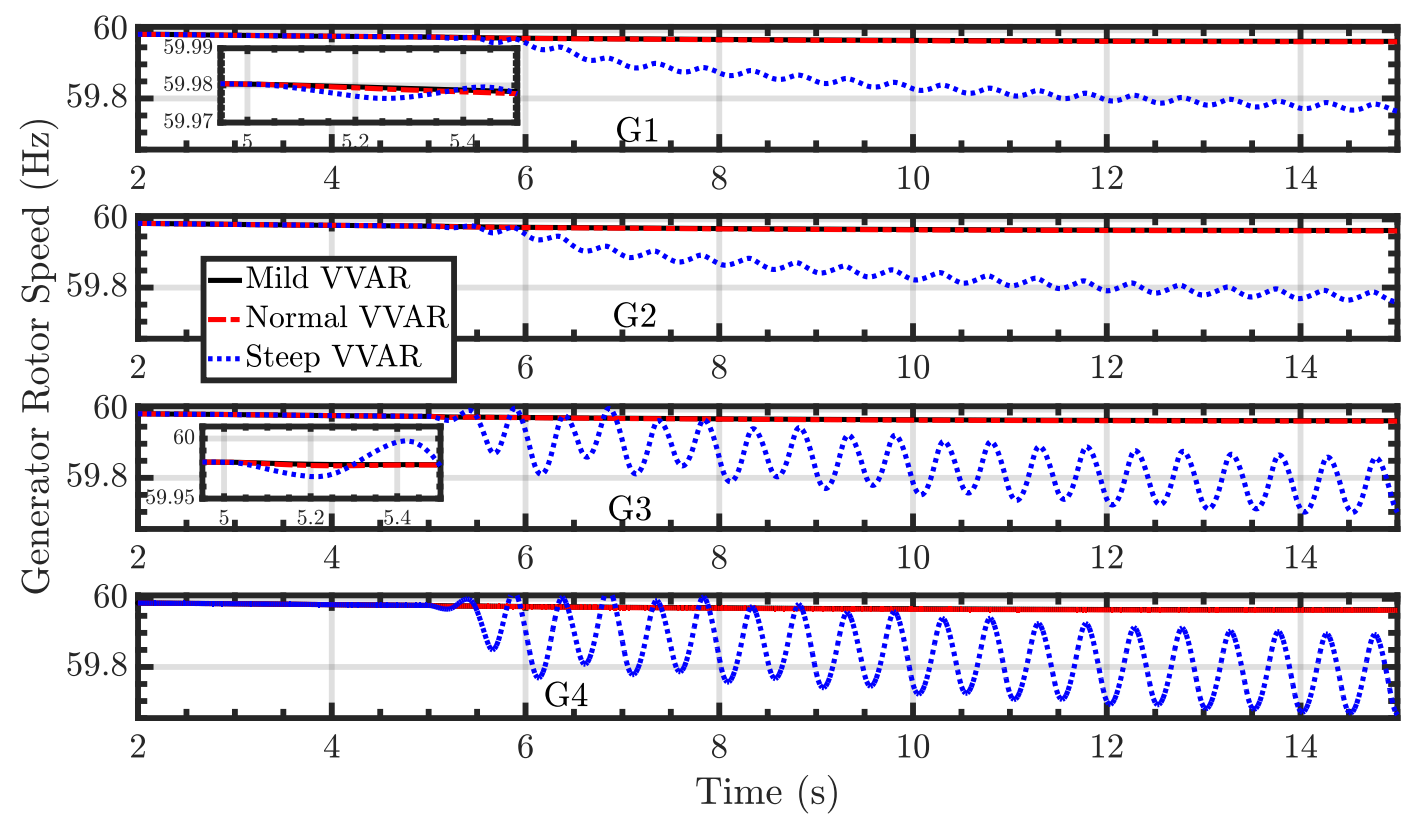

Figure 41 Generator Rotor Speed Comparison for Different Volt-VAR Settings.

\subsubsection{Impact of Different Time Delays of DERs' Volt-VAR Control on BES Voltage Regulation}

This section addresses the impact of different time delays in the DERs' Volt-VAR implementation on the voltage regulation of the distribution system and BES stability.

The setting used in the DER Volt-VAR curve for this study (based on [43]) is as follows:

$$
\mathrm{V}_{\mathrm{L}}=0.50 \text { p.u., } \mathrm{V}_{1}=0.99 \text { p.u., } \mathrm{V}_{2}=1.00 \text { p.u., } \mathrm{V}_{3}=1.00 \text { p.u., } \mathrm{V}_{4}=1.01 \text { p.u., } \mathrm{V}_{\mathrm{H}}=1.2 \text { p.u. }
$$

Please note that this particular test case has steeper settings as compared to other cases studied in this chapter.

In this section, the test power system is operating at normal conditions throughout the entire length of simulation, and the Volt-VAR support from DERs is activated at 10 seconds.

The scenarios considered are: (1) without any delay in the voltage regulation, and (2) with some delay in the voltage regulation. Specifically, the time delays considered in the response between voltage measurement and reactive power dispatch from the Volt-VAR controller are $\tau_{d}=0.0833 \mathrm{~s}$ and $0.16667 \mathrm{~s}$, respectively. For all of the scenarios considered in this case study, the response delay resulting from the filter time constant in the voltage measurement blocks is used. This response delay is different from the external delay, which is used to simulate the communication/signal transfer delay in the volt-VAR control loop. 
Figure 42 shows the PV reactive power output for the four PV systems both without delay and with different time delays in the Volt-VAR control. In the "without delay" case, there are no external delays placed in the loop. However, the response delay due to the filter time constant used in the voltage measurement blocks is still used. The figure shows that before the Volt-VAR control is enabled, DERs can maintain their reactive power output close to 0 . In addition, once the Volt-VAR control is enabled, for the scenario without any time delay, the reactive power output of the DERs is constant depending on the voltage deviation from the nominal set-point. However, for the scenarios with time delays, as soon as the Volt-VAR control is enabled, the reactive power output starts oscillating, and the magnitude and frequency of the oscillation are observed to be dependent on the amount of the time delay. What we have observed in this scenario is that the controller becomes completely unstable when DERs start operating in Volt-VAR control mode.

Oscillations are caused as small changes in voltage demand a larger variation in reactive power output for steep volt-VAR settings. On top of that, when the reactive power injection to compensate the voltage changes is delayed, the voltage change will not be effectively negated by the reactive power injection. Instead, this delayed reactive power injection can change the voltage further, causing a feedback loop that leads to oscillatory behavior as the voltage error cannot be driven to zero.
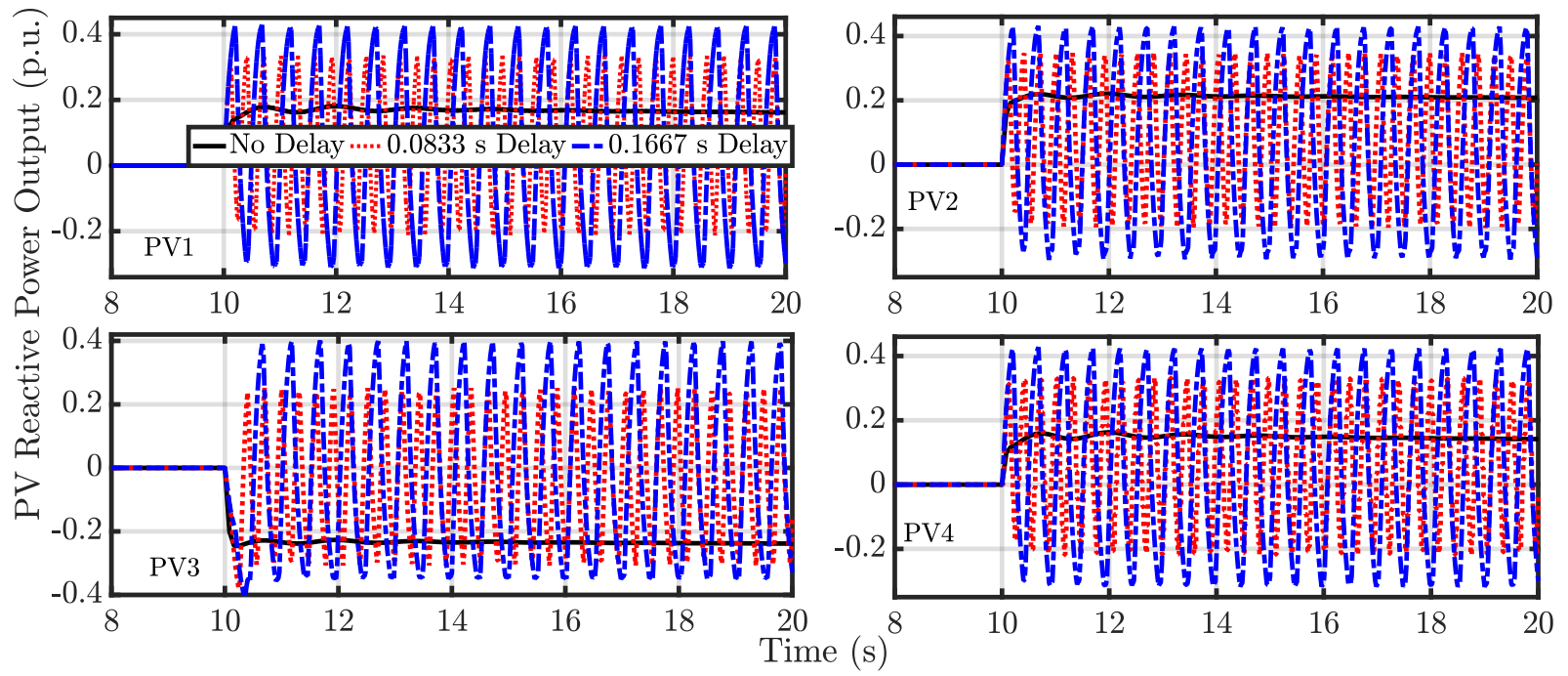

Figure 42 PV Reactive Power Output Comparison for the Scenarios with and without Time Delays in Volt-VAR Control.

Figure 43 shows the PV PCC voltage comparison for the different scenarios. The figure shows that for the scenario without any time delay in the Volt-VAR control, the PV PCC voltage settles down to a value close to the nominal value. However, with time delays in the Volt-VAR control, the PV PCC voltage magnitude oscillates. 

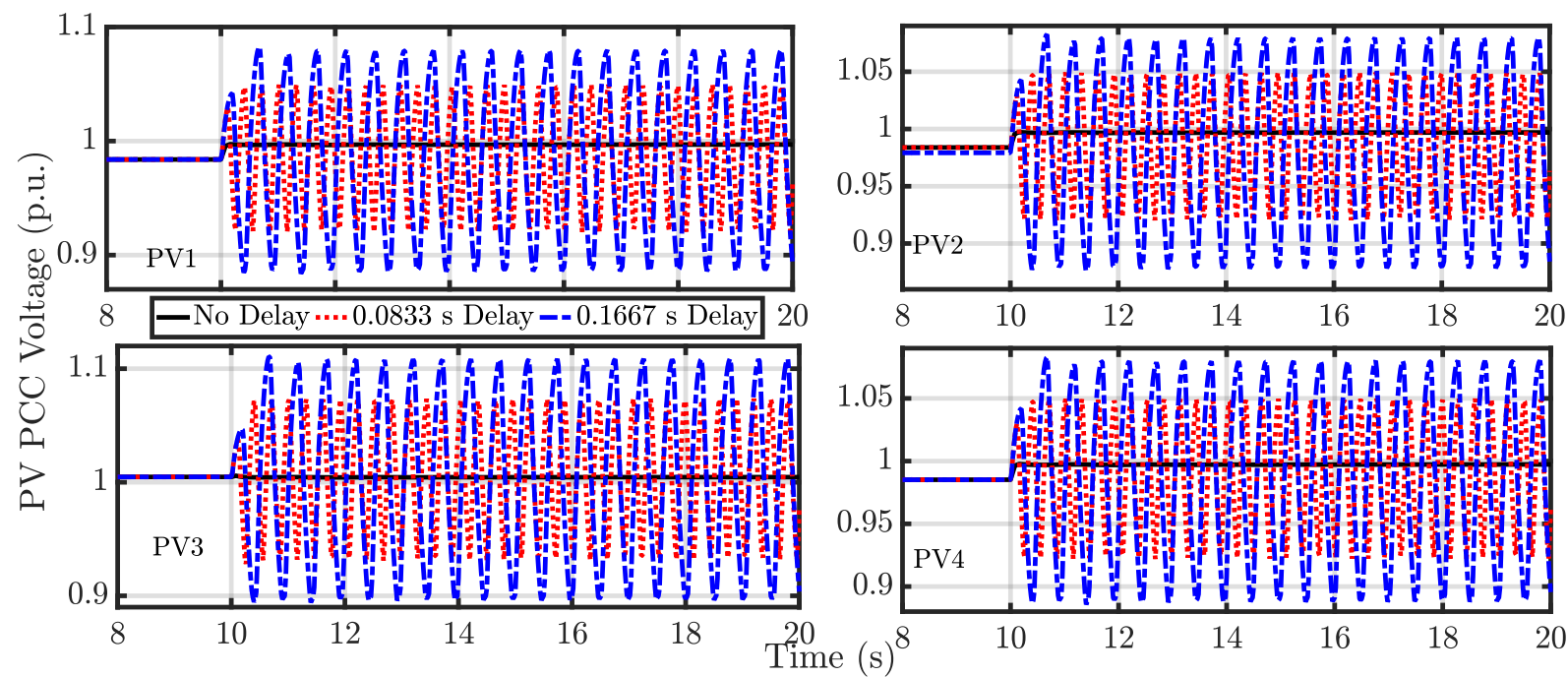

Figure 43 PV PCC Voltage Comparison for the Scenarios with and without Time Delays in Volt-VAR Control.

Figure 44 shows the comparison between the sending-end and receiving-end voltage of the tie-line connecting Area 1 to Area 2 in the test power system. The figure shows that with time delays, sustained oscillations are observed in both sending- and receiving-end voltages, and the oscillation magnitude is higher for the receiving-end voltage. In addition, a longer time delay contributes to a higher oscillation magnitude.

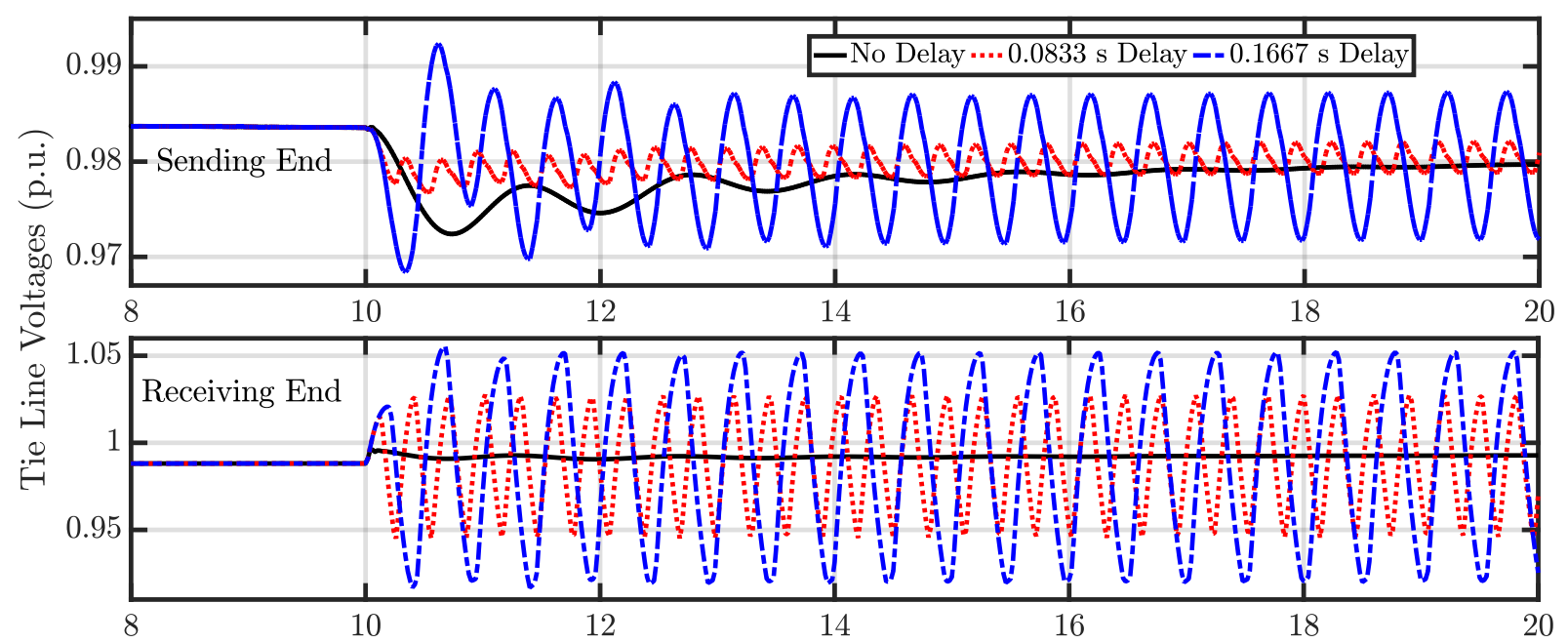

Figure 44 Tie-Line Bus Voltage Comparison for the Scenarios with and without Time Delays in Volt-VAR Control. 
Figure 45 compares the active and reactive power flowing toward the distribution system from the bulk power grid for different scenarios with and without time delays in Volt-VAR control. As the figure shows, when time delays are applied, and in combination with the very steep Volt-VAR curve, the oscillations in the PVs' active and reactive power outputs can lead to oscillations in active and reactive power flowing from the transmission system to the distribution system.

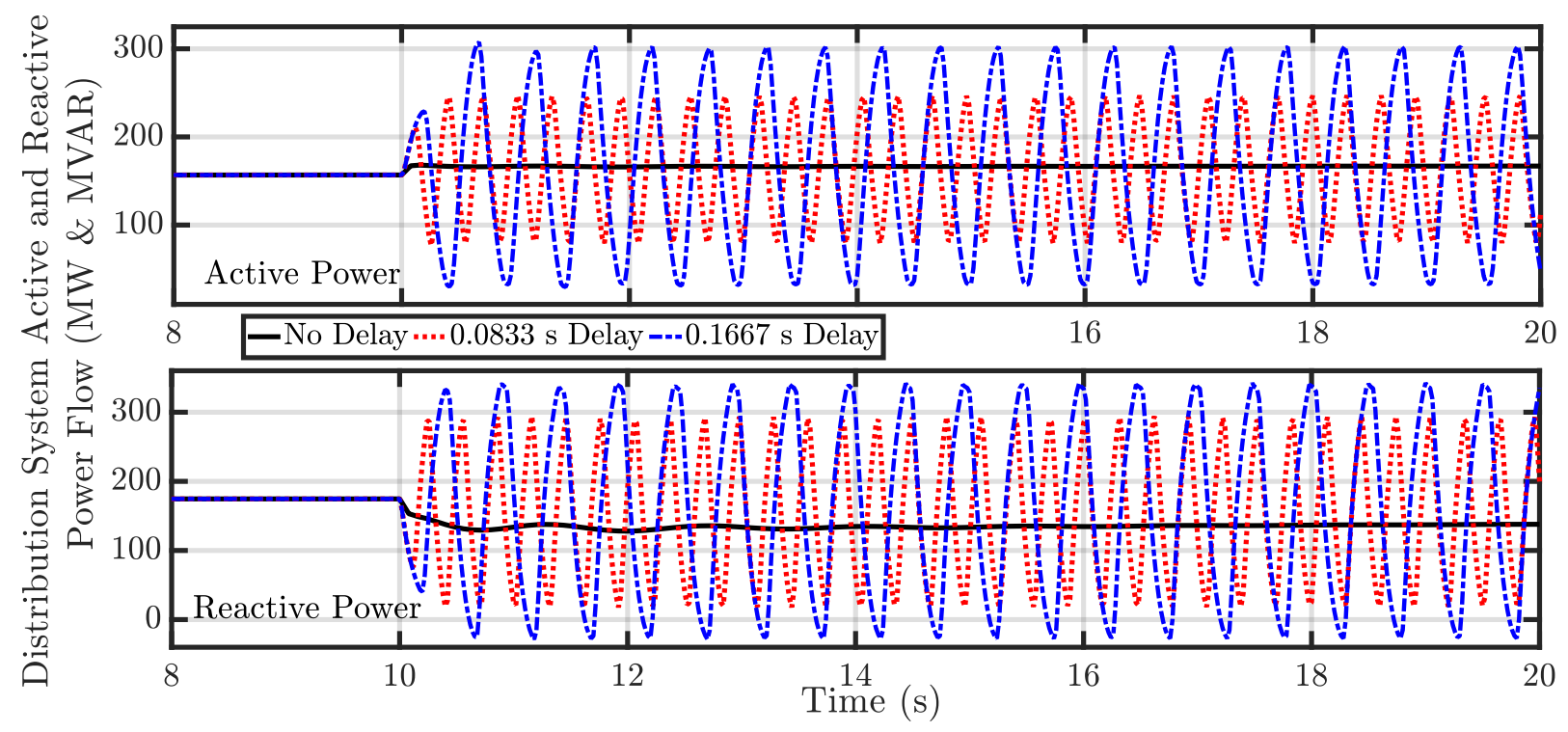

Figure 45 T\&D Boundary Bus Power Flow Comparison for the Scenarios with and without Time Delays in Volt-VAR Control.

Figure 46 shows the comparison of tie-line active power flows both with and without the time delays in the DERs VVC. The figure shows that for the scenario with no delays in the VoltVAR curve implementation, the oscillations in tie-line power flow are dampened. However, for the scenarios with delays in Volt-VAR control, the oscillations in tie-line active power flow are sustained due to the sustained oscillations in the reactive power output of the DERs associated with the combination of a time delay and a steep Volt-VAR setting. 


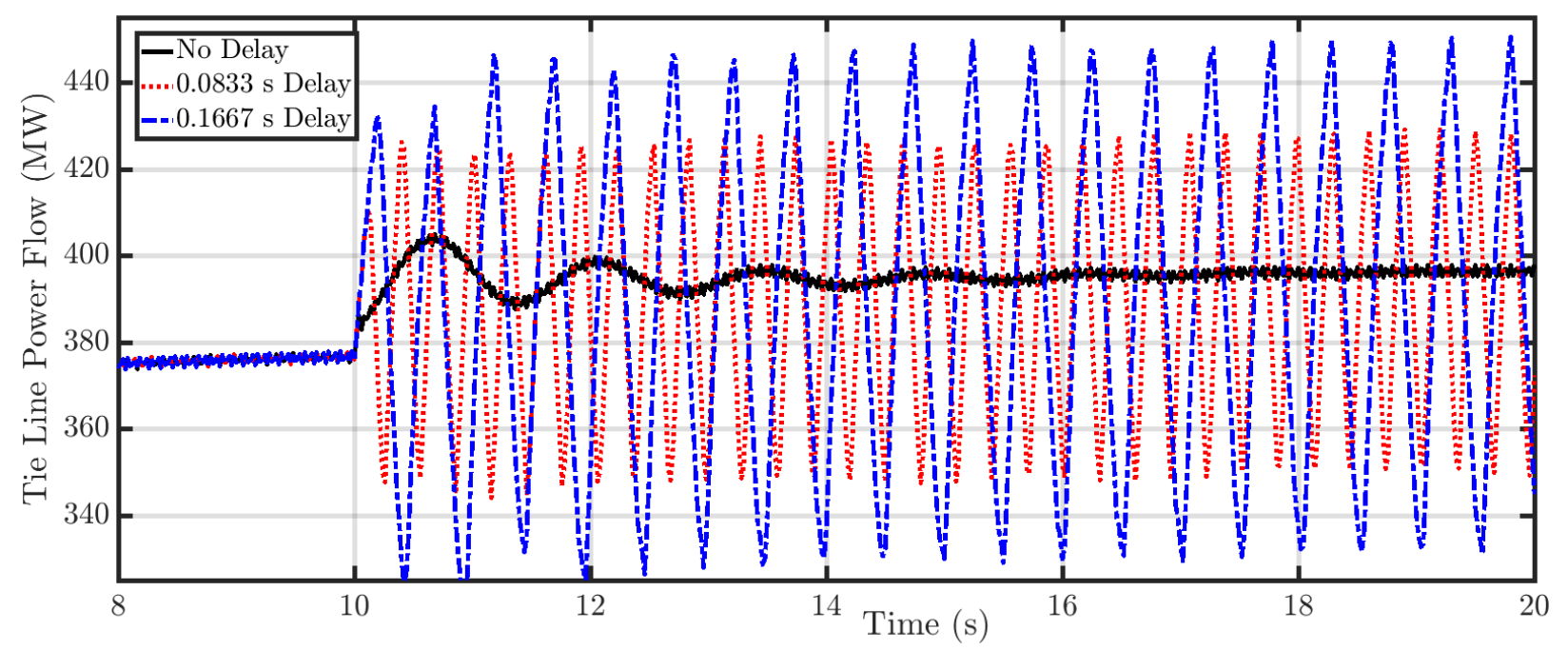

Figure 46 Tie-Line Power Flow Comparison for the Scenarios with and without Time Delays in Volt-VAR Control.

Figure 47 shows the generator rotor speed for different generators in the test system for the scenarios being considered. As the figure shows, the effects of time delays on the DERs' Volt-VAR control are reflected on the rotor speed of nearby generators. In particular, generators G3 and G4, which are close to the modeled distribution system have higher rotor speed oscillations as compared to generators G1 and G2.
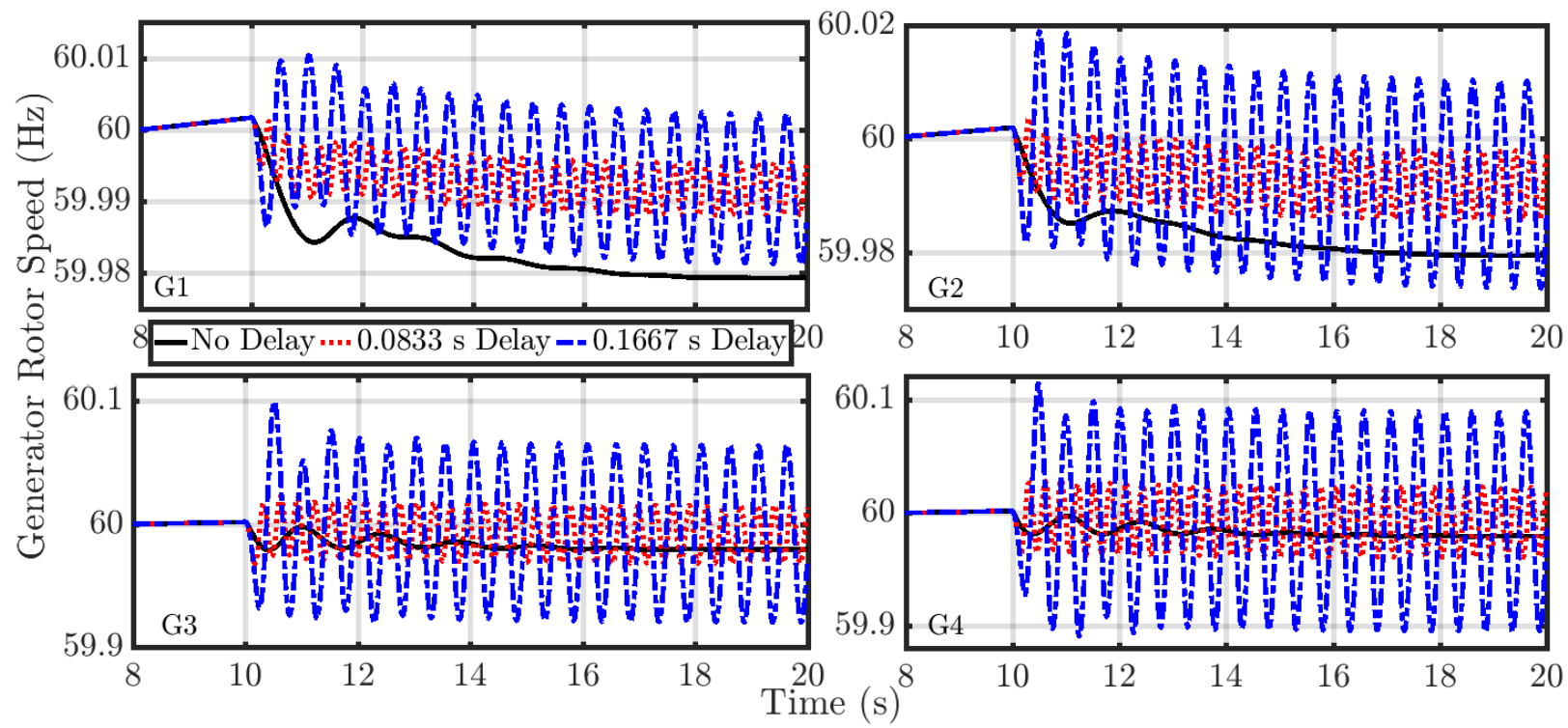

Figure 47 Generator Rotor Speed Comparison for the Scenarios with and without Time Delays in Volt-VAR Control. 


\subsubsection{Impact of Interaction between Multiple DERs with VVC Enabled on BES}

This case examines how the interactions between multiple DERs with different timedelayed voltage regulations can affect BES operation. The test distribution system has four DERs placed at different locations within the distribution system as shown in Figure 2. In this test, to study the effects of interactions between multiple DERs with the same Volt-VAR settings but with different time delays, the following cases were developed.

i. All four DERs with steep Volt-VAR curve and delay of 10/60 secs.

ii. Two DERs that are electrically most distant from one another with steep Volt-VAR curves and delays of 10/60 seconds and others with a steep volt-VAR curve and without a time delay.

iii. One DER with a steep Volt-VAR curve and delays of 10/60 seconds and others with a steep volt-VAR curve without delay.

The average dynamic model represents the DERs. The settings used for the Volt-VAR curves are as follows:

$$
\begin{aligned}
& \text { a. Volt-VAR Settings (Steep Volt-VAR): } \\
& \mathrm{V}_{\mathrm{L}}=0.50 \text { p.u., } \mathrm{V}_{1}=0.98 \text { p.u., } \mathrm{V}_{2}=1.00 \text { p.u., } \mathrm{V}_{3}=1.00 \text { p.u., } \mathrm{V}_{4}=1.02 \text { p.u., } \mathrm{V}_{\mathrm{H}}=1.2 \text { p.u. }
\end{aligned}
$$

The information about system operating conditions for this case is shown in Table B-1. In this case study, the test power system is operating at normal conditions throughout, and the VoltVAR control from DERs in Area 2 is activated at 5 seconds. Also note that, even though the line regulators are modeled in this case study, their controls are disabled for this case study.

The purpose of this study is to demonstrate that multiple DERs with voltage delay and steep Volt-VAR settings can interact with one another and can cause sustained system oscillations. It has been demonstrated that the sustained oscillations in the system occur when multiple DERs have steep Volt-VAR settings and a significant time delay; that is, the oscillations are not observed when only one DER had a time delay and steep volt-VAR settings and others do not. The oscillations are observed to die down when the electrically distant DERs had steep settings with significant delay. In this case study, neither the drop in the system frequency nor the voltage deviation is sufficient to trigger the frequency- and voltage-related protection settings of the DERs. In addition, the Frequency-Watt control of the DERs is disabled in this case.

Figure 48 shows the PV reactive power output for the four PV systems implemented in the test distribution system for the following case: with a fixed time delay of 0.1667 seconds, steep Volt-VAR settings, and with three different combination of DERs as mentioned in the introduction to Section 4.1.4. In all of the scenarios considered, all the DERs in the test distribution system will have exactly the same volt-VAR settings. What differs between the scenarios is the number of DERs with a communication delay. We observe that before the VoltVAR support is enabled, the DER can maintain its reactive power output at close to 0 . Furthermore, when the Volt-VAR control is enabled at time $t=5$ seconds, for the cases with multiple DERs with delay, the reactive power output from each DER oscillates and continues to oscillate or dampen depending on the number of DERs with time delay. 
This study suggests that for a distribution system with a high number of DERs, it is crucial that the DER voltage support settings be well coordinated. The results demonstrate that if the DER Volt-VAR settings are not well coordinated with their voltage settings, sustained voltage oscillation can occur within the distribution system, the impacts of which can be observed up to the BES level.

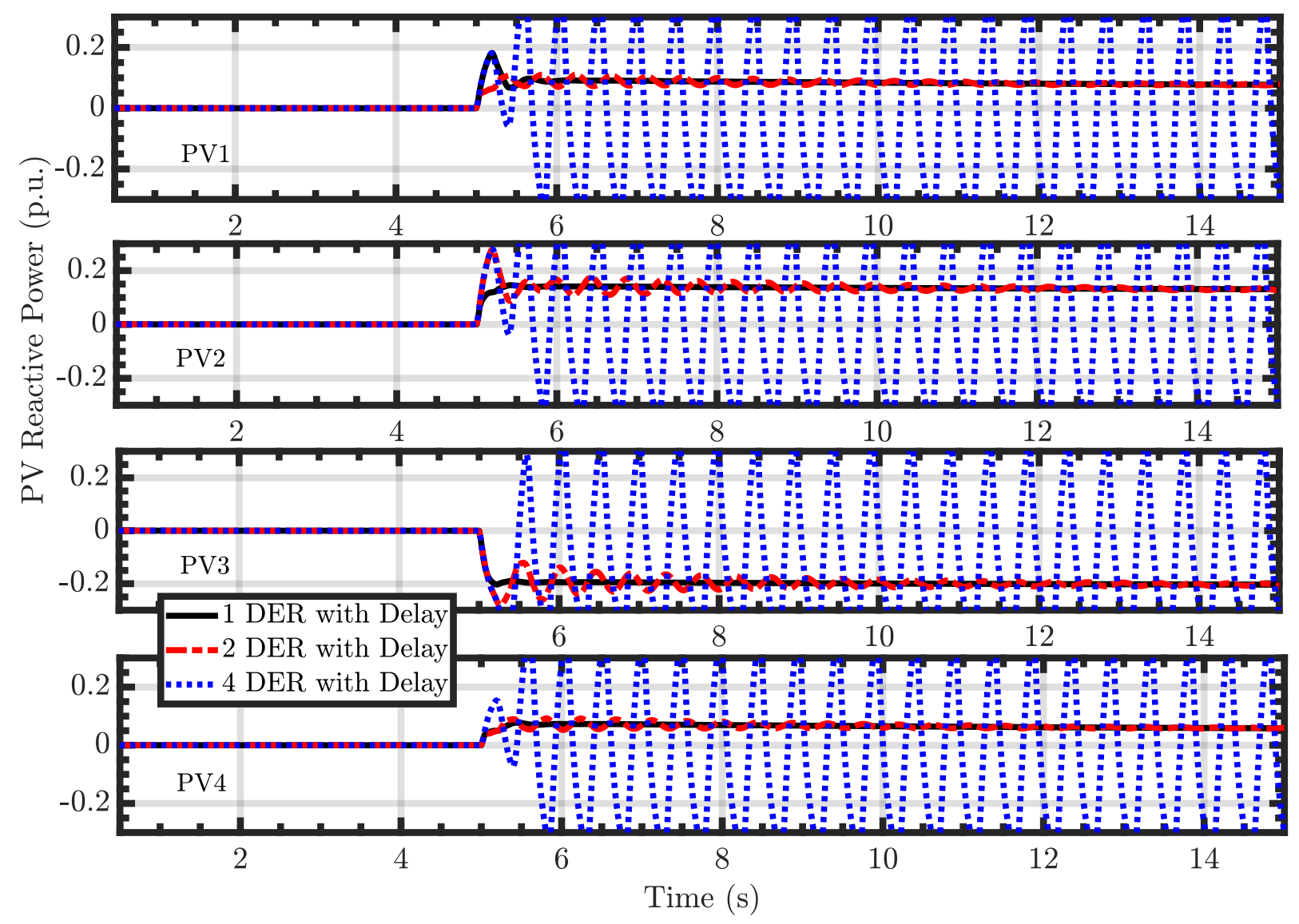

Figure 48 PV Reactive Power Output Comparison.

Figure 49 shows the PV PCC voltage comparison for the different cases, where for the case with time delay in one DER and two electrically distant DERs, the voltage oscillations dampen, and the voltage settles down close to the nominal value. However, with the same steep Volt-VAR settings but with a time delay in all four DERs, the PV PCC voltage magnitude oscillates and does not settle. Figure 49 also shows that as the number of DERs with time delay increases, the oscillation magnitude in the PV PCC voltage increases and can finally become unstable beyond a certain threshold. This result suggests that the oscillations were caused by the interaction among the DER Volt-VAR settings and should be coordinated with their response times to avoid such interactions. 


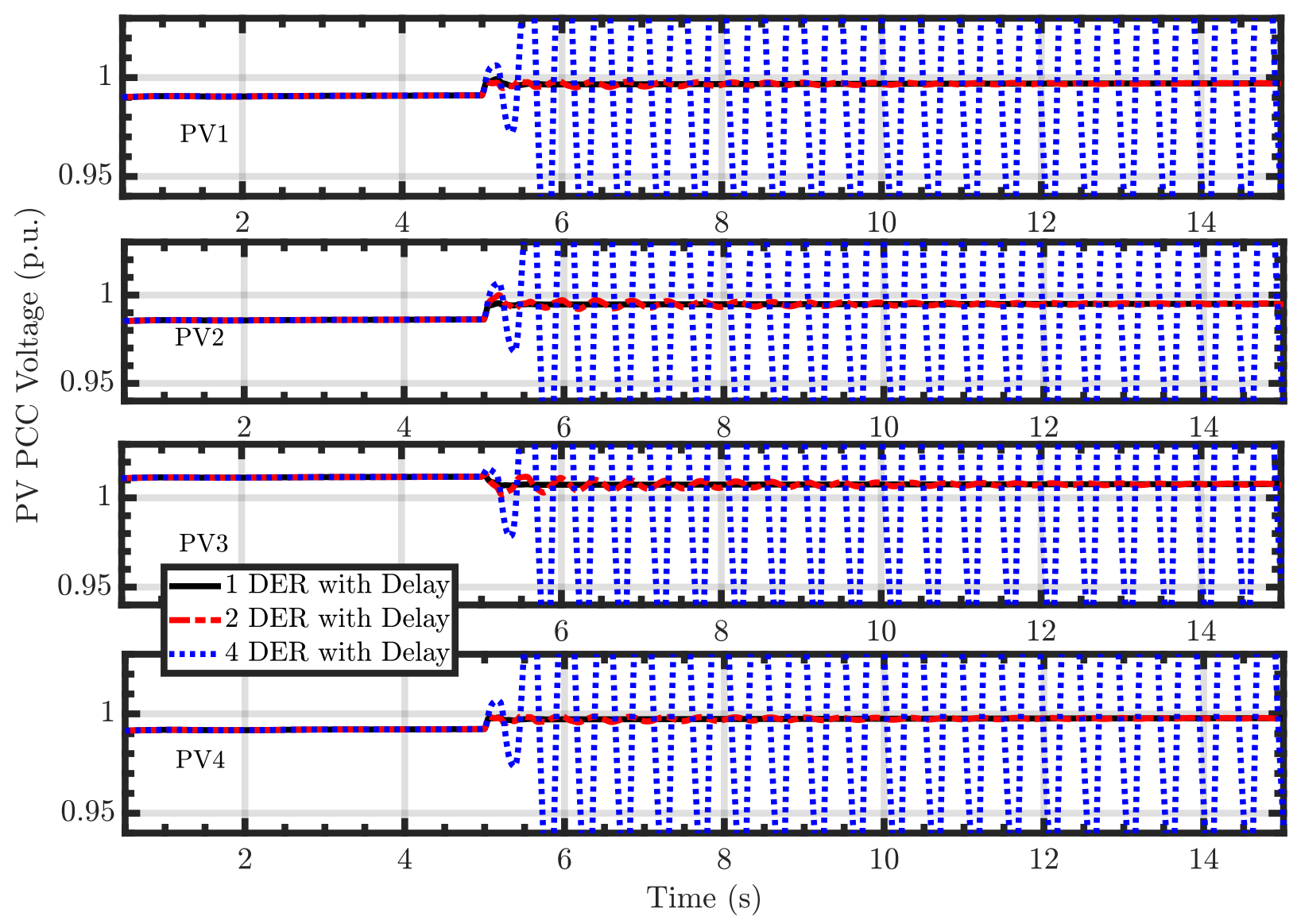

Figure 49 PV PCC Voltage Comparison.

Figure 50 shows the comparison between the sending-end and receiving-end voltage of the tie-line connecting Area 1 to Area 2 in the test power system. As the figure shows, with the oscillatory reactive power injection from DERs in Area 2 because of the interaction between multiple DERs with steep Volt-VAR settings and time delay, the receiving-end voltage has more variations as compared to that of the sending end. This result shows that when the DER penetration level is high, the DER voltage regulation settings should be well coordinated, or the impact may be observed up to the transmission system. 


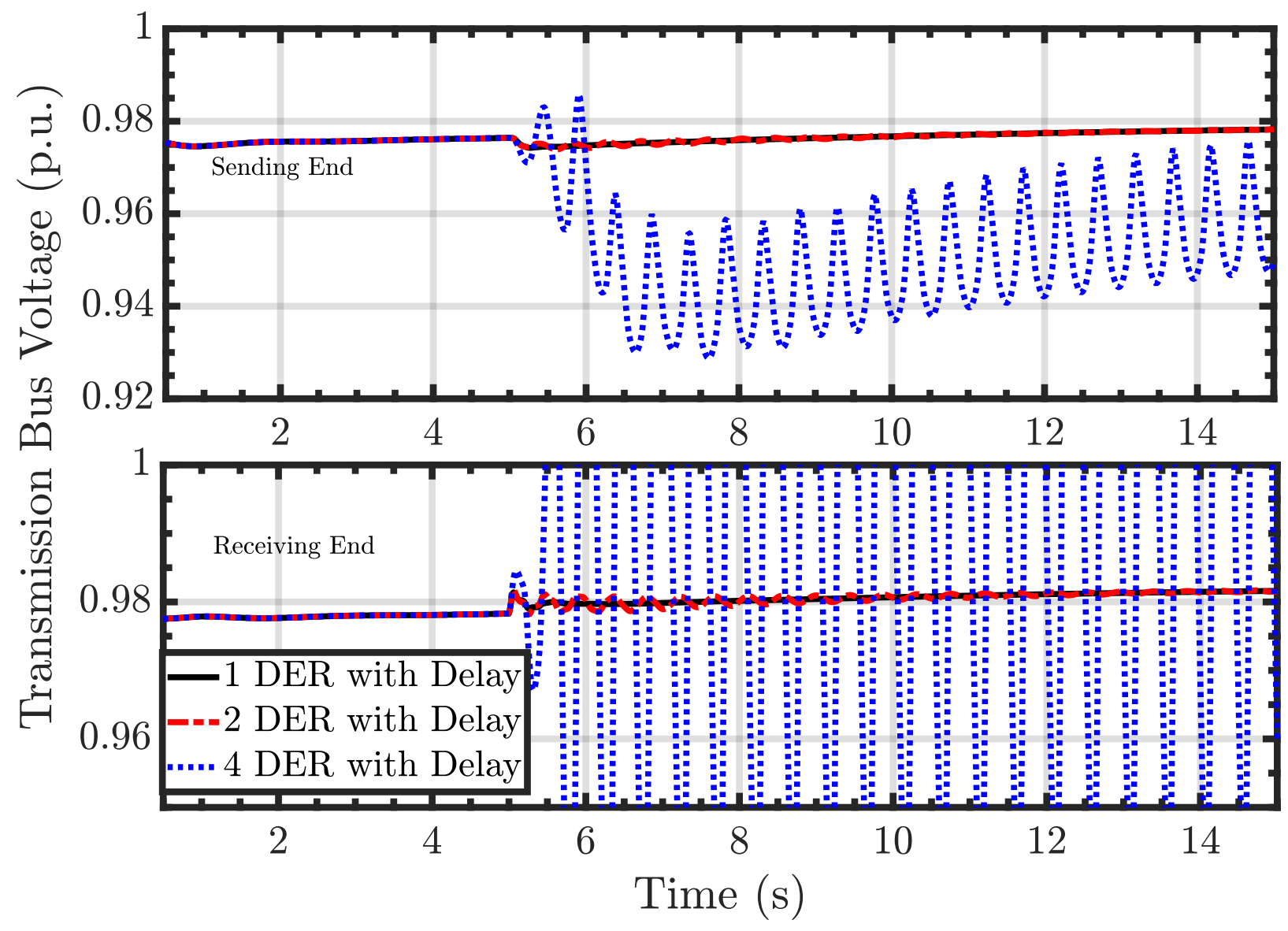

Figure 50 Tie-Line Bus Voltage Comparison.

Figure 51 compares the active and reactive power flowing toward the distribution system, respectively, from the bulk power grid, showing that the oscillations resulting from the reactive power exchange between the DERs and distribution system can lead to the oscillations in the power drawn by the distribution system from the transmission network. In addition, with reactive power support from the DERs, the reactive power consumed from the BES by the distribution system can be reduced; however, this condition might lead to increased consumption of active power by the voltage-dependent loads in the distribution system as the voltage in the system rises.

The impact of improper coordination of volt-VAR settings between multiple DERs within the distribution system was also observed in other BES variables, including the tie-line power flow, T\&D boundary bus active and reactive power flow, and the speed of BES generators. 


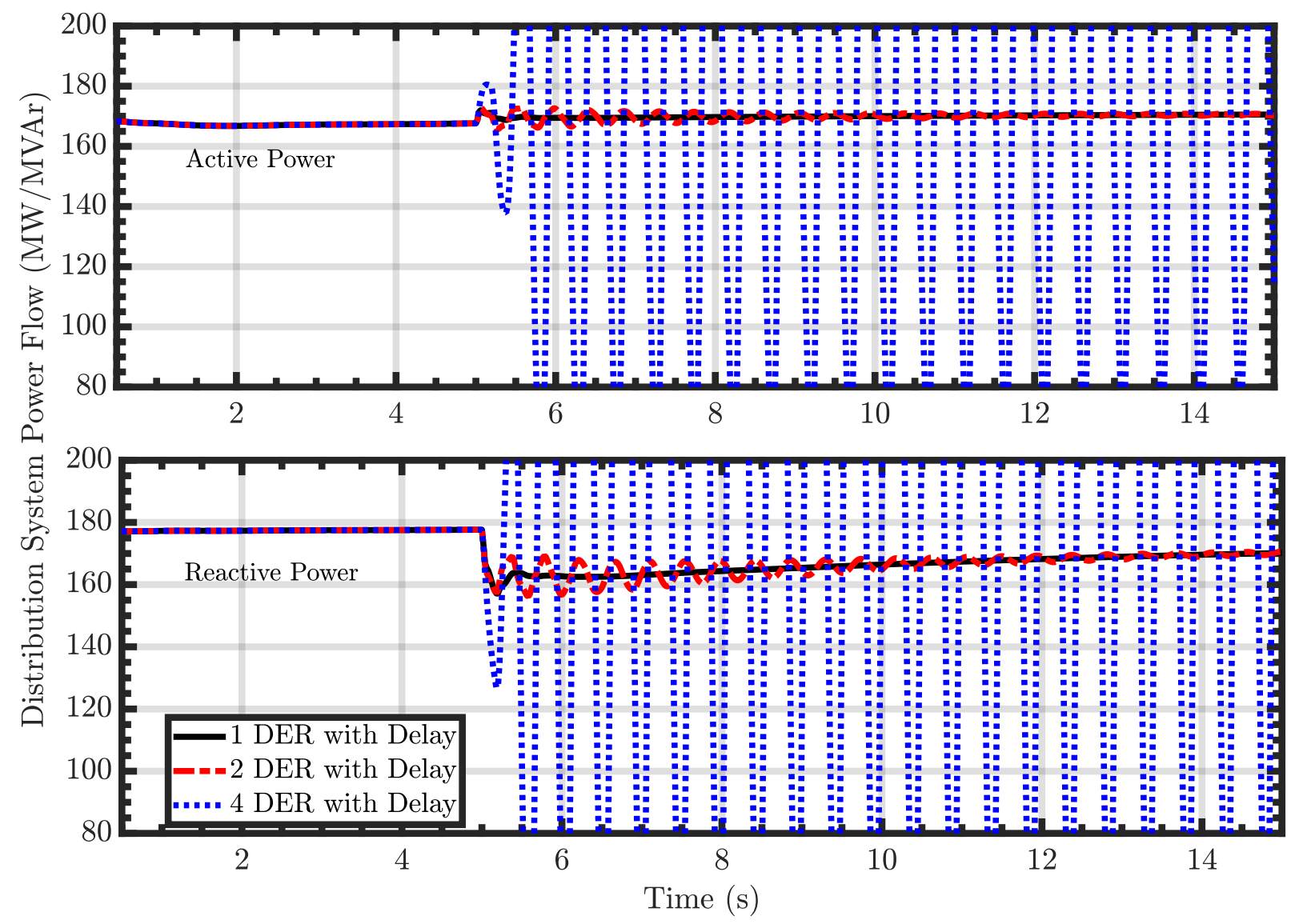

Figure 51 T\&D Boundary Bus Active and Reactive Power Flow Comparison.

\subsection{EFFECT OF DER REACTIVE POWER SUPPORT CAPABILITY TO ENHANCE BES VOLTAGE CONTROL DURING FAULT CONDITIONS}

This section addresses the effect of the reactive power support capability of DERs to enhance the voltage control and stability of the BES.

For the representative distribution system considered, the PV penetration level when compared to the distribution system load is close to $72 \%$. For the cases considered in this study, the penetration of PVs when compared to the overall BES load is close to $10 \%, 20 \%$, and $30 \%$, respectively, and close to $16 \%, 32 \%$, and $48 \%$, respectively, when compared to the load in Area 2 of the test power system (see Appendix B).

\subsubsection{Impact of DER without Reactive Power Support on BES Stability}

The following four scenarios are studied herein. Scenario 1 is the base case with zero DER penetration in the test system. Scenario 2, Scenario 3, and Scenario 4 each contain 16\%, $32 \%$, and $48 \%$ of DER penetration, respectively, compared to the total load in Area 2 . The power outputs of the generators in Area 1 are adjusted slightly to compensate for the changes in system 
losses as DER penetration in Area 2 increases. However, the Area 2 generators are taken off the grid to accommodate the increased penetration of DERs in Area 2. In cases 2, 3, and 4, the VoltVAR control functions in DERs are disabled. The objective of this case study is to consider the impact of legacy DER systems (without voltage regulation capability) on BES stability.

A three-phase, self-clearing bolted fault of 12 cycles is applied at the middle of one of the tie-lines connecting Area 1 and Area 2 at time $t=15$ seconds. Please note that of all the scenarios contemplated in this study, none of the DERs connected in the test distribution system trips as a result of frequency or voltage deviations.

Figure 52 shows the comparison of tie-line active power flow for the four cases under the fault condition. The result indicates that as the DER penetration increases, the tie-line active power flow oscillations (both in magnitude and duration) increase following the fault. This result suggests the need for better damping controllers in the BES - as the DER penetration level increases, the number of synchronous machines with power system stabilizers and damping controllers decrease.

Figure 53 delineates the sending- and receiving-end voltages of the tie-line for the four cases. The figure shows that the voltage sag in the receiving end of the tie-line increases as DER penetration increases. The voltage oscillations in both the sending and receiving ends of the tieline increase as well with higher DER penetration scenarios. The increase in voltage sag on the receiving end can be attributed to the reduced number of reactive power reserves in the form of synchronous machines in Area 2.

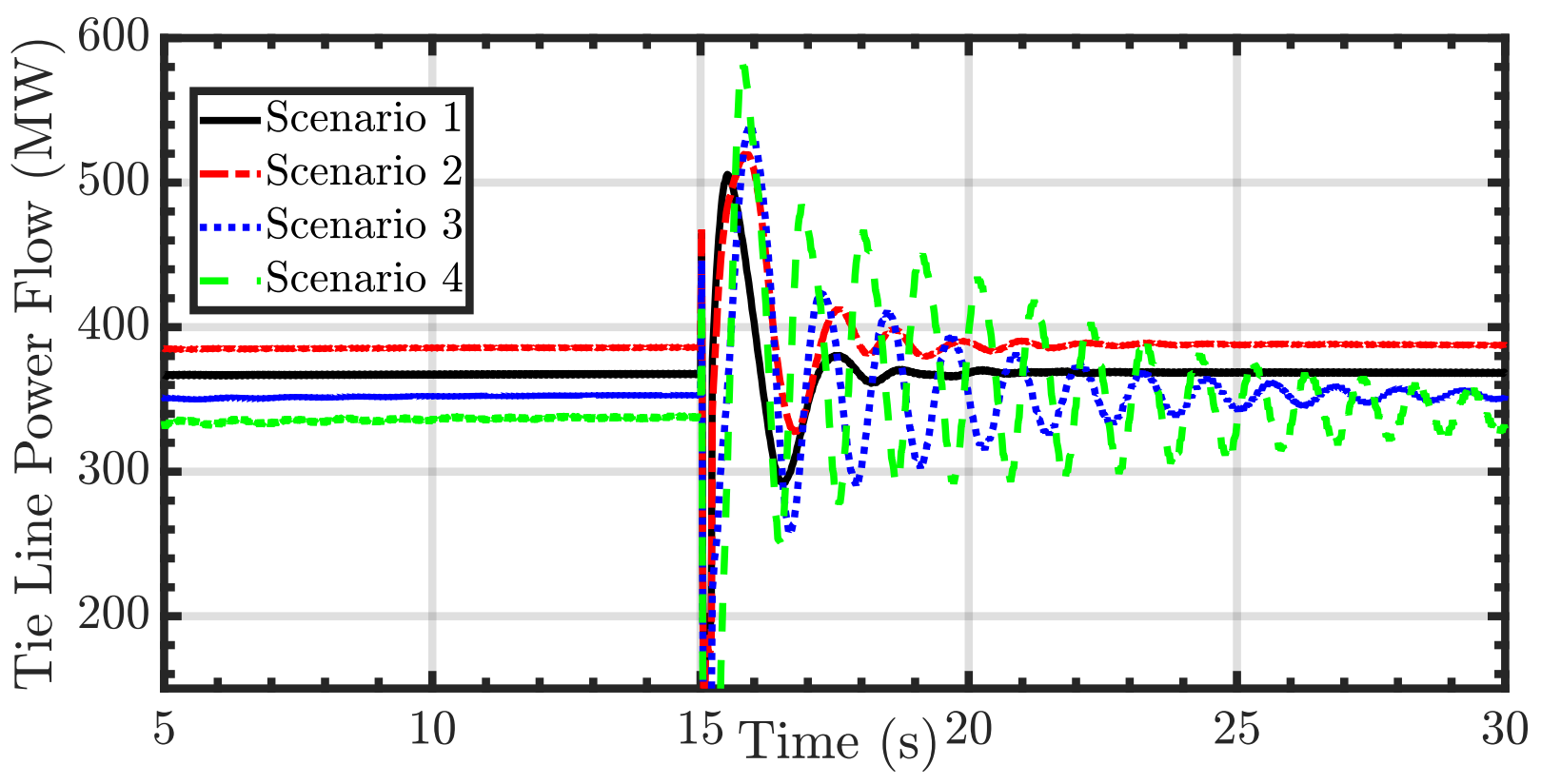

Figure 52 Comparison of Tie-Line Active Power Flow. 


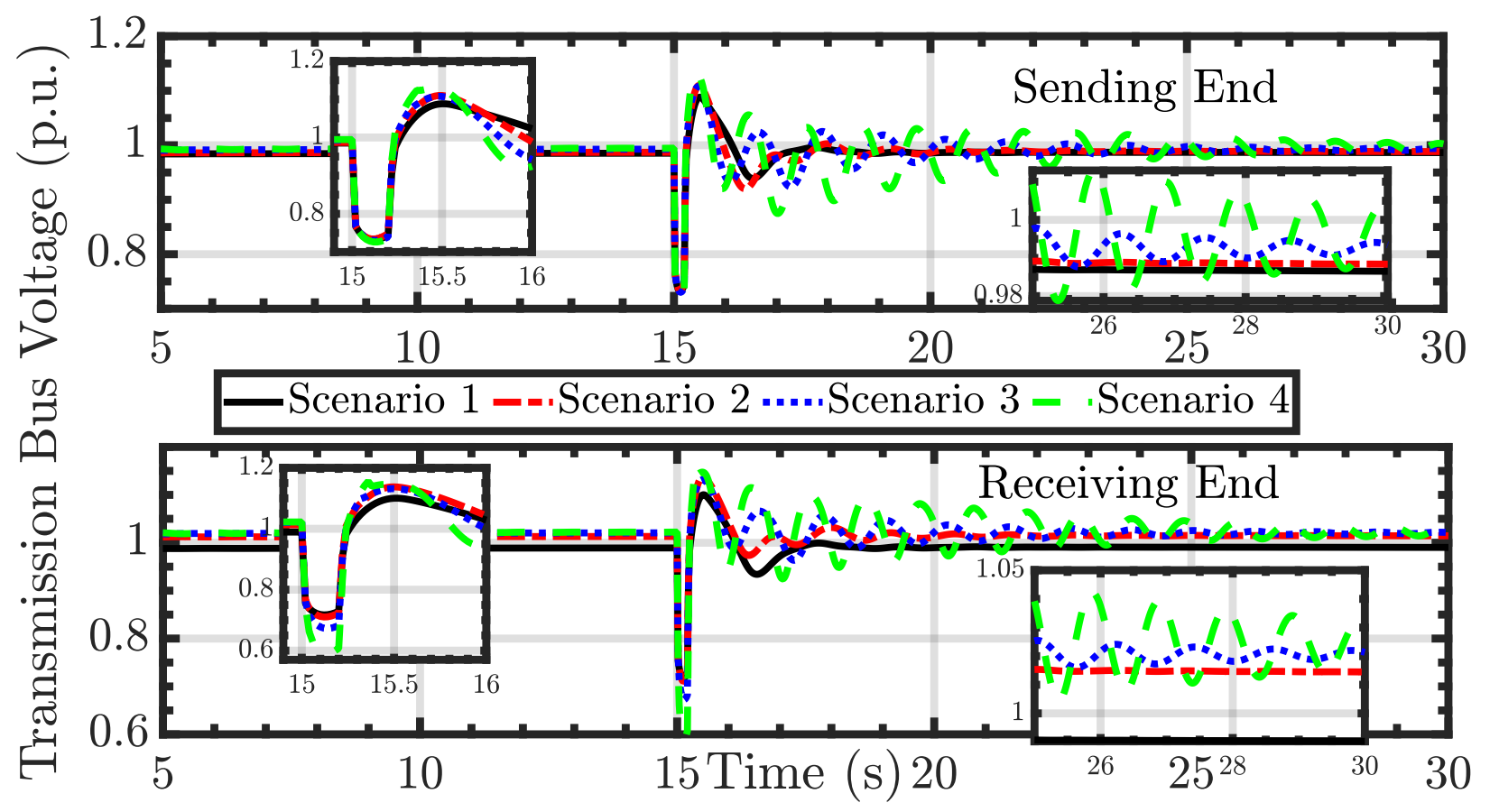

Figure 53 Comparison between Tie-Line Sending-End and Receiving-End Voltages.

Figure 54 compares the generator rotor speed for the different cases considered. As DER penetration increases, the speed deviation from nominal speed increases following the fault in the tie line. This result can be attributed to the reduced inertia in the system as DER penetration increases. Also note that at higher penetration levels of DERs, the rate of frequency recovery back to the nominal value is larger than in the base case and cases with lower DER penetration (see zoomed-in plot for G3 in Figure 54). Also note that the rotor speed oscillations are higher for generators in Area 2 as compared to generators in Area 1, which results from the reduced inertia in Area 2 as compared to Area 1. 


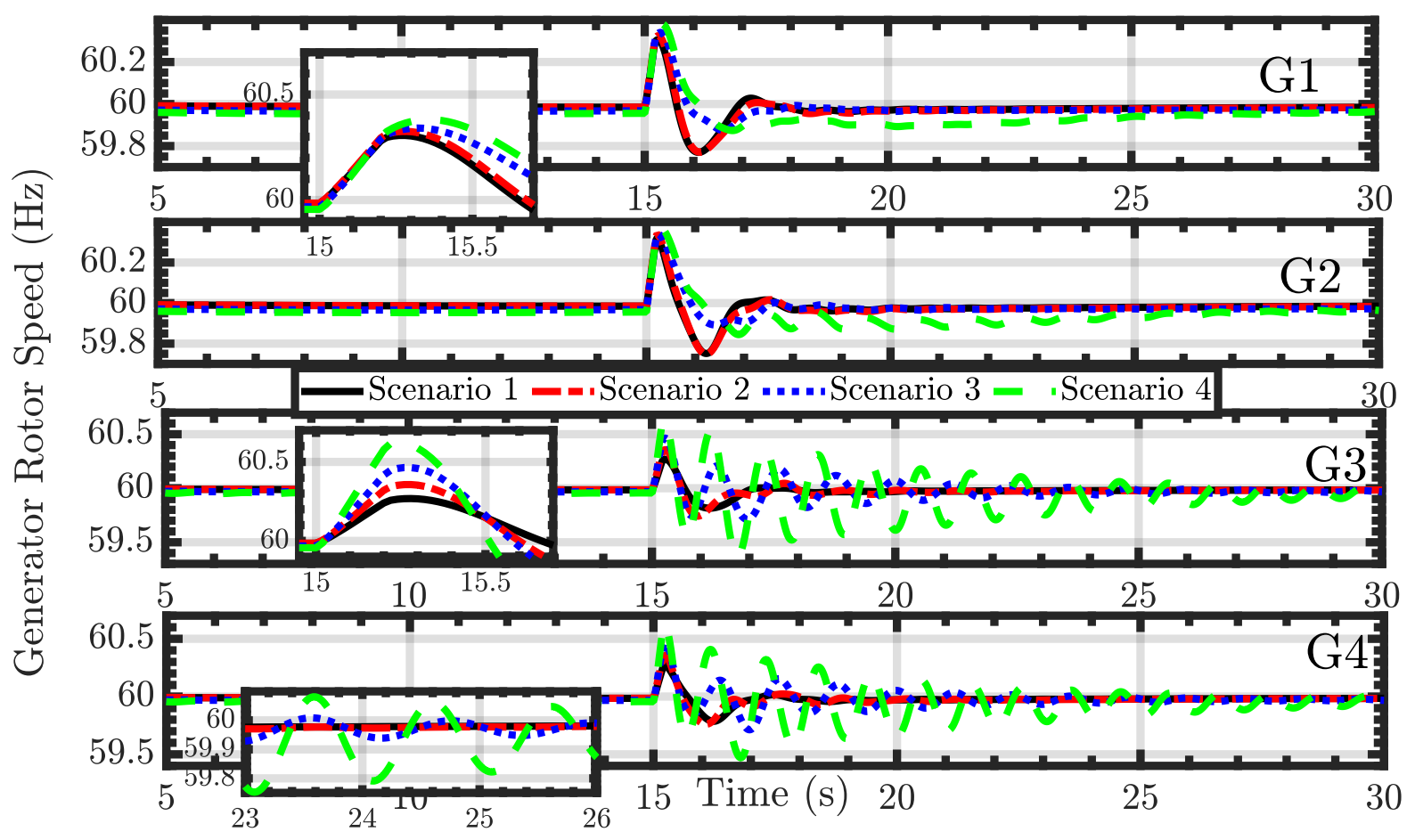

Figure 54 Comparison of Generator Rotor Speed.

\subsubsection{Effect of DERs with Reactive Power Support to Enhance BES Stability}

This section considers the impact of DERs that have enabled their reactive power support capability on BES stability.

Four scenarios with various Volt-VAR slope settings are created to facilitate the studies, while the penetration level of DERs is kept constant at $32 \%$ for all four cases when compared with the load in Area 2. Specifically, Scenario 1 corresponds to the base case with no reactive power support from DERs in the test system. Scenario 2 corresponds to the case where the DERs in Area 2 are characterized as providing mild Volt-VAR support. In Scenario 3, the DERs in Area 2 have normal Volt-VAR support settings as per the slope of the Volt-VAR curve used. Scenario 4 corresponds to an operational condition where the DERs in Area 2 have steep VoltVAR support settings. The specific settings of mild, normal, and steep Volt-VAR control levels are introduced in Section 4.1.2. In Scenarios 2, 3, and 4, the DERs have an active power priority mode, which here means that the DERs will try to maintain their active power output at the reference set point and will do so even by compromising the reactive power output.

A three-phase, self-clearing bolted fault of 12 cycles is applied at the middle of one of the tie-lines connecting Areas 1 and 2 at time $t=15$ seconds. Please note that none of the DERs connected in the distribution system trip offline owing to voltage or frequency deviations in this case study. 
Figure 55 shows the comparison of tie-line active power flow for the four scenarios considered and indicates that the slope of the Volt-VAR curve implemented in the DERs has a direct impact on the damping of the inter-area tie-line power oscillations. The figure shows that with the steep Volt-VAR curve (Scenario 4), the first swing following the fault clearance is minimized. DERs with mild (Scenario 2) and normal (Scenario 3) Volt-VAR controls also reduce the magnitude of the first swing following the fault. However, the steep Volt-VAR curve has less damping when compared to mild and normal Volt-VAR curves. It is evident that the damping of the oscillations is enhanced with the use of mild and normal Volt-VAR curves compared to both the no-Volt-VAR support and the steep Volt-VAR support scenarios. This result indicates that there may be an optimal setting for Volt-VAR control that can have the most beneficial impact on the system's oscillation damping.

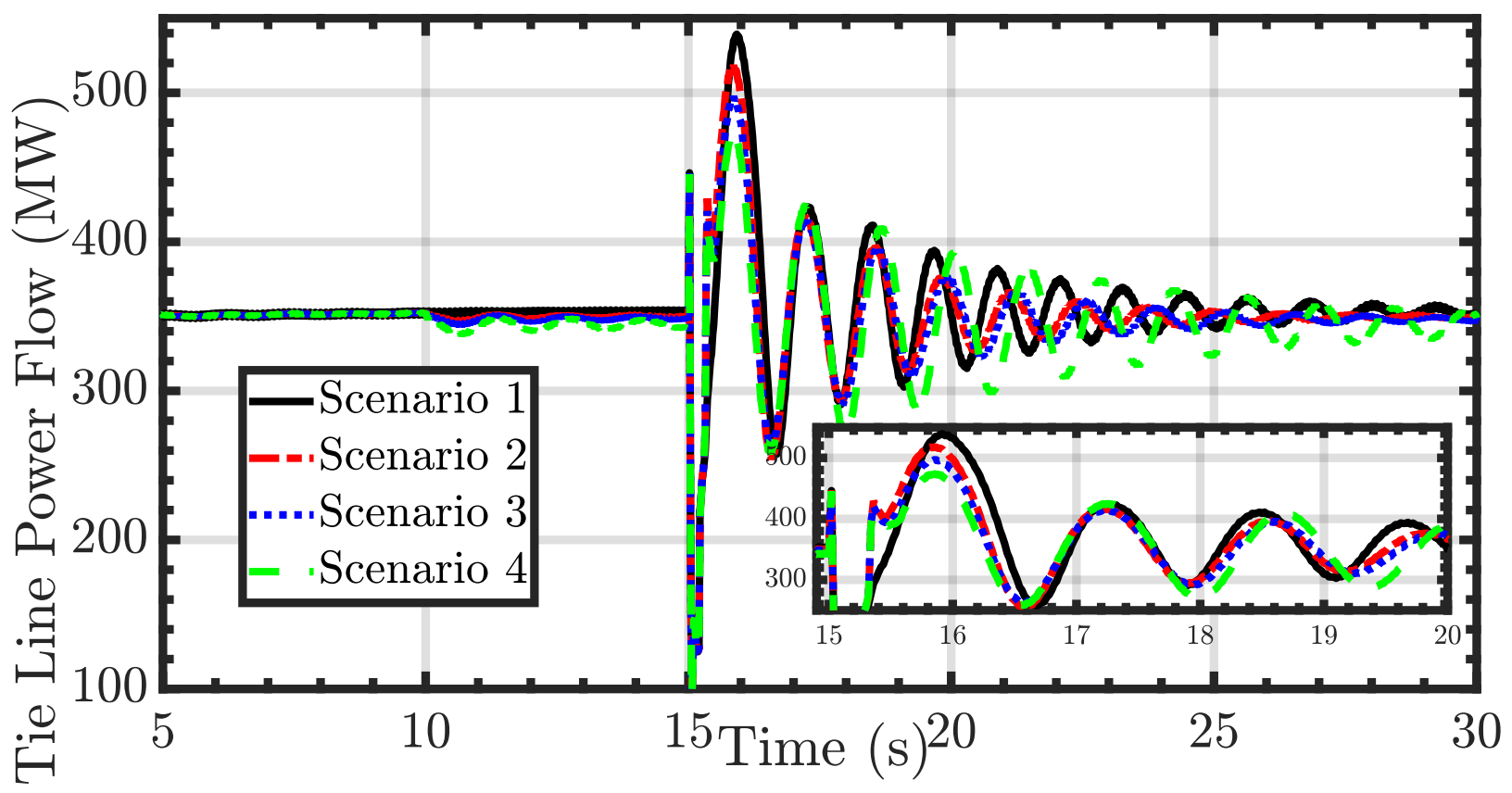

Figure 55 Comparison of Tie-Line Active Power Flow.

Figure 56 delineates the transmission bus sending- and receiving-end voltage for the different scenarios considered; the voltage sag in the receiving-end and sending-end bus of the transmission system remains unchanged for different Volt-VAR settings. This result occurs because of the P-priority setting implemented in the DERs, which limits the reactive power injection from DERs during the fault condition (see the plot from the PV reactive power output). It is evident that following the fault clearance, the tie-line voltage settles back to the pre-fault value sooner in the scenarios featuring voltage support from DERs. This result shows that the reactive power support from DERs can help the BES maintain voltage stability and can compensate for the reactive power reserve loss that results from the loss of synchronous machines. It is evident that better damping and recovery of the BES voltage are observed for the 
scenarios with mild and normal Volt-VAR settings as opposed to scenarios with steep volt-VAR settings.

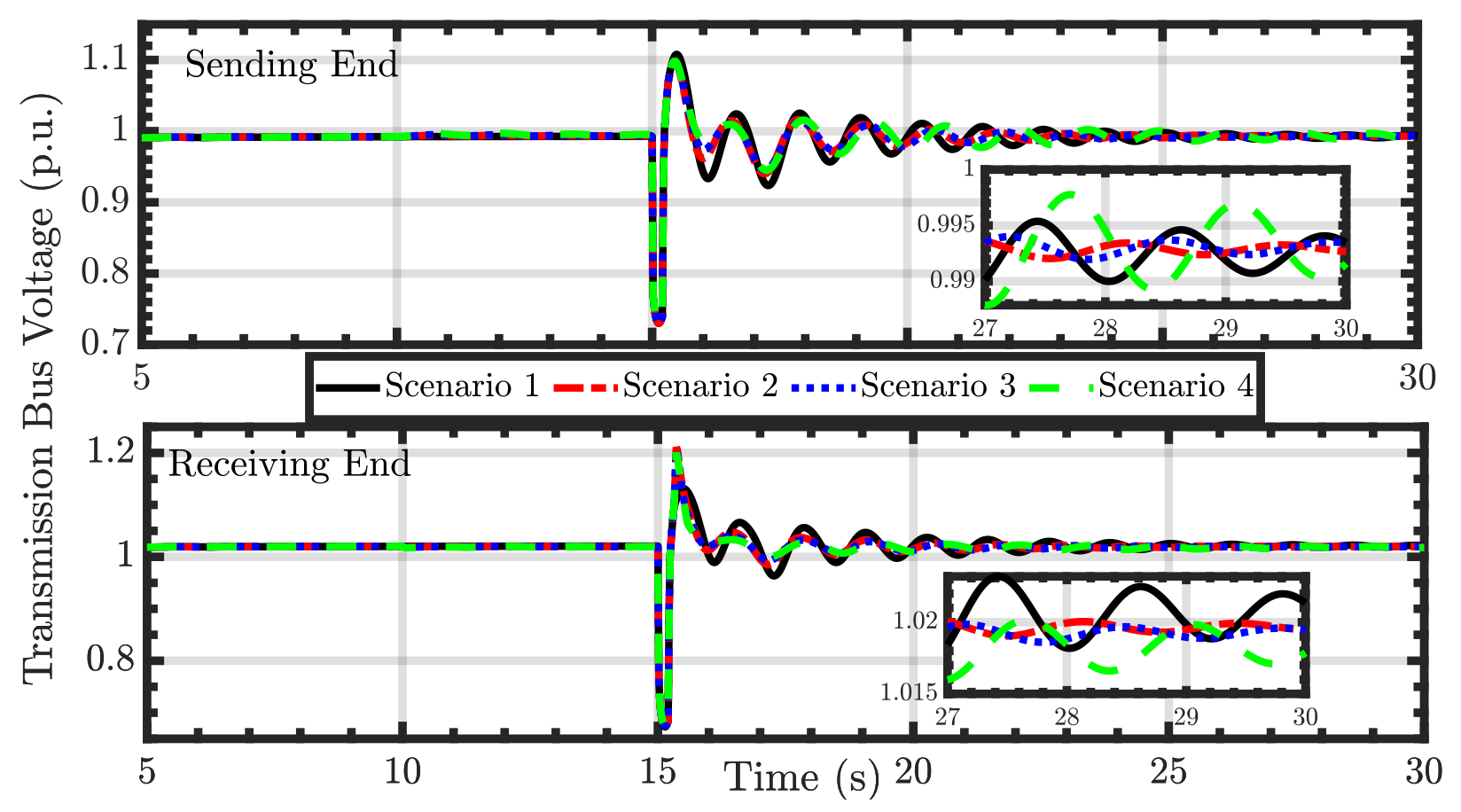

Figure 56 Comparison between Tie-Lines' Sending-End and Receiving-End Voltages.

Figure 57 compares the generator rotor speed for the different scenarios being considered. With reactive power support from the DERs, the oscillation and deviation of the generator rotor speed are reduced (for mild and normal Volt-VAR curves). This result can be associated with there being less oscillation in the transmission bus voltages, which leads to less variation in the power consumed by constant impedance-type lumped loads connected at the transmission bus, thus reducing the oscillation in the generator rotor speed. Also note that the rotor speed oscillations are higher for generators in Area 2 as compared to generators in Area 1, which is attributable to the reduced effective inertia in Area 2 as compared to Area 1 with the addition of DERs in Area 2. For steep Volt-VAR curves as the reactive power injected from the DERs is more sensitive to the voltage variation, the damping of the generator speed oscillations has deteriorated. 


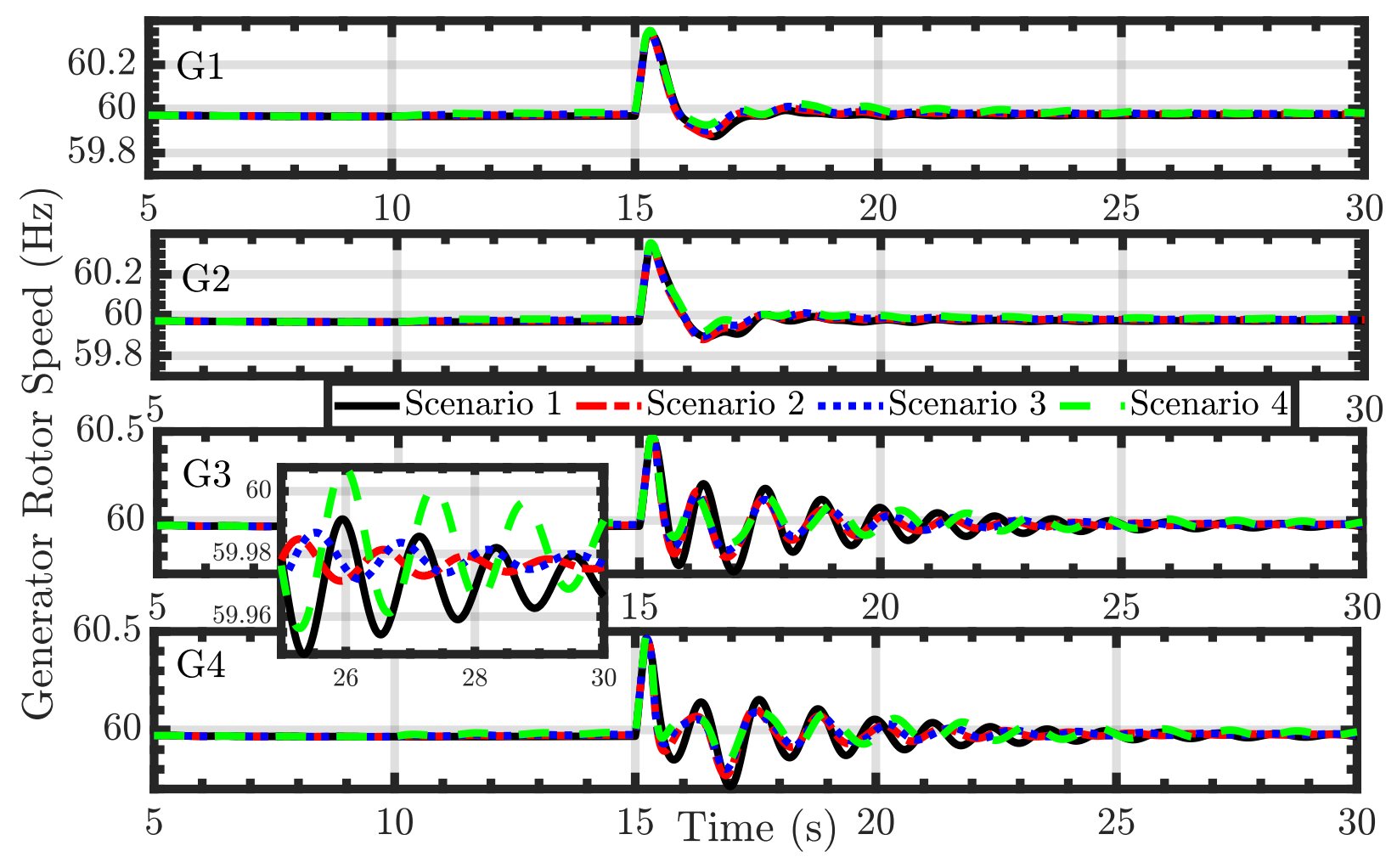

Figure 57 Comparison of Generator Rotor Speed.

Figure 58 shows the PV PCC voltage for the various scenarios being considered. As no reactive power is injected during the fault period by the DERs owing to the priority for active power, the PCC voltage during the sag is the same for all scenarios. However, once the fault is cleared, the active power component of current is recovered back to the nominal range, and a surge of reactive power is injected by the DERs (see the PV reactive power output), causing the PCC voltage to spike up. The PV PCC voltage is observed to be better dampened for the scenarios with Volt-VAR support when compared to the base scenario without Volt-VAR support. 


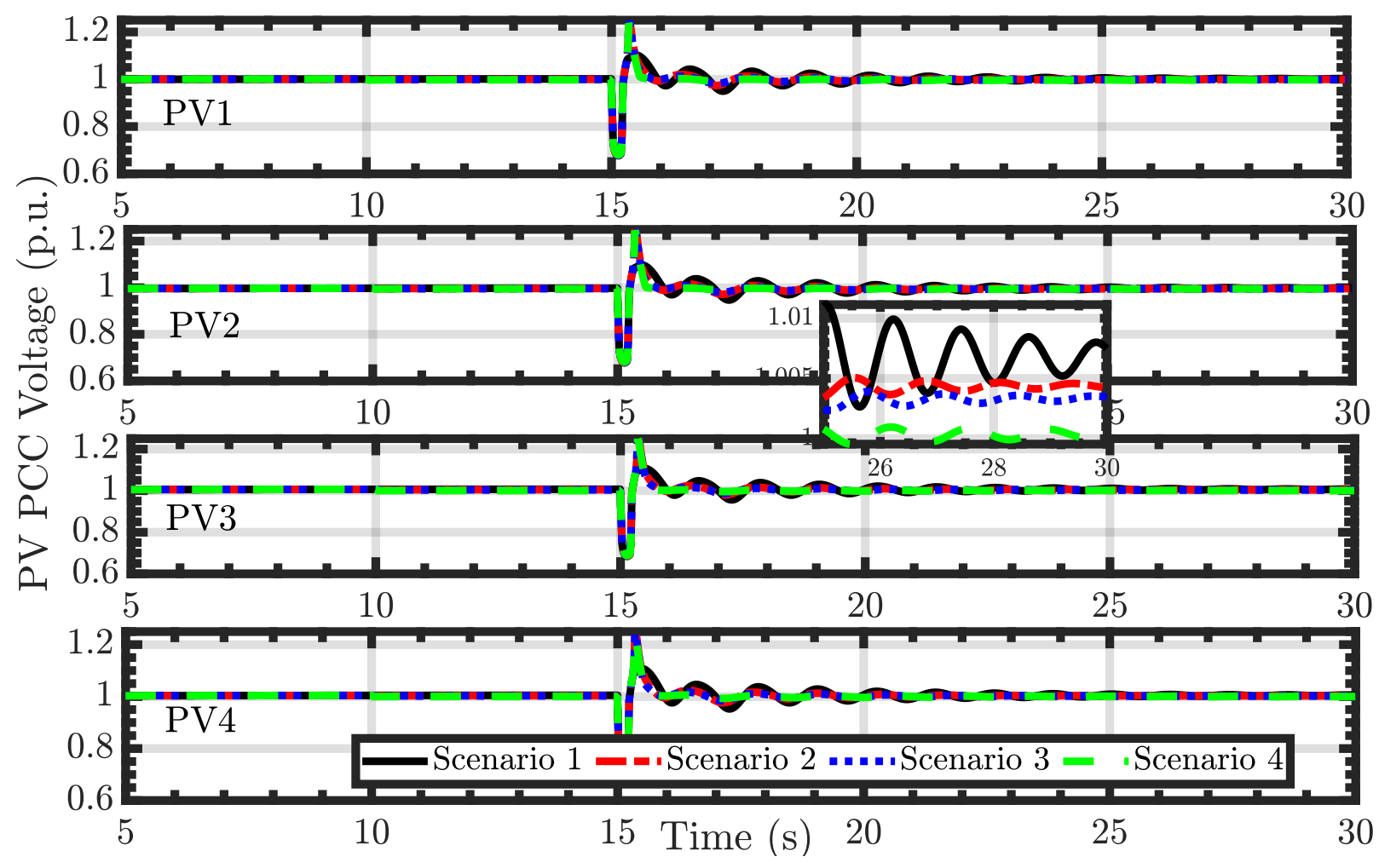

Figure 58 Comparison of PV PCC Voltages.

Figure 59 shows the reactive power output of the PV systems in the distribution feeder. The reactive power output of the PV system is maintained close to zero with few fluctuations as a result of the disturbance in the system at 15 seconds. Because of the PV system's priority for active power, the PV systems do not inject any reactive power into the distribution system during the period of fault. However, as the fault clears, the active power component of the DERs current returns within the nominal band and with margin available for reactive current injection. At that point, a large surge of reactive current is injected into the distribution system after around 15.4 seconds. The oscillations in the reactive power output are directly proportional to the slope of the Volt-VAR curve used - the steeper the Volt-VAR curve, the more oscillations.

Figure 60 shows the active power output of the PV systems for the different scenarios. The PV systems can maintain a constant power output in steady state and can quickly restore their power output to the pre-disturbance level following the fault. 


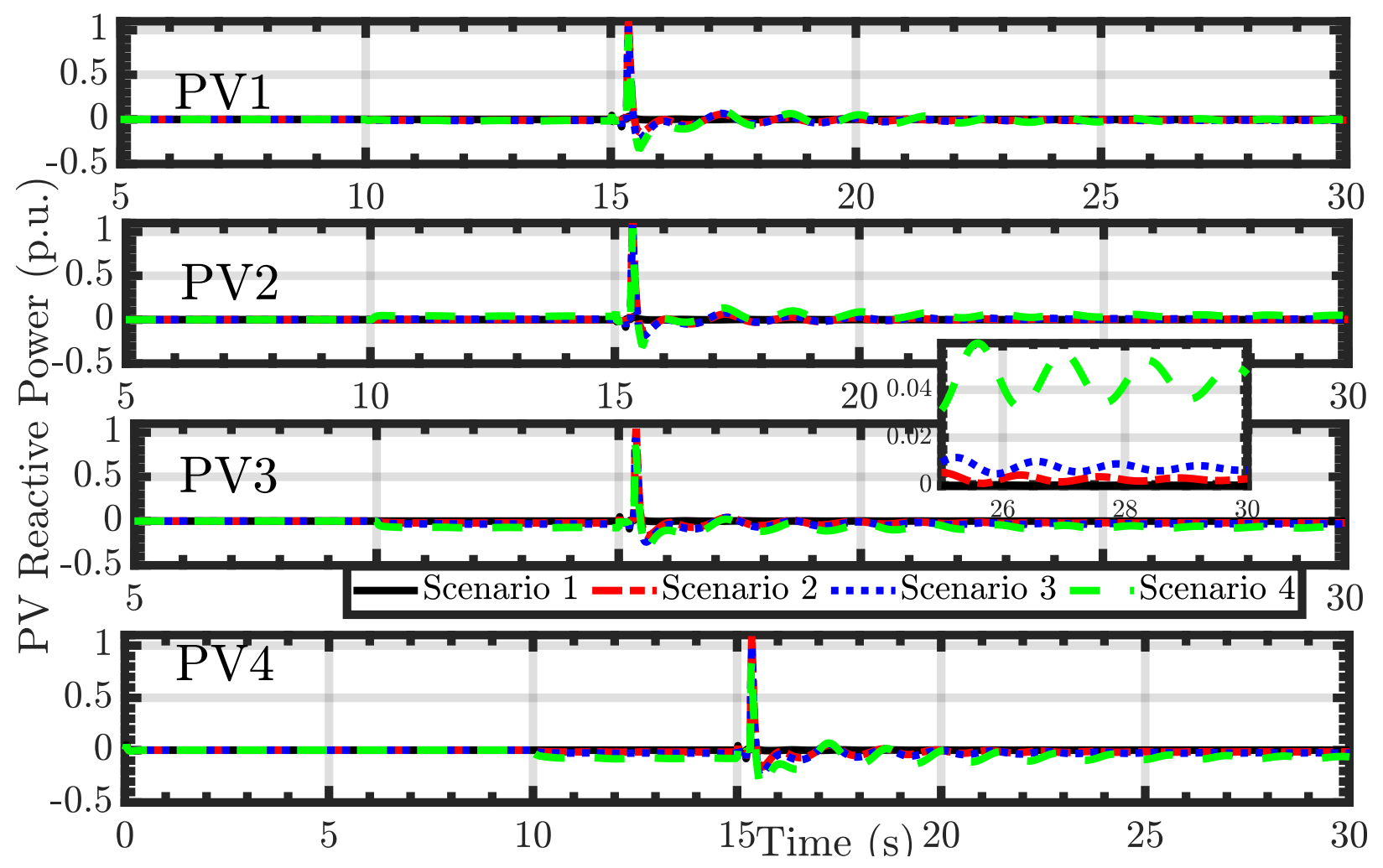

Figure 59 Comparison of PV PCC Reactive Power Output. 


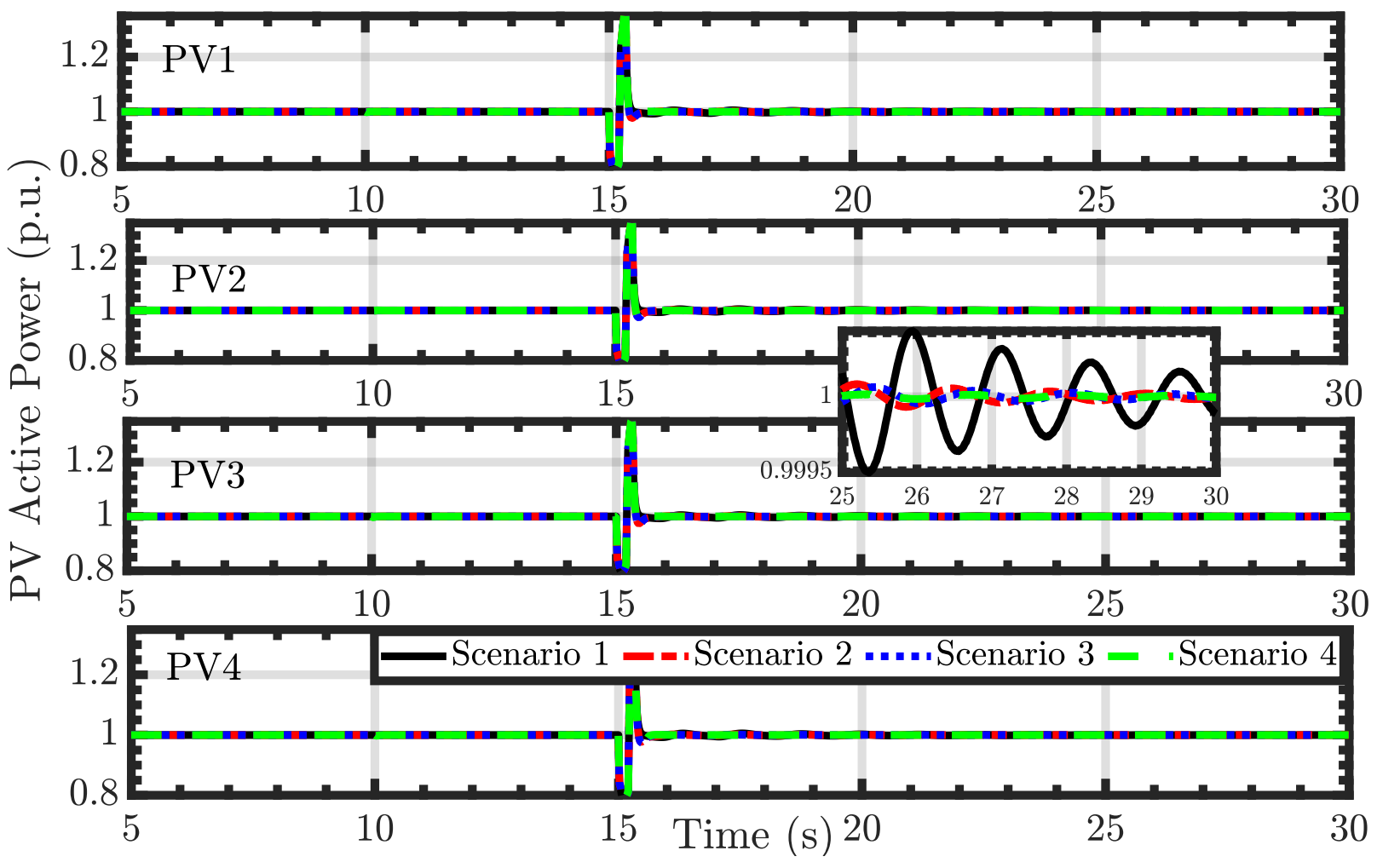

Figure 60 Comparison of PV PCC Active Power Output. 


\section{IMPACT OF DER PENETRATION ON DISTRIBUTION SYSTEM PROTECTION COORDINATION}

Overhead distribution systems are common in rural areas, while underground distribution systems are widely adopted in urban areas. Regardless of the variations in distribution system composition, overcurrent protection is the most widely adopted protection scheme for distribution systems. It relies on measured currents to distinguish faults from nominal load currents. The devices used most often in distribution system protection include overcurrent relays, reclosers, sectionalizers, and fuses. These devices, except sectionalizers, all implement a set of time/current curves, which have a time-inverse characteristic and provide different operation times, depending on the fault current level, that is, the larger the fault current, the shorter the operation time will be.

The basic requirements for a protection device include selectivity, sensitivity, operating time, and stability. The relay settings are very important in ensuring selectivity and sensitivity. Traditionally, because of the passive nature of distribution networks, the power flow in the distribution network is one-way (i.e., from the distribution substation to customers). When a fault occurs in the distribution system, the main source feeding the fault is the transmission system. Conventional protection devices are set up and coordinated on the basis of one-way power flow, ensuring that the upstream protection device closest to the fault reacts first to clear the fault. Each relay provides backup to the next downstream relay with a time delay. An upstream relay will not react to the fault current unless its downstream relay fails to react within the setting time.

The conventional distribution system protection scheme faces challenges with the high penetration of DERs. Although the fault current contribution from each DER is limited [44], a high penetration of DERs can contribute a considerable amount of fault current. These sources can change the fault current distribution and magnitude and cause new problems for the operation of existing overcurrent protection schemes [45]. In addition, the DERs installed in the distribution system have their own protection relays in place with specific protection schemes, such as anti-islanding [44]-[45], detecting abnormal voltage/frequency, and tripping, with different time responses according to different voltage/frequency deviations [44]-[45]. Poor coordination between the DER relays' protection schemes and conventional feeder protection devices may lead to nuisance fuse blowing, reclosing out of synchronism, and sectionalizer miscount, etc.

This chapter attempts to demonstrate some of the emerging protection issues resulting from high DER penetration including increased fault current, sympathetic tripping, nuisance tripping, and desensitized feeder head protection relays. First, we describe the distribution system used for the protection studies. 


\subsection{DESCRIPTION OF THE DISTRIBUTION SYSTEM FOR PROTECTION STUDIES}

The schematic diagram of the distribution system modeled for protection studies is shown in Figure 61. A distribution system with two IEEE 34-node feeders connected to the same bus has been developed using the DIgSILENT PowerFactory. The two test feeders are identical in terms of their topology, protection devices and their configurations, loading, and so on. The only difference between these two feeders is as follows: Feeder 1 (the upper feeder) has a synchronous generator as a distributed generation connected to its node 808 , which offsets $500 \mathrm{~kW}$ from a total load of 2,067 kW. As a result, Feeder 1 draws $1,539 \mathrm{~kW}$ from the upstream transmission system, and Feeder 2 (the lower feeder) draws 2,067 kW from the upstream transmission system. Each feeder is protected by a circuit breaker at the feeder head, and each lateral is protected by a fuse. Characteristics of the inverse time current of the recloser and fuses used in the test distribution system are shown in Figure 62.

Table 9 provides the rating of each fuse used in the distribution feeder.

Table 9 Rating of Each Fuse in the Distribution Feeder.

\begin{tabular}{lc}
\hline Fuse & Current Rating \\
\hline F1 & $80 \mathrm{~A}$ \\
F2 & $63 \mathrm{~A}$ \\
F3 & $50 \mathrm{~A}$ \\
F4 & $50 \mathrm{~A}$ \\
F5 & $32 \mathrm{~A}$ \\
F6 & $40 \mathrm{~A}$ \\
F7 & $25 \mathrm{~A}$ \\
F8 & $25 \mathrm{~A}$ \\
F9 & $40 \mathrm{~A}$ \\
F10 & $40 \mathrm{~A}$ \\
F11 & $25 \mathrm{~A}$ \\
F12 & $100 \mathrm{~A}$ \\
\hline
\end{tabular}




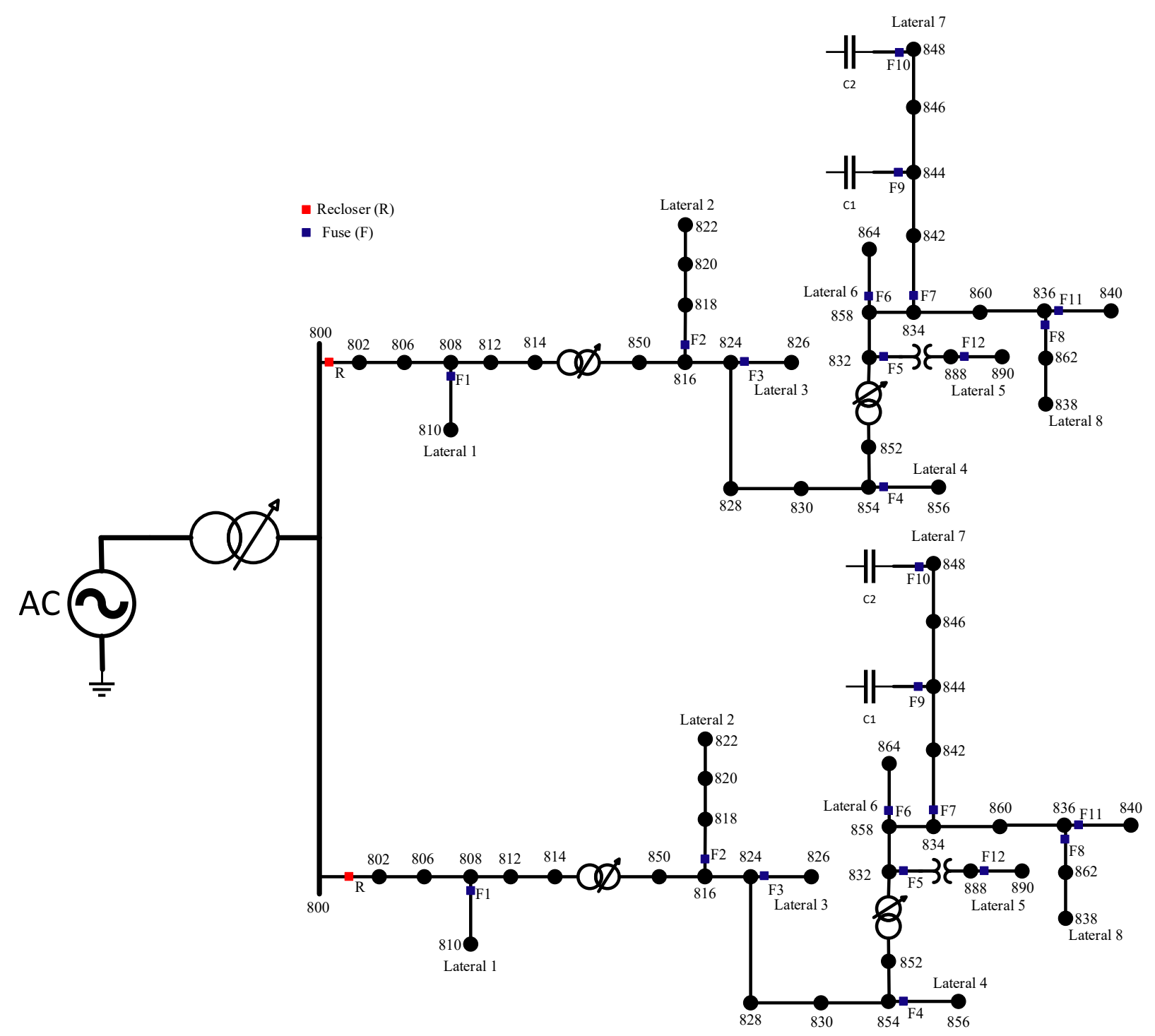

Figure 61 Schematic Diagram of the Distribution System for Protection Studies. 


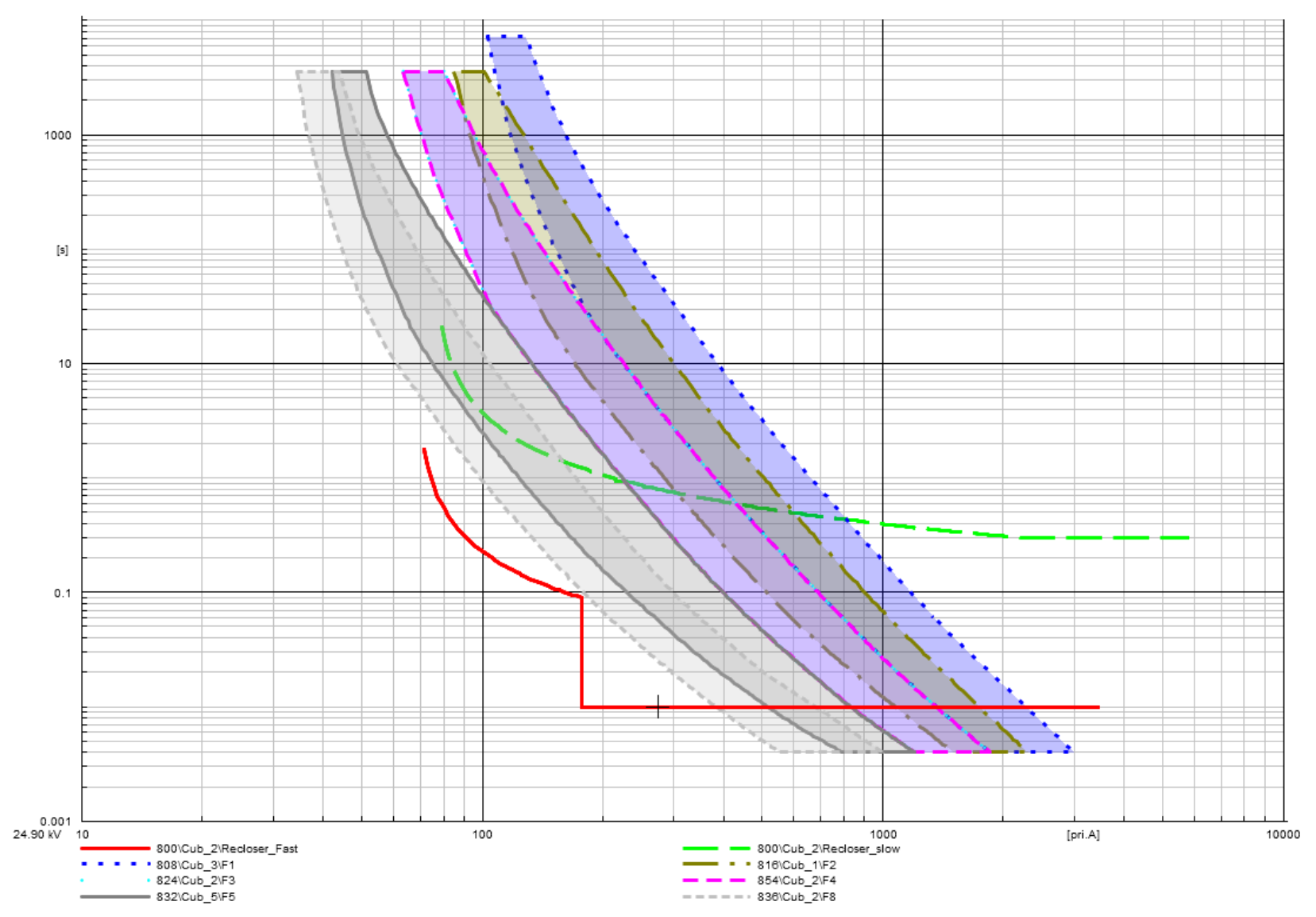

Figure 62 Inverse Time Current Characteristics of the Recloser and Fuses Used in the Test Distribution System.

\subsection{INCREASED FAULT CURRENT}

High penetration of DERs on a distribution feeder will contribute to a higher magnitude of fault current following a fault on the feeder. To demonstrate the increase in fault current with the addition of DGs in the feeder, we studied a short circuit analysis with $0+\mathrm{j} 0$ fault impedance at node 890. Table 10 shows that as PV penetration in the distribution feeder grows, the fault current by the fuse close to the fault (F12) increases; however, at the same time, it reduces the current passing through the recloser. This reduction of current passing through the recloser can desensitize the relay, depending on the location of DGs, location of the fault, and source and line impedances. (More discussion of the "desensitizing of protection devices follows in following subsections.) In addition, it is interesting to note that the aggregate fault current contribution from DGs on a single circuit may affect fault current on other circuits fed from the same substation bus, which can at times lead to sympathetic tripping; this phenomenon is discussed in the Section 5.3.

These changes in the fault current levels can also make it necessary to review the existing protection coordination currently implemented in the distribution network. 
Table 10 Fault Current Distribution with Varying PV Penetration Levels.

\begin{tabular}{lllll}
\hline \multicolumn{1}{c}{ PV Location } & $\begin{array}{c}\text { Steady State } \\
\text { Fuse Current } \\
\text { (A) }\end{array}$ & $\begin{array}{c}\text { Steady State } \\
\text { Recloser } \\
\text { Current (A) }\end{array}$ & $\begin{array}{c}\text { Fault Fuse } \\
\text { Current (A) }\end{array}$ & $\begin{array}{c}\text { Fault Recloser } \\
\text { Current (A) }\end{array}$ \\
\hline No PV & & & & \\
PV at 888 & 13.207 & 55.15 & 72.881 & 106.258 \\
PV at 888, 832 & 12.937 & 52.099 & 73.904 & 104.964 \\
PV at 888, 832, 854 & 12.761 & 49.313 & 74.432 & 103.45 \\
PV at 888, 832, 854, 848 & 12.641 & 46.792 & 74.863 & 101.773 \\
PV at 888, 832, 854, 848, 890 & 12.479 & 44.086 & 75.307 & 100.474 \\
PV at 888, 832, 854, 848, 890, 834 & 12.325 & 41.452 & 75.735 & 99.227 \\
PV at 888, 832, 854, 848, 890, 834, 850 & 12.181 & 38.883 & 76.321 & 97.602 \\
& 12.112 & 36.631 & 76.612 & 95.935 \\
\hline
\end{tabular}

\subsection{SYMPATHETIC TRIPPING}

When a fault occurs on a distribution feeder, the aggregate fault current's contribution from DERs on the adjacent feeder may cause sympathetic tripping of the circuit breaker of the adjacent feeder. This outcome occurs when the aggregate fault's contribution from DGs on a single circuit may affect the fault current on other circuits fed from the same bus [44]-[45]. Figure 63 shows how sympathetic tripping can occur when the DER unit feeds a fault on a neighboring feeder (Feeder 2). In this case, the fault current supplied by the DER in Feeder 1 (left) can exceed the limit of the overcurrent protection of the circuit breaker and cause it to trip.

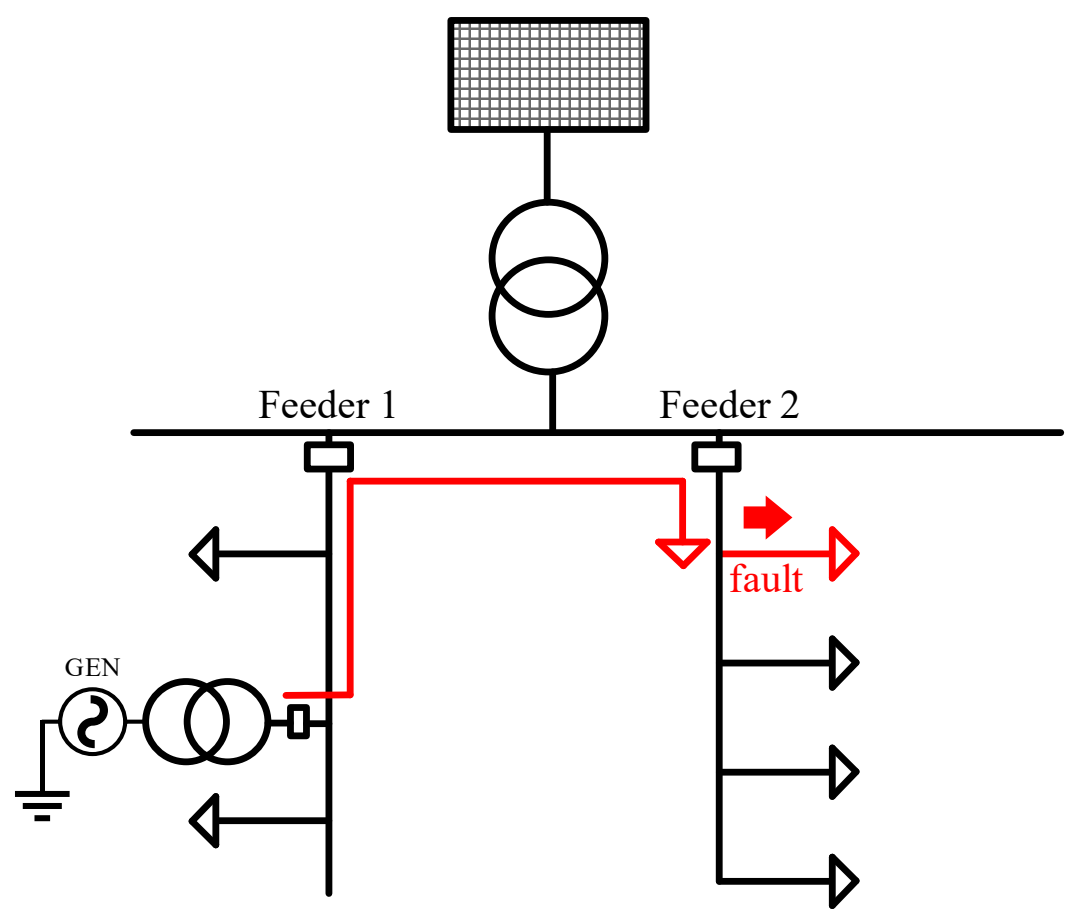

Figure 63 Illustration of Sympathetic Tripping. 
The sympathetic tripping is demonstrated on the test distribution system shown in Figure 61. A three-phase, bolted short-circuit fault is applied at 5 seconds on node 806 of Feeder 2 (down), which lasts for 0.75 seconds. Even though the fault is on Feeder 2, the recloser at the head of Feeder 1 (up) opens as the distributed generation in Feeder 1 contributes to the fault.

The recloser at the head of both feeders is operated in the fuse-saving mode, that is, as soon as the reclosers detect an increased amount of current, they open the feeder completely and allow the fault to clear itself without letting the fuse blow. Figure 62 shows the time-current curves of the recloser used at the feeder head.

The occurrence of sympathetic tripping depends on factors like the location of the fault on the faulted feeder (a fault on the end of an adjacent feeder may not be an issue as opposed to a fault close to the substation) and the type of DERs on the healthy feeder (e.g., inverter-based DERs may not contribute as large a fault current as synchronous generator-based DERs may do).

Figure 64 shows how having a distributed generation in a feeder can affect coordination of distribution feeder protection. In the example considered, the protection setup in the system is structured in such a way that the fault current generally comes in through the substation from the upstream transmission system and is the only source that feeds into the fault. However, with the addition of the DG on Feeder 2, there will be multiple sources that can feed into the fault and hence affect the protection coordination of the distribution system. Not all DGs contribute a large enough magnitude of fault current to significantly affect the protection coordination; however, synchronous generation-based DGs can. Inverter-based DERs, even though they are of the same rating as synchronous machine-based DGs, cannot provide a comparable magnitude of fault current (they are generally limited to a maximum of 1.2 times the rated current) to cause sympathetic tripping. Figure 64 shows the recloser status of Feeder 1, which is a healthy feeder. Nevertheless, when a DG is present in Feeder 1, it contributes to the fault in Feeder 2, causing the recloser in Feeder 1 to operate and thus affecting consumers tied in to the healthy feeder as well. 


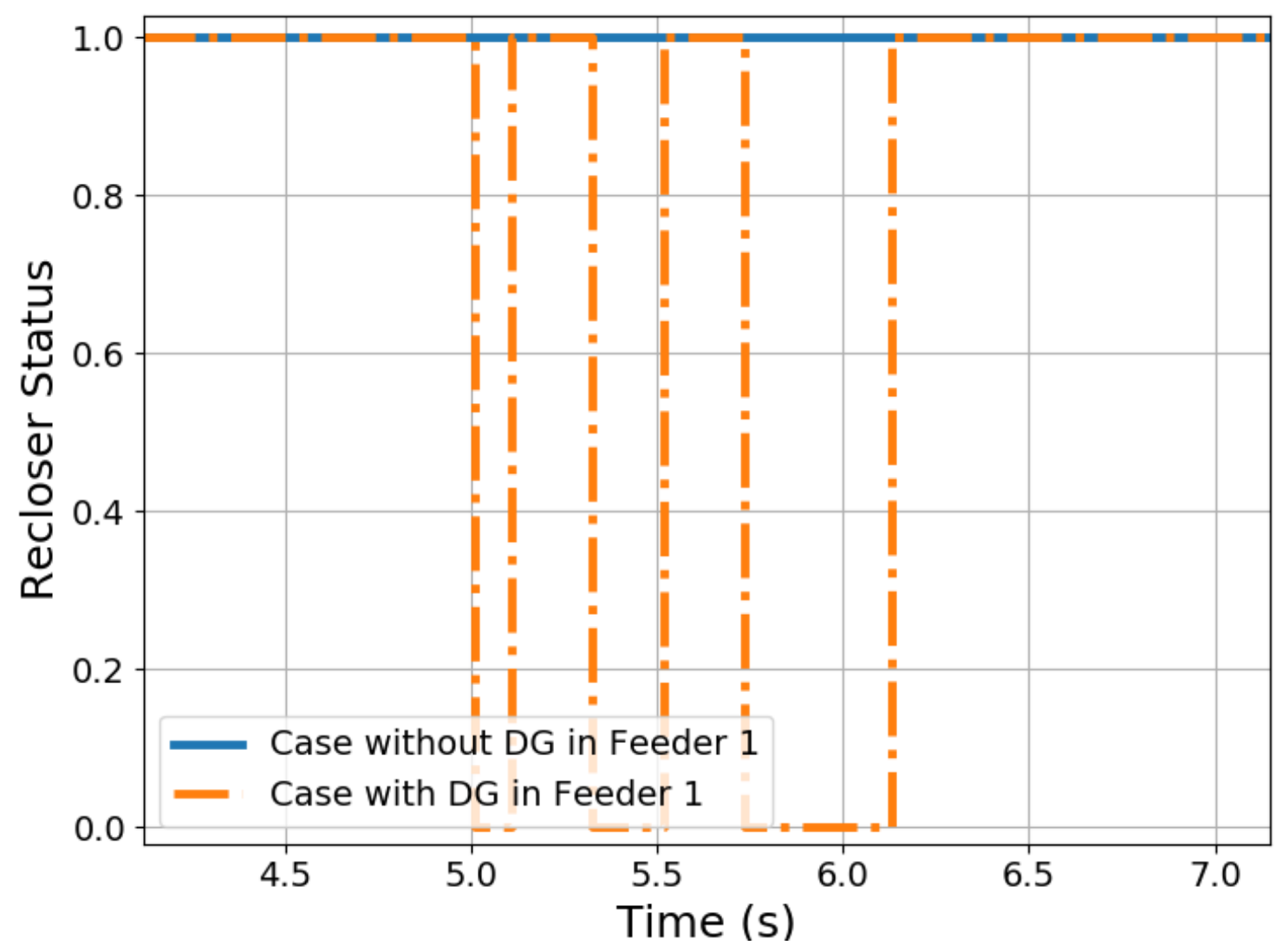

Figure 64 Feeder \#1 Recloser Status Comparison for a Case with and without DG.

\subsection{NUISANCE TRIPPING}

The fault current contribution from DGs can cause a fuse protecting a lateral that would have otherwise remained intact to blow out. When a recloser operates in a fast-operating curve, it clears the fault before the lateral fuse blows. However, when a DG is present, the sequence is such that when the recloser opens, DGs can continue to feed the fault, which leads to a fuse blowing that would not have blown. The type and location of DGs can thus compromise the fuse-saving capability intended for reclosers in an existing distribution system's protection coordination. Thus, the addition of DGs can lead to sustained outages for customers in certain laterals of a distribution system that would have had only momentary outages. 


\subsection{DESENSITIZING FEEDER HEAD RELAYS}

When the fault impedance is non-zero and DERs are contributing to the fault current, its contribution from the substation or upstream source may be reduced. The reduction in fault current will desensitize the protection relays at the source.

In this case, the feeder head protection relay does not operate in the way it is supposed to do: by interrupting and isolating a fault after its occurrence. Figure 65 illustrates that the desensitizing of a feeder head protection relay can occur when the DER unit feeds a downstream fault. In this case, the fault current supplied by the DER in Feeder 1 can reduce the amount of fault current supplied from the substation, causing the circuit breaker at the head of the feeder to avoid tripping.

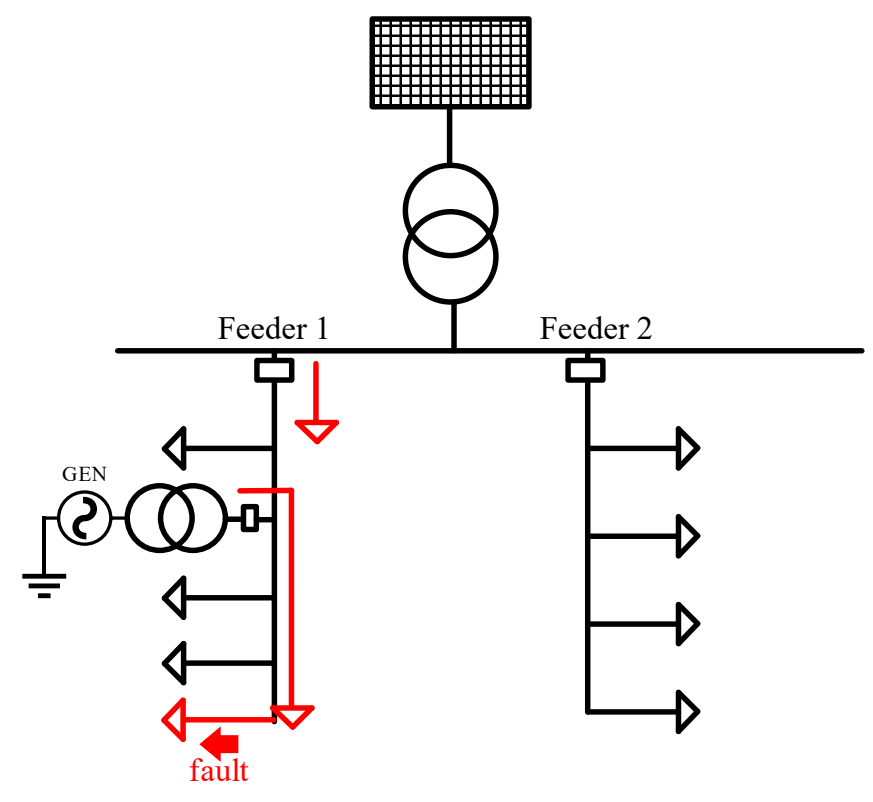

Figure 65 Desensitizing of a Feeder Head Protection Relay due to DG Integration.

The occurrence of failure to trip depends on factors like the location of the fault in the feeder, the types of DERs in the feeder (inverter-based DERs may not contribute as large a fault current as those of synchronous generator-based DERs), and the relative location of DERs with respect to fault and so on.

One of the two IEEE 34-node test feeders shown in Figure 61 is used to demonstrate the effects of the DER desensitizing feeder head's protection relays. The recloser at the head end of the feeder was operated in the fuse-saving mode, that is, as soon as the reclosers detect an increased amount of current, they open the feeder completely and allow the fault to clear itself without letting the fuse blow. 
DGs are connected at eight different nodes in the system. A fault that lasts for 0.75 second is applied at 5 seconds on node 836 of the feeder. Each of the DGs connected in the distribution feeder is rated at $200 \mathrm{~kW}$. The nominal load in the system without DGs was 2.03 MW and 0.327 MVar. The nominal current flowing into the feeder from the feeder head is 54.887 A when no DGs were connected in the feeder.

Table 11 provides information showing that the addition of DGs on the feeder has a significant impact on the fault current measured at the feeder head. As the circuit breaker at the feeder head is configured to operate based on an overcurrent scheme, the reduced amount of fault current affects its operation. Also note the increase in the first recloser's operation time in Table 11. Because of the presence of DGs on the distribution feeder, the recloser in the feeder head takes longer to operate, thus exposing the feeder to the fault for a longer period of time. This increased exposure to a fault can lead to damage of the equipment on the distribution feeder. Figure 66 shows the impact of the addition of DGs into the distribution feeder on the operation of the feeder head's recloser. It is evident that beyond a certain threshold, the rise in DGs means that it takes a longer time to operate the feeder head recloser, which exposes the feeder to the fault for a longer period of time.

Table 11 Summary of Feeder Head Fault Currents with Varying Levels of DG Penetrations.

\begin{tabular}{lcc}
\hline \multicolumn{1}{c}{ Location of DGs } & $\begin{array}{c}\text { Fault Current at } \\
\text { Feeder Head }\end{array}$ & $\begin{array}{c}\text { Feeder Head Recloser } \\
\text { First Operation Time }\end{array}$ \\
\hline & & \\
No DG & $268.558 \mathrm{~A}$ & $0.010 \mathrm{~s}$ \\
$832 \_2$ & $260.038 \mathrm{~A}$ & $0.010 \mathrm{~s}$ \\
$832 \_2,824$ & $227.901 \mathrm{~A}$ & $0.010 \mathrm{~s}$ \\
832_2, 824, 850 & $197.352 \mathrm{~A}$ & $0.010 \mathrm{~s}$ \\
832_2, 824, 850,850_1 & $172.981 \mathrm{~A}$ & $0.092 \mathrm{~s}$ \\
832_2, 824,850,850_1, 808 & $153.249 \mathrm{~A}$ & $0.106 \mathrm{~s}$ \\
$832 \_2,824,850,850 \_1,808,802$ & $138.119 \mathrm{~A}$ & $0.122 \mathrm{~s}$ \\
$832 \_2,824,850,850 \_1,808,802,806$ & $124.404 \mathrm{~A}$ & $0.144 \mathrm{~s}$ \\
$832 \_2,824,850,850 \_1,808,802,806,814$ & $107.941 \mathrm{~A}$ & $0.189 \mathrm{~s}$ \\
\hline
\end{tabular}




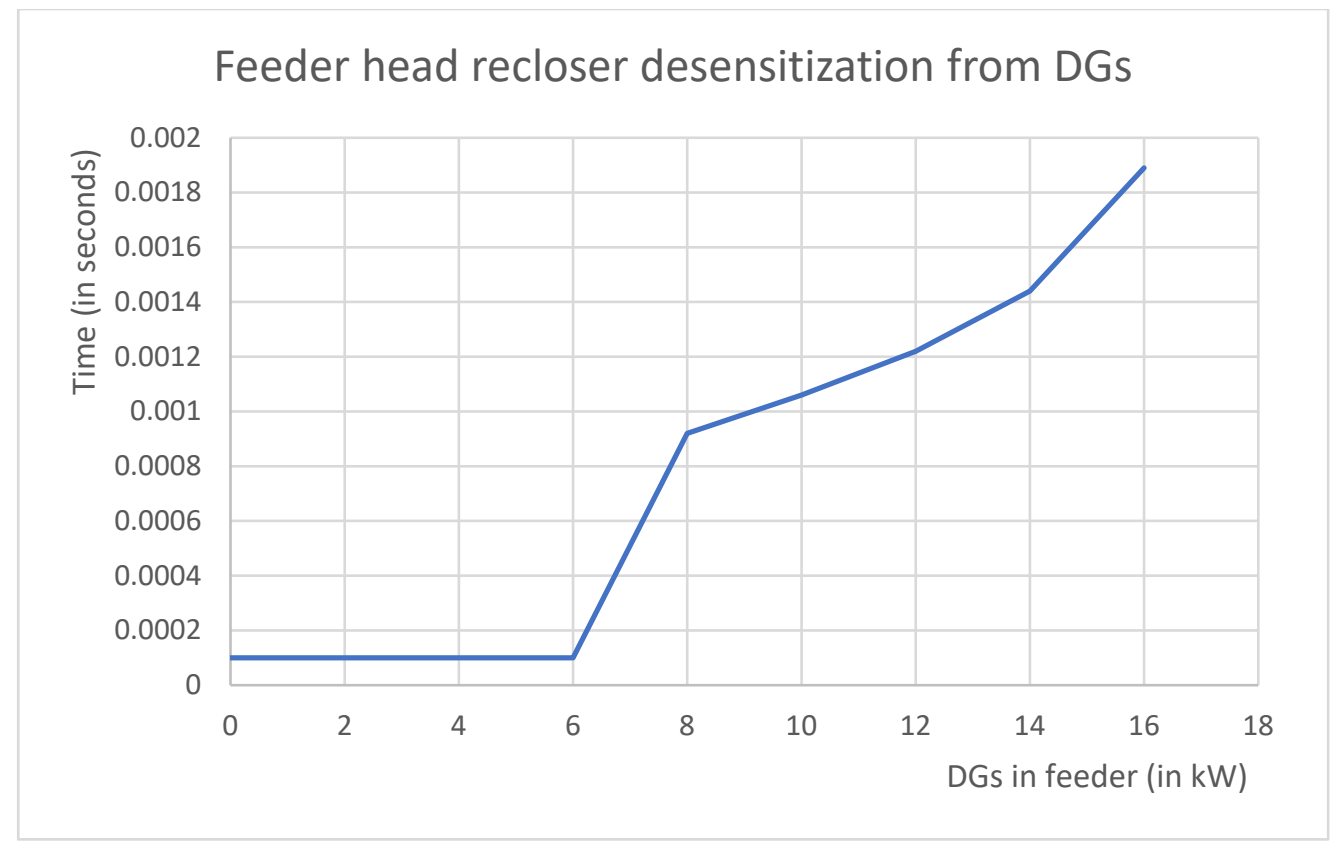

Figure 66 Impact of DGs on the Recloser's Operation Time.

\subsection{POTENTIAL SOLUTIONS FOR DISTRIBUTION SYSTEM PROTECTION}

Current distribution system protection devices suffer from inaccurate settings, because they are derived from short-circuit analysis without accounting for contributions from DERs' fault current. Moreover, these settings are manually pre-loaded into relays and therefore cannot be adjusted according to the latest DER states to ensure sensitivity and selectivity. To address these challenges, the distribution system could deploy a real-time setting update system to perform bidirectional, short-circuit analyses with the as-operated circuit connectivity and knowledge of DERs in the system [46]. Frequency of execution is governed by the communication capabilities of the distribution system. If the communication bandwidth and speed allow, an execution cycle of one minute or even less can be assumed in this discussion. The distribution system will communicate the dynamic setting update to all of the feeder protection devices through supervisory control and data acquisition (SCADA) every minute.

The lack of protection coordination between protection devices for DERs and distribution feeders can lead to nuisance fuse blowing, reclosing out of synchronism, sectionalizer miscounting, and so on. In one potential solution, local intelligence is acquired at the distribution protection device, such as the recloser, to determine whether the fault is temporary or permanent; and then communication-based local coordination is launched to integrate the sequence of actions for the protection devices for the DERs and distribution feeders. Another practical solution does not require that communication take place between the two kinds of protection devices but rather coordinates their actions by managing the DER voltage/frequency-deviationbased protection function and anti-islanding scheme. In addition, operators may be required to install additional voltage sensing relays to protection devices for the distribution feeders (such as reclosers) to facilitate proper coordination. 


\section{CONCLUSIONS}

This report discusses the various approaches that have been utilized to study the reliability impact of DERs on the BES. With the penetration of DERs on the rise, it is critical that power system operators have a high-fidelity model they can use to understand the possible impacts of DERs on the BES with a high degree of confidence. In this work, we have implemented both the aggregated and full distribution system modeling approaches and compared their performance in BES reliability studies. Even though the aggregated modeling approach provides benefits in terms of faster simulation runtime and reduced system complexity, the results obtained from the two types of models (aggregated and full) can differ considerably depending on the parameters used for these models and the nature of disturbances in the system. Given the shortcomings of the aggregated modeling approach, the full distribution system model - that includes the non-aggregated distribution system model and individual DER models - is more appropriate for studying the impact of DERs on BES stability and reliability. In this work, a T\&D combined model was developed in MATLAB/Simulink software. The T\&D combined model features the full distribution system models of the IEEE 34-node feeder and the transmission system model of the Kundur Two-Area system.

Using the T\&D combined model, we have investigated DERs' impact on BES frequency control and strategies to mitigate the impact. As a result of our findings, we propose use of a virtual-synchronous-generator-based control scheme for DERs. Case studies show that the system frequency nadir decreases following a sudden load increase when DERs without virtual inertia are integrated as compared to the base case without any DERs. This result occurs when DERs displace synchronous generators and reduce the system's total available inertia. When DERs equipped with the VSG control are present, the system frequency nadir increases following a sudden load increase event as compared to the base case without any DERs.

Furthermore, we have studied the DERs' impacts on BES net load variability and ramping, enabling us to discuss and implement mitigation schemes in the test cases. In particular, we conducted a case study based on CAISO's operational data and observed that when PV penetration increases, the net load in the BES drops during the daytime because of the surplus power supply from PVs, and then the net load ramps up at a faster rate during the evening as the sunset coincides with a period of increased load demand. In response, we implemented a BES net load smoothing algorithm that utilizes a battery energy storage system such that the BESS absorbs excess power from PVs during the daytime and discharges to serve the load during evening time. The effectiveness of the mitigation scheme in smoothing the BES net load is illustrated in a case study.

Again, using the T\&D combined model, this report evaluates various impacts of DERs on the voltage stability of the BES during normal operations. It is observed that various slopes of DERs' Volt-VAR control curves coupled with time delays can affect BES voltage regulations. A slow response coupled with steep Volt-VAR settings in DERs may cause system instability. In the meantime, when the slope of the DER Volt-VAR curve is fixed at a steeper level, time delays in the DER reactive power play a role in system stability. When no time delay is introduced, the system is able to maintain stability. When time delays are introduced, however, as soon as the 
Volt-VAR control is enabled, DERs' reactive power starts oscillating and the BES is not able to dampen the oscillations. Additional case studies we conducted suggest that interactions among multiple DERs with steep Volt-VAR settings and significant time delays may cause sustained system oscillations. Therefore, it is crucial that the DER voltage support settings be well coordinated with their response lags to avoid stability issues.

In addition to the impact on normal operations, this report explores the role that DER's reactive power support plays on distribution system and transmission system voltages and BES frequencies during abnormal conditions such as a severe transmission fault. First of all, the results of case studies establish that when the penetration of DERs without local reactive power support increases in the system, transmission system power flow oscillations (both in magnitude and duration), voltage sags, and frequency recovery all worsen. Then, we demonstrate that overall DER dynamic reactive power support helps maintain system stability compared to the case where DER does not provide any dynamic reactive power support. However, the system is sensitive to the settings of the DER reactive power support in that steep Volt-VAR curves may cause the damping of the generator speed oscillations to deteriorate, among other observed effects.

Finally, the report discusses the distribution system protection challenges brought about by high levels of DER penetration. Select case studies demonstrate that high DER penetration in a distribution system can increase fault current, cause sympathetic tripping and nuisance tripping, and desensitize feeder head protection relays. 


\section{APPENDIX A: PROCEDURE TO OBTAIN AGGREGATED DISTRIBUTION SYSTEM MODELING}

The equivalent aggregated distribution system model includes an equivalent feeder line, an aggregated load model, and an aggregated photovoltaic (PV) model (see Figure 5.). The procedure to obtain the aggregated distribution system model utilized in this study can be explained using the following steps:

\section{Step I: Load Flow}

First, a load flow analysis is performed for the distribution feeder shown in Figure 2 without the distributed PV systems. The load flow analysis was performed using MATLAB/Simulink. The goal of the load flow analysis is to estimate the equivalent system parameters for the aggregated model of the distribution feeder. The results from the load flow analysis for this case are as follows:

a) Total Active and Reactive power (PQ) load: $P=14.124 \mathrm{MW}, Q=1.74 \mathrm{Mvar}$

b) Total Z shunt load: $P=7.80 \mathrm{MW}, Q=1.74$ Mvar

c) Total Losses: $P_{\text {loss }}=0.79 \mathrm{MW}, Q_{\text {loss }}=3.45 \mathrm{Mvar}$

Therefore, from (a) and (b) above, the total load in the representative distribution feeder in Figure 2 for the operating point considered is: $P_{\text {load }}=22.72 \mathrm{MW}, Q_{\text {load }}=6.93 \mathrm{Mvar}$.

\section{Step II: Equivalent Feeder Line Parameters}

In this study, the equivalent feeder line parameters are estimated based on the overall losses in the distribution feeder as mentioned in [47]. The feeder line is represented by an equivalent $\pi$ section consisting of equivalent shunt capacitance $\left(C_{e q}\right)$, a series resistance $\left(R_{e q}\right)$, and a series inductance $\left(L_{e q}\right)$.

i) Equivalent Capacitance $\left(C_{e q}\right)$ : The reactive power generated by the shunt capacitance is proportional to the product of the square of voltage across them and the susceptance of the capacitor. The reactive power generation from the shunt capacitance of the equivalent feeder line equals the sum of all the reactive power generation from the shunt capacitance of all the lines in the distribution feeder. Under the nominal operating conditions of the system, bus voltages can be assumed to be around 1 p.u., and the shunt susceptance $\left(B_{\text {eq }}\right)$ of the equivalent feeder line can then be approximated as the sum of the shunt susceptance of all of the lines in the feeder, that is:

$$
\begin{gathered}
B_{e q}=\sum_{i=1}^{n} B_{i}=B_{\text {per unit length }} \times \text { Total length of the lines } \\
\rightarrow C_{e q}=\frac{B_{e q}}{2 \pi f} .
\end{gathered}
$$


The total length of lines can be obtained from the distribution feeder parameters in [1].

ii) Equivalent Resistance and Inductance $\left(R_{e q}\right.$ and $\left.L_{e q}\right)$ : From the load flow analysis of the distribution feeder, the active power loss $\left(P_{\text {loss }}\right)$ and reactive power loss $\left(Q_{\text {loss }}\right)$ can be used to calculate equivalent feeder resistance $\left(R_{e q}\right)$ and inductance $\left(L_{e q}\right)$.

The total current at the load bus in Figure 1 (still assuming no PV output) can be calculated as:

$$
3 V_{p h} I_{p h}^{*}=P_{\text {load }}+j Q_{\text {load }}
$$

where subscript $p h$ denotes the phase quantities, and $I_{p h}^{*}$ is the complex conjugate of $I_{p h}=I . I$ is the per-phase current flowing toward the aggregated load, $V_{p h}$ is the perphase voltage at the load bus, $P_{\text {load }}$ is the active power consumption of the equivalent load, and $Q_{\text {load }}$ is the reactive power consumption of the equivalent load obtained from the load flow analysis. Assuming $V_{p h}=V \angle 0, I_{p h}$ can be computed as:

$$
I=\frac{P_{\text {load }}-j Q_{\text {load }}}{3 V} .
$$

Assuming the node voltages throughout the distribution feeder averages to $V,{ }^{3}$ the reactive power generated by the equivalent shunt capacitance is:

$Q_{c}=3 V_{p h}^{2} B_{e q}$.

As the effective reactive power loss is the actual reactive power loss induced by the feeder lines of the equivalent feeder, the effective reactive power loss of the equivalent feeder is defined as [47]

$Q_{\text {eff loss }}=Q_{c}+Q_{\text {loss }}$.

Neglecting current through the shunt capacitances, the losses in the feeder line can be defined as:

$$
S_{\text {loss }}=3 I^{2}\left(R_{e q}+j X_{e q}\right) .
$$

Separating the real and imaginary components of $S_{\text {loss }}$ :

$$
R_{e q}=\frac{P_{l o s s}}{3 I^{2}}
$$

and $X_{e q}$ as:

${ }^{3}$ The authors acknowledge the fact that one of the issue with the equivalencing of the distribution system model with DER penetration is the considerable diversity of the terminal voltage of DERs connected in the distribution feeder. The assumption made here is solely for the simplicity of analysis. 


$$
X_{\text {eq }}=\frac{Q_{\text {eff loss }}}{3 I^{2}}=\frac{Q_{c}+Q_{\text {loss }}}{3 I^{2}} .
$$

Then, the equivalent feeder line inductance $L_{e q}$ equals to:

$$
L_{e q}=\frac{X_{e q}}{2 \pi f},
$$

where $f$ is the nominal system frequency $(60 \mathrm{~Hz}$ for the system considered).

Using the load flow results, parameters for the equivalent feeder are obtained for a single distribution feeder as shown in Table 12.

Table 12 Estimated Equivalent Feeder Parameters for a Single Distribution Feeder.

\begin{tabular}{ccc}
\hline$C_{e q}$ & $R_{e q}$ & $L_{e q}$ \\
\hline $1.21 \times 10^{-5} \mathrm{~F}$ & $3.26 \times 10^{-1} \Omega$ & $3.77 \times 10^{-3} \mathrm{H}$ \\
\hline
\end{tabular}

\section{Step III: Representation of a Distribution System with Multiple Distribution Feeders}

Depending on the size of the PV penetration desired in the bulk electric system (BES), the number of distribution feeders in the distribution system is scaled up, and the lump load in the transmission bus is proportionally reduced. The feeder impedances are also proportionally scaled assuming the losses increase linearly in the distribution system.

The parallel representation of " $\mathrm{n}$ " number of aggregated distribution feeders at a transmission bus is shown in Figure A-1 (a). The following assumptions are made for the aggregated model:

- All of the distribution feeders are identical. Therefore, the output of all of the PVD1 models are identical in phase and magnitude, all of the loads have the same characteristics, and the feeder line parameters are identical.

- The net equivalent load in the distribution system is equal to " $n$ " times the load in the representative distribution feeder.

- The net loss in the equivalent distribution system impedance is equal to " $n$ " times the total losses in the representative distribution feeder. 


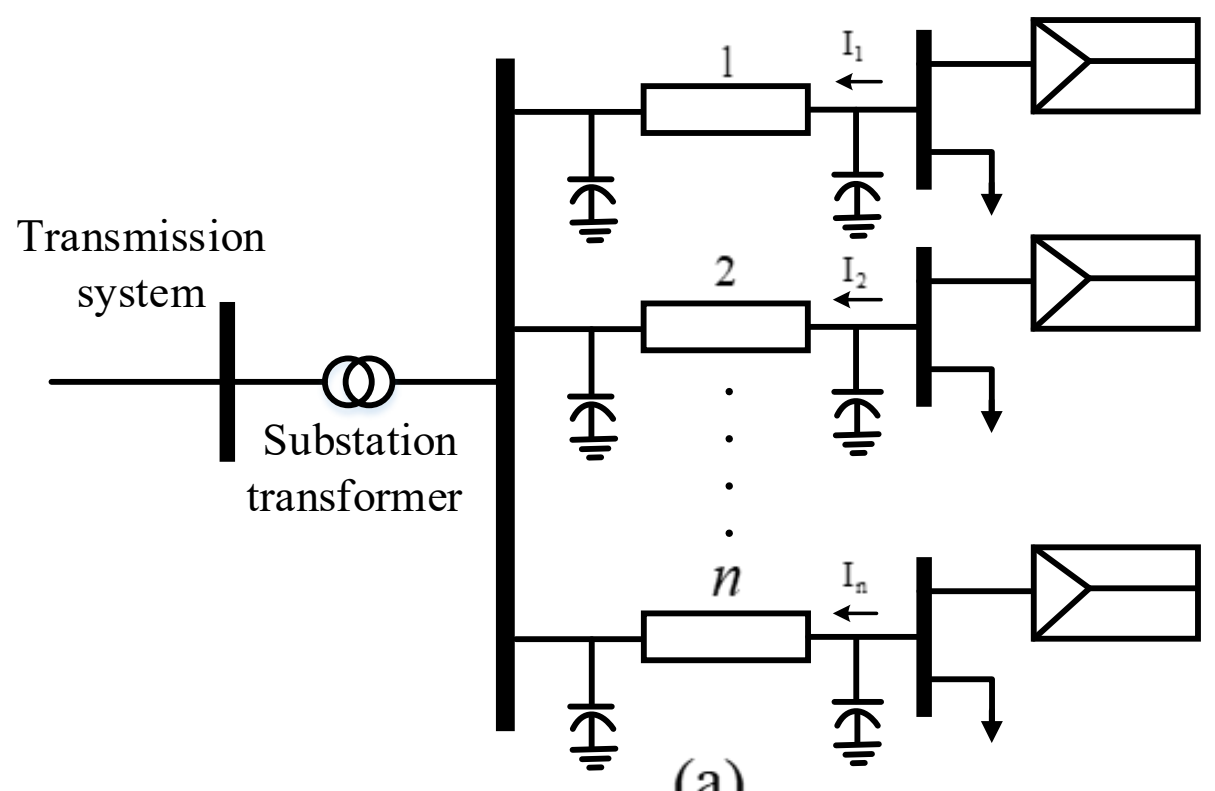

(a)

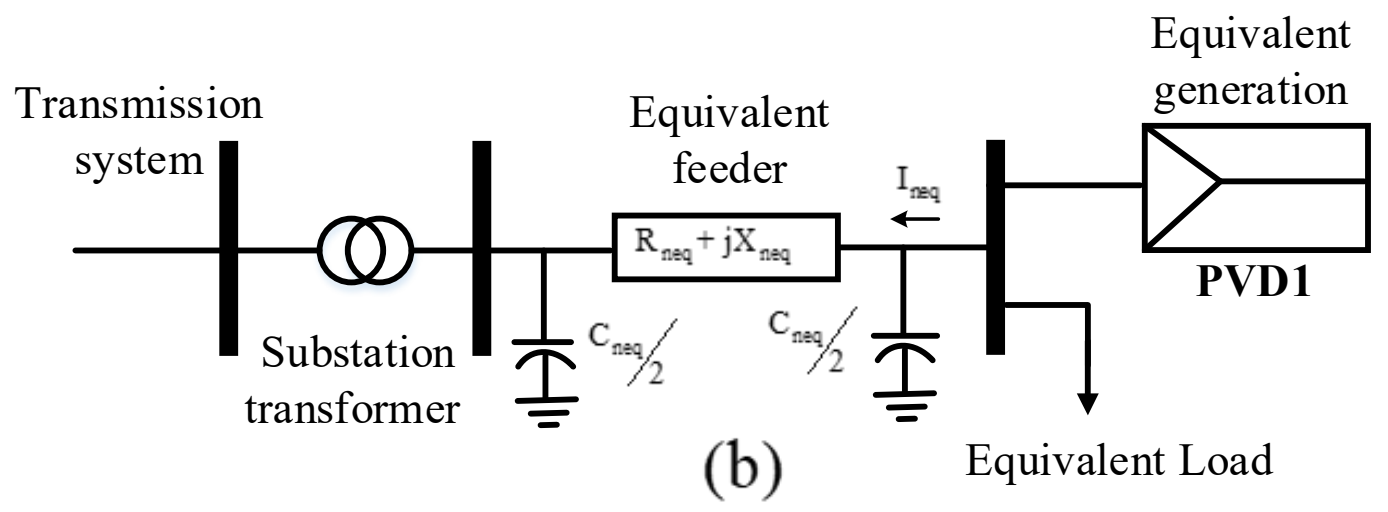

Figure A-1 (a) Equivalent Distribution System with Individual Aggregated Distribution Feeders; and (b) Equivalent Aggregated Distribution System.

Under this assumption, from Figure A-1 (a), we can write:

$$
I_{1}=I_{2}=\cdots=I_{n}=I
$$

Therefore, the total current in the equivalent representation of distribution system in Figure A-1 (b) is given by:

$$
I_{n e q}=I_{2}+I_{2}+\cdots+I_{n}=n . I .
$$

The power loss in each individual feeder line can be expressed as:

$$
\begin{aligned}
& S_{l o s s 1}=3 I_{1}^{2}\left(R_{e q 1}+j X_{e q 1}\right)=3 I^{2}\left(R_{e q}+j X_{e q}\right) \\
& S_{l o s s 2}=3 I_{2}^{2}\left(R_{e q 2}+j X_{e q 2}\right)=3 I^{2}\left(R_{e q}+j X_{e q}\right)
\end{aligned}
$$




$$
S_{\text {lossn }}=3 I_{n}^{2}\left(R_{\text {eqn }}+j X_{\text {eqn }}\right)=3 I^{2}\left(R_{e q}+j X_{\text {eq }}\right) \text {, }
$$

where $S_{\text {lossi }}$ and $\left(R_{\text {eqi }}+j X_{\text {eqi }}\right)$ are the power loss and the line parameters of the $i^{\text {th }}$ feeder, respectively. The total power loss of the equivalent representation of a distribution system in Figure A-1 (b) is given by:

$$
S_{\text {loss } \_t o t}=3 I_{\text {neq }}^{2}\left(R_{\text {neq }}+j X_{\text {neq }}\right)=3 n^{2} I^{2}\left(R_{\text {neq }}+j X_{n e q}\right) .
$$

By equating total feeder loss to the sum of the individual feeder loss:

$$
\begin{gathered}
S_{\text {loss_tot }}=S_{\text {loss } 1}+S_{\text {loss } 2}+\cdots+S_{\text {lossn }} \\
3 n^{2} I^{2}\left(R_{\text {neq }}+j X_{\text {neq }}\right)=3 n I^{2}\left(R_{e q}+j X_{\text {eq }}\right) .
\end{gathered}
$$

The equivalent line parameters of the equivalent distribution system can be calculated as:

$$
R_{n e q}+j X_{n e q}=\frac{\left(R_{e q}+j X_{e q}\right)}{n} .
$$

As discussed earlier, the reactive power generation from the shunt capacitance of the equivalent feeder line equals the sum of all of the reactive power generation from the shunt capacitance of all of the individual line feeders:

$$
\begin{gathered}
Q_{c_{t o t}}=Q_{c 1}+Q_{c 2}+\cdots+Q_{c n} \\
3 V_{e q}^{2} B_{n e q}=3 V_{1}^{2} B_{e q 1}+3 V_{2}^{2} B_{e q 2}+\cdots+3 V_{n}^{2} B_{e q n}
\end{gathered}
$$

Considering the assumptions made thus far, we also deduce that

$$
\begin{aligned}
V_{1}=V_{2} & =\cdots=V_{n}=V \\
B_{e q 1}=B_{e q 2} & =\cdots=B_{\text {eqn }}=B_{\text {eq }},
\end{aligned}
$$

and:

$$
V_{e q}=V
$$

Then:

$$
3 V^{2} B_{\text {neq }}=3 V^{2} B_{e q}+3 V^{2} B_{e q}+\cdots+3 V^{2} B_{e q}=3 n V^{2} B_{e q}
$$

Therefore, the equivalent susceptance for the total of " $n$ " equivalent feeders is given by:

$$
B_{n e q}=n B_{e q} .
$$


The equivalent shunt capacitance of the distribution system is then calculated as:

$$
\begin{gathered}
C_{n e q}={ }^{B_{n e q} / 2 \pi f}={ }^{n B_{e q}} / 2 \pi f \\
C_{n e q}=n C_{e q} .
\end{gathered}
$$

\section{Step IV: Power Output from the PVD1 Model}

To obtain the total power output from equivalent PVD1 model in the distribution system, the total PV power output at different locations in the distribution feeder are added up and multiplied by the number of distribution feeders. This is then used as the reference to the PVD1 model Such that the net equivalent generation in a distribution system is equal to $n$ times the total PV system generation in the representative distribution feeder. The typical parameters suggested in [7] are used for the PVD1 model used in the case study and are shown in Table 4.

Figure A-2 shows the flowchart that summarizes the process to obtain the equivalent aggregated model of a given distribution system.

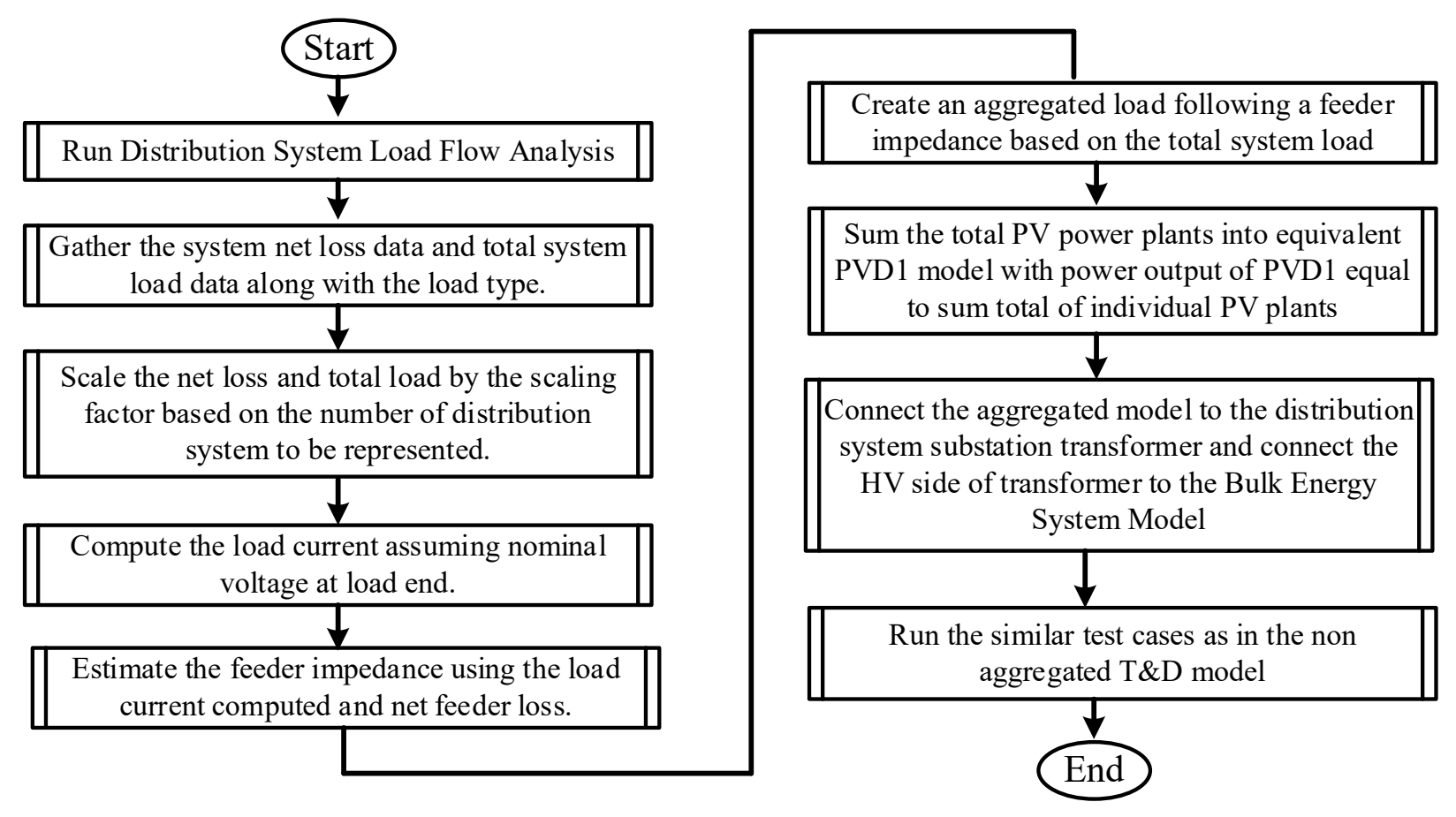

Figure A-2 Flowchart of the Process for Obtaining the Aggregated Model of a Distribution System. 


\section{APPENDIX B: OPERATING CONDITIONS FOR THE T\&D NETWORKS FOR THE CASE STUDIES (16\%, 32\%, AND $48 \%$ WITH RESPECT TO LOAD IN AREA 2)}

Table B-1 provides operating conditions for the transmission and distribution (T\&D) networks used in the case studies for Area 2 with a $16 \%$ penetration rate for distributed energy resources (DERs); Table B-2 does the same for a 32\% DER penetration rate; and Table B-3 for a $48 \%$ DER penetration rate.

Table B-1 Operating Conditions for the T\&D Networks for the Case Studies with a 16\% DER Penetration with Respect to Load in Area 2.

\begin{tabular}{|c|c|c|c|c|}
\hline \multicolumn{5}{|l|}{ Generator Data } \\
\hline & G1 & $\mathrm{G} 2$ & G3 & G4 \\
\hline Power Output (MW) & 673.2 & 486.85 & 565 & 565 \\
\hline No. of Generators & 10 & 10 & 8 & 8 \\
\hline Inertia (s) & 6.5 & 6.5 & 5.37 & 5.37 \\
\hline Automatic Generation Control & Disabled & Disabled & Disabled & Disabled \\
\hline Power System Stabilizer & Enabled & Enabled & Enabled & Enabled \\
\hline Excitation System & Enabled & Enabled & Enabled & Enabled \\
\hline \multicolumn{5}{|l|}{ Aggregated Load Data (Transmission Node) } \\
\hline Load in Area 1 & \multicolumn{4}{|c|}{967 MW, 100 MVAR (inductive) Constant Z } \\
\hline Shunt Capacitors in Area 1 & \multicolumn{4}{|c|}{387 MVAR (capacitive) Constant Z } \\
\hline Load in Area 2 Transmission Bus & \multicolumn{4}{|c|}{ 1,339 MW, 0 MVAR (inductive) Constant Z } \\
\hline Shunt Capacitors in Area 2 Transmission Bus & \multicolumn{4}{|c|}{437 MVAR (capacitive) Constant Z } \\
\hline Net Load in distribution system connected in Bus 9 & \multicolumn{4}{|c|}{ Approx. 88 MW, 86 MVAR (inductive) } \\
\hline Total DER output from DERs on Distribution System & \multicolumn{4}{|c|}{$288 \mathrm{MW}$} \\
\hline Total Distribution System Load (including losses) & \multicolumn{4}{|c|}{ 395MW, 91 MVAR } \\
\hline \multicolumn{5}{|l|}{ Load Data (Distribution Feeder) } \\
\hline $\begin{array}{l}\text { No. of Distribution Feeders Connected in Distribution } \\
\text { System }\end{array}$ & \multicolumn{4}{|l|}{18} \\
\hline Total Connected Load (including losses) & \multicolumn{4}{|c|}{ 21.94 MW and 5.15 MVAR } \\
\hline Net Load in the Distribution Feeder & \multicolumn{4}{|c|}{ Approx. 4.42 MW and 4.82 MVAR } \\
\hline No. of PV System in the Distribution Feeder & \multicolumn{4}{|c|}{4} \\
\hline Rating of Individual PV System in the Distribution Feeder & \multicolumn{4}{|c|}{$4 \mathrm{MW}, 4.8 \mathrm{MVA}$ each } \\
\hline
\end{tabular}


Table B-2 Operating Conditions for the T\&D Networks for the Case Studies with a 32\% DER Penetration with Respect to Load in Area 2.

\begin{tabular}{|c|c|c|c|c|}
\hline \multicolumn{5}{|l|}{ Generator Data } \\
\hline & G1 & G2 & G3 & G4 \\
\hline Power Output (MW) & 673.2 & 486.85 & 423.75 & 423.75 \\
\hline No. of Generators & 10 & 10 & 6 & 6 \\
\hline Equivalent Inertia from Available Generators (s) & 6.5 & 6.5 & 4.027 & 4.027 \\
\hline Automatic Generation Control & Disabled & Disabled & Disabled & Disabled \\
\hline Power System Stabilizer & Enabled & Enabled & Enabled & Enabled \\
\hline Excitation System & Enabled & Enabled & Enabled & Enabled \\
\hline \multicolumn{5}{|l|}{ Aggregated Load Data (Transmission Bus) } \\
\hline Load in Area 1 & \multicolumn{4}{|c|}{967 MW, 100 MVAR (inductive) Constant $Z$ type } \\
\hline Shunt Capacitors in Area 1 & \multicolumn{4}{|c|}{387 MVAR (capacitive) Constant $Z$ type } \\
\hline Load in Area 2 Transmission Bus & \multicolumn{4}{|c|}{$951 \mathrm{MW}, 0$ MVAR (inductive) Constant $Z$ type } \\
\hline Shunt Capacitors in Area 2 Transmission Bus & \multicolumn{4}{|c|}{507 MVAR (capacitive) Constant $Z$ type } \\
\hline Net Load in Distribution System Connected in Bus 9 & \multicolumn{4}{|c|}{ Approx. $158 \mathrm{MW}, 175$ MVAR (inductive) } \\
\hline Total Output from DERs on the Distribution System & \multicolumn{4}{|c|}{$576 \mathrm{MW}$} \\
\hline Total Distribution System Load (including losses) & \multicolumn{4}{|c|}{ 785MW, 180 MVAR } \\
\hline \multicolumn{5}{|l|}{ Load Data (Distribution Feeder) } \\
\hline No. of Feeders Connected in the Distribution System & \multicolumn{4}{|l|}{36} \\
\hline Total Connected Load (including losses) & \multicolumn{4}{|c|}{ 21.94 MW and 5.15 MVAR } \\
\hline Net Load in the Distribution Feeder & \multicolumn{4}{|c|}{ Approx. 4.42 MW and 4.82 MVAR } \\
\hline No. of PV Systems in the Distribution Feeder & \multicolumn{4}{|c|}{4} \\
\hline Rating of Individual PVs in the Distribution Feeder & \multicolumn{4}{|c|}{ 4 MW, 4.8 MVA each } \\
\hline
\end{tabular}


Table B-3 Operating Conditions for the T\&D Networks for the Case Studies with a $48 \%$ Penetration with Respect to Area 2.

\begin{tabular}{|l|l|l|l|l|}
\hline Generator Data & G1 & G2 & G3 & G4 \\
\hline Power Output (MW) & 673.2 & 486.85 & 282.5 & 282.5 \\
\hline No. of Generators & 10 & 10 & 4 & 4 \\
\hline Inertia (s) & 6.5 & 6.5 & $\sim 2.68$ & $\sim 2.68$ \\
\hline Automatic Generation Control & Disabled & Disabled & Disabled & Disabled \\
\hline Power System Stabilizer & Enabled & Enabled & Enabled & Enabled \\
\hline Excitation System & Enabled & Enabled & Enabled & Enabled \\
\hline Aggregated Load Data (Transmission Node) & \multicolumn{5}{l|}{} \\
\hline Load in Area 1 & 967 MW, 100 MVAR (inductive) Constant Z type \\
\hline Shunt Capacitors in Area 1 & 387 MVAR (capacitive) Constant Z type \\
\hline Load in Area 2 Transmission Bus & 603 MW, 0 MVAR (capacitive) Constant Z type \\
\hline Shunt Capacitors in Area 2 Transmission Bus & 607 MVAR (capacitive) Constant Z type \\
\hline Net Load in the Distribution System Connected in Bus 9 & Approx. 239 MW, 260.28 MVAR (inductive) \\
\hline Total DERs Output from DERs on the Distribution System & 864 MW \\
\hline Total Distribution System Load (including losses) & 1184.66 MW, 278.1 MVAR \\
\hline Load Data (Distribution Feeder) & \multicolumn{5}{l}{} \\
\hline No. of Feeders Connected in the Distribution System & 54 \\
\hline Total Connected Load (including losses) & 21.94 MW and 5.15 MVAR \\
\hline Net Load in the Distribution Feeder & Approx. 4.42 MW and 4.82 MVAR \\
\hline No. of PV Systems in the Distribution Feeder & 4 \\
\hline Rating of Individual PV System in the Distribution Feeder & 4 MW, 4.8 MVA each \\
\hline
\end{tabular}


This page intentionally blank. 


\section{REFERENCES}

[1]. Kang, N., R. Singh, J. Reilly, and N. Segal, "Impact of Distributed Energy Resources on the Bulk Electric System," report [for Office of Electricity, US Department of Energy], Argonne National Laboratory, 2017.

[2]. Kundur, P., N.J. Balu, and M.G. Lauby, Power System Stability and Control, EPRI Power System Engineering Series, McGraw-Hill, 1994.

[3]. "IEEE 34 Node Test Feeder," http://ewh.ieee.org/soc/pes/dsacom/testfeeders/index.html, accessed June 23, 2017.

[4]. IEEE (Institute of Electrical and Electronics Engineers), "IEEE Standard for Interconnecting Distributed Resources with Electric Power Systems," IEEE Standard 1547a-2014, May 2014.

[5]. Blaabjerg, F., et al. "Overview of Control and Grid Synchronization for Distributed Power Generation Systems," IEEE Transactions on Industrial Electronics, vol. 53, no. 5, pp. 1398-1409, 2006.

[6]. WECC Composite Load Model Specifications 01-27-2015. [Online] Available at: https://www.wecc.biz/Reliability/WECC_Approved_CMPLDW_Model_Spec.pdf, accessed February 17, 2020.

[7]. WECC Renewable Energy Modeling Task. Force, "WECC Solar Plant Dynamic Modeling Guidelines," 2014.

[8]. Pogaku, N., M. Prodanovic, and T. Green, "Modeling, Analysis and Testing of Autonomous Operation of an Inverter-Based Microgrid," IEEE Transactions on Power Electronics, vol. 22, no. 2, pp. 613-625, Mar. 2015.

[9]. Majumder, R., B. Chaudhuri, A. Ghosh, R. Majumder, G. Ledwich, and F. Zare, "Improvement of Stability and Load Sharing in an Autonomous Microgrid Using Supplementary Droop Control Loop," IEEE Transactions on Power Systems, vol. 25, no. 2, pp. 796-808, 2010.

[10]. Zhang, F., M. Hong, and H. Zhao, "Operation of Networked Microgrids in a Distribution System," CSEE Journal of Power and Energy Systems, vol. 1, no. 4, pp. 12-21, 2015.

[11]. Zhao, Z., P. Yang, Y. Wang, Z. Xu, and J.M. Guerrero, "Dynamic Characteristics Analysis and Stabilization of PV-based Multiple Microgrid Clusters," IEEE Transactions on Smart Grid, early online access, 2017.

[12]. U. Tamrakar, D. Shrestha, M. Maharjan, B. Bhattarai, T. Hansen, and R. Tonkoski, "Virtual inertia: Current trends and future directions," Applied Sciences, vol. 7, no. 7, p. 654, 2017. 
[13]. H. Bevrani, T. Ise, and Y. Miura, "Virtual synchronous generators: A survey and new perspectives," International Journal of Electrical Power \& Energy Systems, vol. 54, pp. 244-254, 2014.

[14]. A. Vassilakis, P. Kotsampopoulos, N. Hatziargyriou, and V. Karapanos, "A battery energy storage based virtual synchronous generator," in 2013 IREP Symposium Bulk Power System Dynamics and Control-IX Optimization, Security and Control of the Emerging Power Grid. IEEE, 2013, pp. 1-6.

[15]. Q.-C. Zhong and G. Weiss, "Synchronverters: Inverters that mimic synchronous generators," IEEE Transactions on industrial electronics, vol. 58, no. 4, pp. 1259-1267, 2010.

[16]. Q.-C. Zhong, "Virtual synchronous machines: A unified interface for grid integration," IEEE Power Electronics Magazine, vol. 3, no. 4, pp. 18-27, 2016.

[17]. K. Sakimoto, Y. Miura, and T. Ise, "Stabilization of a power system with a distributed generator by a virtual synchronous generator function," in 8th International Conference on Power Electronics-ECCE Asia. IEEE, 2011, pp. 1498-1505.

[18]. J. Liu, Y. Miura, H. Bevrani, and T. Ise, "Enhanced virtual synchronous generator control for parallel inverters in microgrids," IEEE Transactions on Smart Grid, vol. 8, no. 5, pp. 2268-2277, 2016.

[19]. J. Liu, Y. Miura, and T. Ise, "Comparison of dynamic characteristics between virtual synchronous generator and droop control in inverterbased distributed generators," IEEE Transactions on Power Electronics, vol. 31, no. 5, pp. 3600-3611, 2015.

[20]. J. Alipoor, Y. Miura, and T. Ise, "Stability assessment and optimization methods for microgrid with multiple vsg units," IEEE Transactions on Smart Grid, vol. 9, no. 2, pp. 1462-1471, 2016.

[21]. H. Wu, X. Ruan, D. Yang, X. Chen, W. Zhao, Z. Lv, and Q.-C. Zhong, "Small-signal modeling and parameters design for virtual synchronous generators," IEEE Transactions on Industrial Electronics, vol. 63, no. 7, pp. 4292-4303, 2016.

[22]. W. Wu, Y. Chen, A. Luo, L. Zhou, X. Zhou, L. Yang, Y. Dong, and J. M. Guerrero, “A virtual inertia control strategy for dc microgrids analogized with virtual synchronous machines," IEEE Transactions on Industrial Electronics, vol. 64, no. 7, pp. 6005-6016, 2016.

[23]. C. Andalib-Bin-Karim, X. Liang, and H. Zhang, "Fuzzy-secondarycontroller-based virtual synchronous generator control scheme for interfacing inverters of renewable distributed generation in microgrids," IEEE Transactions on Industry Applications, vol. 54, no. 2, pp. 1047-1061, 2018. 
[24]. K. Shi, W. Song, P. Xu, R. Liu, Z. Fang, and Y. Ji, "Low-voltage ridethrough control strategy for a virtual synchronous generator based on smooth switching," IEEE Access, vol. 6, pp. 2703-2711, 2017.

[25]. T. Zheng, L. Chen, Y. Guo, and S. Mei, "Comprehensive control strategy of virtual synchronous generator under unbalanced voltage conditions," IET Generation, Transmission \& Distribution, vol. 12, no. 7, pp. 1621-1630, 2017.

[26]. H. Zhao, Q. Yang, and H. Zeng, "Multi-loop virtual synchronous generator control of inverter-based dgs under microgrid dynamics," IET Generation, Transmission \& Distribution, vol. 11, no. 3, pp. 795-803, 2017.

[27]. J. Rocabert, A. Luna, F. Blaabjerg, and P. Rodriguez, "Control of power converters in ac microgrids," IEEE transactions on power electronics, vol. 27, no. 11, pp. 4734-4749, 2012.

[28]. P. Kundur, N. J. Balu, and M. G. Lauby, Power system stability and control. McGraw-hill New York, 1994, vol. 7.

[29]. W. H. Kersting, "Radial distribution test feeders," IEEE Transactions on Power Systems, vol. 6, no. 3, pp. 975-985, 1991.

[30]. J. Alipoor, Y. Miura, and T. Ise, "Distributed generation grid integration using virtual synchronous generator with adoptive virtual inertia," in 2013 IEEE Energy Conversion Congress and Exposition. IEEE, 2013, pp. 4546-4552.

[31]. T. Shintai, Y. Miura, and T. Ise, "Oscillation damping of a distributed generator using a virtual synchronous generator," IEEE transactions on power delivery, vol. 29, no. 2, pp. 668-676, 2014.

[32]. A. Fathi, Q. Shafiee, and H. Bevrani, "Robust frequency control of microgrids using an extended virtual synchronous generator," IEEE Transactions on Power Systems, vol. 33, no. 6, pp. 6289-6297, 2018.

[33]. K. Sakimoto, Y. Miura, and T. Ise, "Stabilization of a power system including inverter type distributed generators by the virtual synchronous generator," IEEJ Transactions on Power and Energy, vol. 132, pp. 341-349, 2012.

[34]. X. Zhang, D.-b. ZHU, and H.-z. XU, "Review of virtual synchronous generator technology in distributed generation," Journal of Power Supply, vol. 3, pp. 1-6, 2012.

[35]. Y. Du, J. M. Guerrero, L. Chang, J. Su, and M. Mao, "Modeling, analysis, and design of a frequency-droop-based virtual synchronous generator for microgrid applications," in 2013 IEEE ECCE Asia Downunder. IEEE, 2013, pp. 643-649.

[36]. Z. Zeng, W. Shao, L. Ran, Z. Lyu, and R. Li, "Mathematical model and strategic energy storage selection of virtual synchronous generators," Automation of Electric Power Systems, vol. 39, no. 13, pp. 22-31, 2015. 
[37]. Y. Hirase, K. Sugimoto, K. Sakimoto, and T. Ise, “Analysis of resonance in microgrids and effects of system frequency stabilization using a virtual synchronous generator," IEEE Journal of Emerging and Selected Topics in Power Electronics, vol. 4, no. 4, pp. 1287$1298,2016$.

[38]. C. Li, J. Xu, and C. Zhao, “A coherency-based equivalence method for mmc inverters using virtual synchronous generator control," IEEE Transactions on Power Delivery, vol. 31, no. 3, pp. 1369-1378, 2015.

[39]. Vlahoplus, C., G. Litra, P. Quinlan, and C. Becker, Revisiting the California Duck Curve: An Exploration of Its Existence, Impact, and Migration Potential (technical report), Scottmadden, Inc., Aug. 2016.

[40]. CAISO (California ISO), Reliability Requirements. Available at: http://www.caiso.com/planning/Pages/ReliabilityRequirements/Default.aspx\#Historical, accessed February 17, 2020.

[41]. CAISO, Supply and Renewables. Available at: http://www.caiso.com/TodaysOutlook/Pages/supply.aspx, accessed February 17, 2020.

[42]. Chakraborty, S., A. Hoke, and B. Lundstrom. "Evaluation of Multiple Inverter Volt-VAR Control Interactions with Realistic Grid Impedances," Power \& Energy Society General Meeting, 2015 IEEE, IEEE, 2015.

[43]. Li, H., J. Smith, and M. Rylander, "Multi-Inverter Interaction with Advanced Grid Support Functions," Grid of the Future Symposium, Houston, 2014.

[44]. Seguin, R., J. Woyak, D. Costyk, J. Hambrick, and B. Mather, High-Penetration PV Integration Handbook for Distribution Engineers, United States: N. p., 2016. Web. doi:10.2172/1235905.

[45]. Stewart, E., J. MacPherson, S. Vasilic, D. Nakafuji, and T. Aukai, Analysis of HighPenetration Levels of Photovoltaics into the Distribution Grid on Oahu, Hawaii: Detailed Analysis of HECO Feeder WF1, report, May 1, 2013; Golden, Colorado.

[46]. Kang, N., J. Wang, R. Singh, and X. Lu, Interconnection, Integration, and Interactive Impact Analysis of Microgrids and Distribution Systems, United States: N. p., 2017. Web. doi:10.2172/1349056.

[47]. Muljadi, E., C.P. Butterfield, A. Ellis, J. Mechenbier, J. Hochheimer, R. Young, N. Miller, R. Delmerico, R. Zavadil, and J.C. Smith, "Equivalencing the Collector System of a Large Wind Power Plant," IEEE Power Engineering Society General Meeting, pp. 1-9, 2016. 
This page intentionally blank. 



\section{Argonne $\mathbf{A}$}

\section{Energy Systems Division}

Argonne National Laboratory

9700 South Cass Avenue, Bldg. 362

Argonne, IL 60439-4832

www.anl.gov 